\title{
2003
}

Los Alamos National Laboratory Annual Illness and Injury Surveillance Report

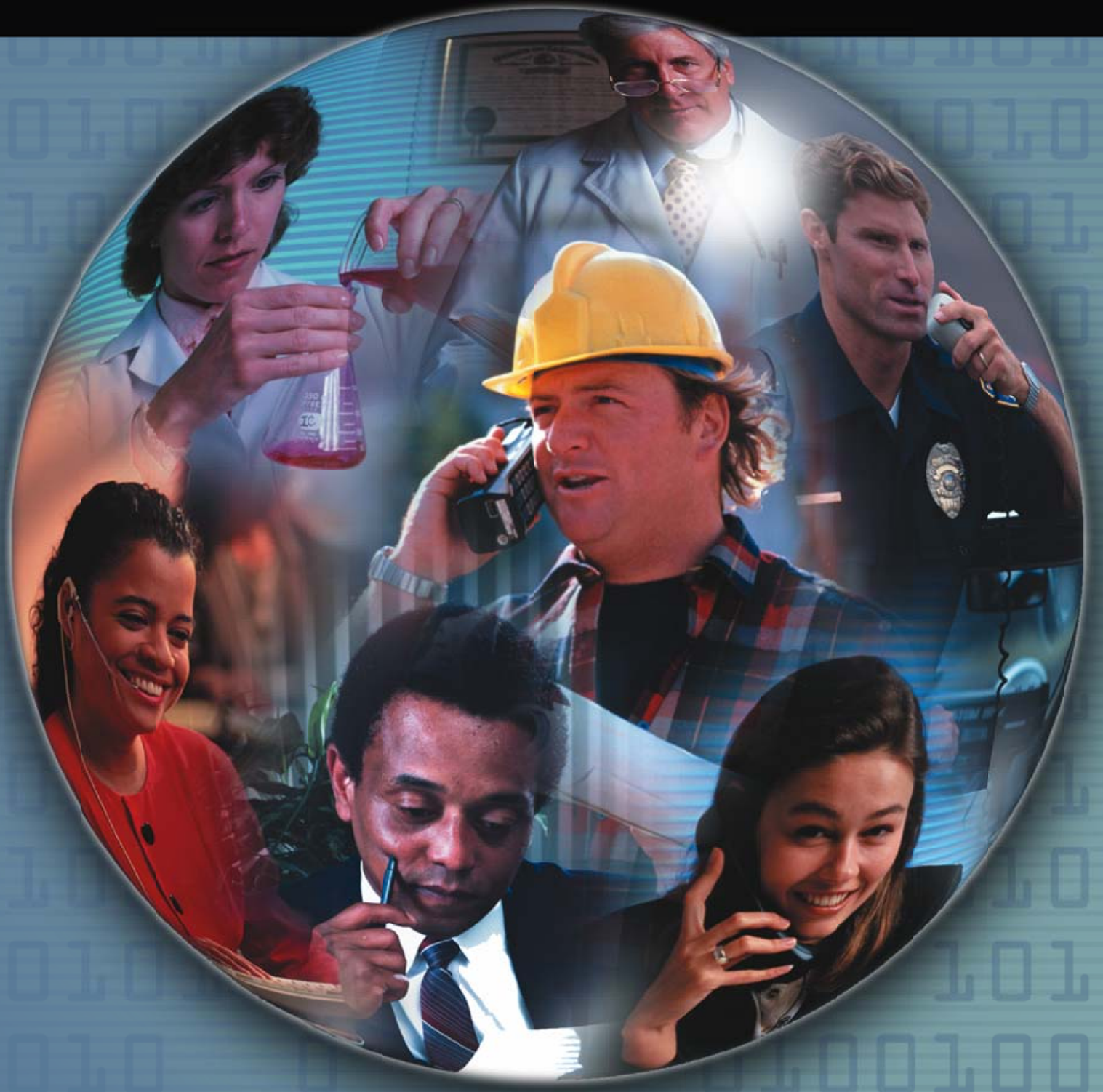




\section{Los Alamos National Laboratory 2003 Illness and Injury Surveillance Report}

Questions or comments about this report or the Illness and Injury Surveillance Program (IISP) may be directed to:

E-mail:

Dr. Cliff Strader at cliff.strader@hq.doe.gov or Dr. Bonnie Richter at bonnie.richter@hq.doe.gov

or direct letters to:

Mail Stop HS-13 / 270CC

U.S. Department of Energy

1000 Independence Avenue, S.W.

Washington, DC 20585-0270

Additional information about the Department of Energy's Office of Illness and Injury Prevention Programs, the IISP, and annual reports for DOE sites participating in this program can be found at:

http://www.hss.energy.gov/healthsafety/WSHP/epi/surv/

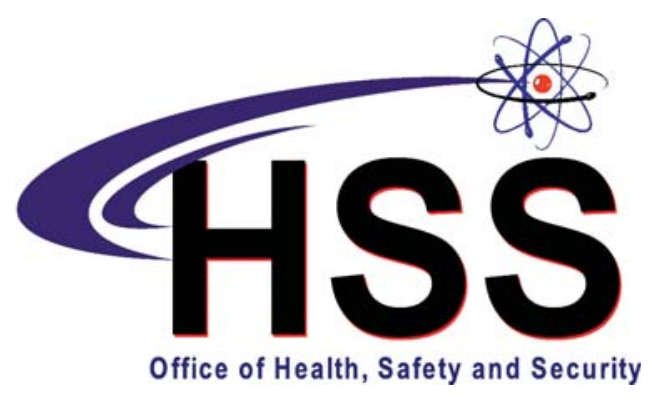


The Los Alamos National Laboratory Work Force - 2003

The Work Force by Gender and Age 1

The Work Force by Gender and Job

Category.... ..1

\section{Number and Length of Absences}

Absence Rate by Gender and Age

Number of Days Absent by

Gender and Age . .2

Absence Rate by Job Category

and Gender. 3

Average Duration of Absence by Job Category and Gender....

\section{Diagnostic Categories}

Number of Diagnoses and Lost Calendar Days by Diagnostic Category

(Categorized by ICD-9-CM) and Gender 4

Common Diagnoses Among Female

Workers in 2003

Common Diagnoses Among Male

Workers in 2003

Number of Most Frequently Reported

Diagnoses by Job Category and Gender ......7

\section{Rates of Disease Occurrence}

Rates for All Illnesses and Injuries

Combined by Job Category, Gender, and Age

Rates for Selected Diagnostic Categories by Job Category, Gender, and Age.

\section{Sentinel Health Events for Occupations (SHEOs)}

Characteristics of SHEOs by Gender.... 10

Occupational Safety and Health Administration (OSHA)-Recordable Events

OSHA-Recordable Events by Gender and Age

OSHA-Recordable Events by Job

Category and Gender

\section{Diagnostic and Accident Categories for OSHA-Recordable Events}

OSHA-Recordable Diagnoses by

Diagnostic Category and Gender

OSHA-Recordable Accidents by Type

and Gender.... 12

\section{Rates of OSHA-Recordable Events}

OSHA-Recordable Rates by Age and Job Categories Among Women, All

Diagnoses Combined

OSHA-Recordable Rates by Age and Job Categories Among Men, All

Diagnoses Combined 13

\section{Appendices}

Appendices A-W. 15 
The Los Alamos National Laboratory Work Force - 2003

Figure 1. The Work Force by Gender and Age

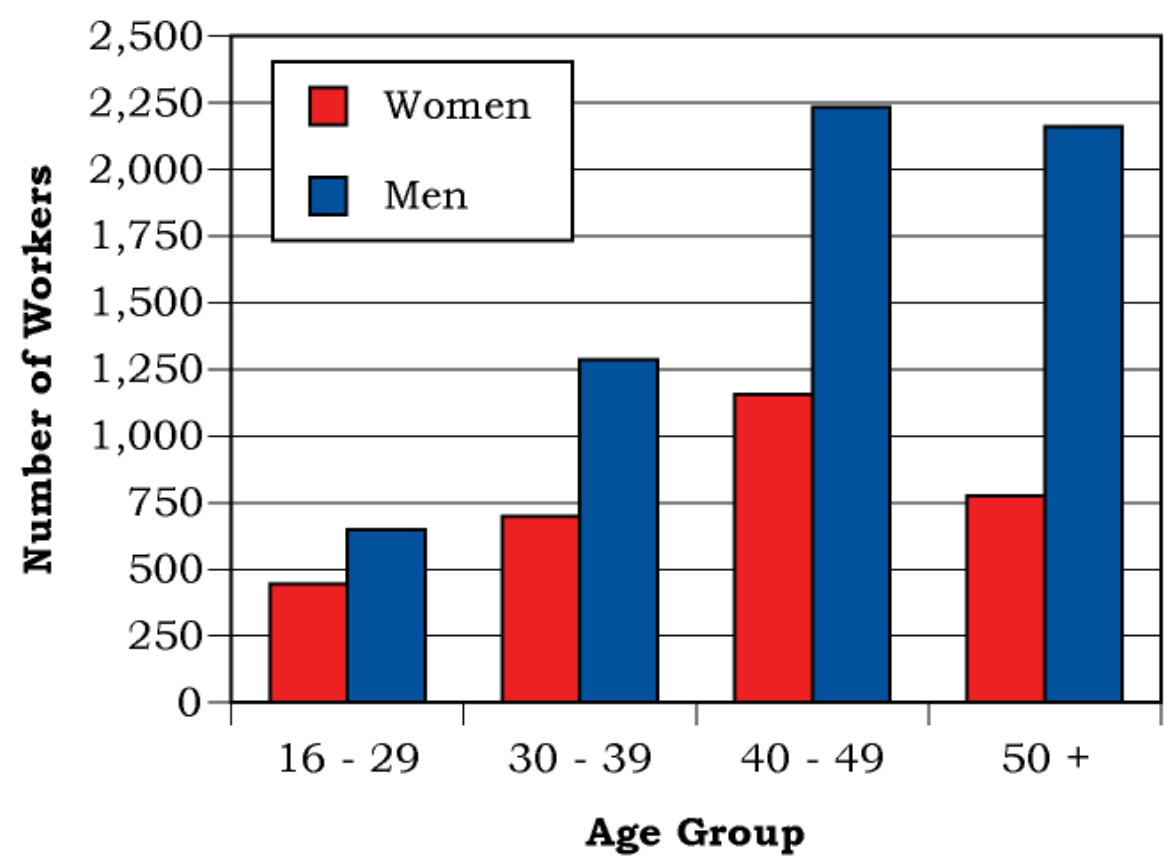

Figure 2. The Work Force by Gender and Job Category

\begin{tabular}{|l|c|c|}
\hline Job Category & Women & Men \\
\hline \multirow{2}{*}{ Professional } & 2,037 & 4,886 \\
& $66 \%$ & $77 \%$ \\
\hline \multirow{2}{*}{ Administrative Support } & 620 & 65 \\
& $20 \%$ & $1 \%$ \\
\hline \multirow{2}{*}{ Technical Support } & 412 & 1,372 \\
\hline \multirow{2}{*}{ Service } & $13 \%$ & $22 \%$ \\
\hline \multirow{2}{*}{ Security and Fire } & 4 & 7 \\
\hline \multirow{2}{*}{ Crafts } & $1 \%$ & $<1 \%$ \\
\hline \multirow{2}{*}{ Line Operators } & 0 & 0 \\
\hline \multirow{2}{*}{ Total } & $0 \%$ & $0 \%$ \\
\hline
\end{tabular}


Number and Length of Absences

Figure 3. Absence Rate by Gender and Age

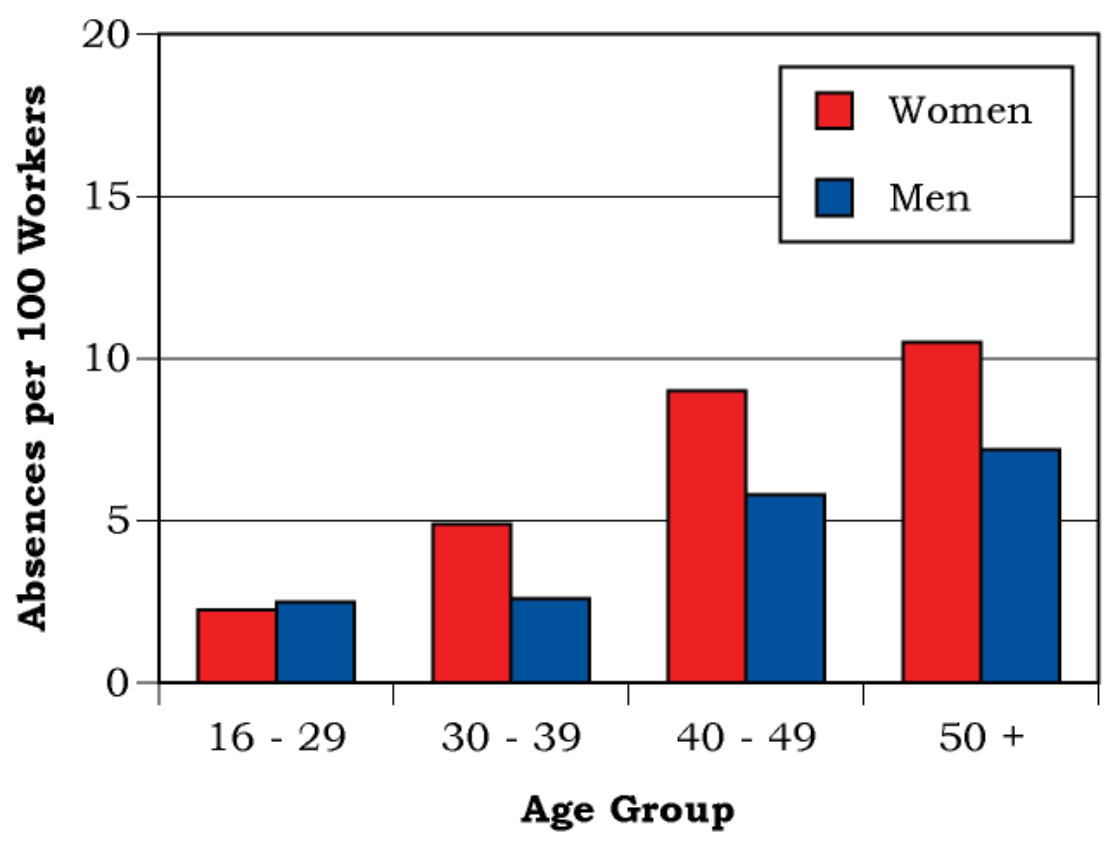

Figure 4. Number of Days Absent by Gender and Age

\begin{tabular}{|c|c|c|c|c|}
\hline \multirow{3}{*}{ Gender } & \multirow{2}{*}{ Age } & Number of & \multicolumn{2}{|c|}{ Number of Days Absent } \\
\cline { 3 - 5 } & & Absences & Total & Average \\
\hline \multirow{4}{*}{ Women } & $16-29$ & 10 & 96 & 10 \\
\cline { 3 - 5 } & $30-39$ & 34 & 584 & 17 \\
\cline { 2 - 5 } & $40-49$ & 104 & 2,468 & 24 \\
\cline { 2 - 5 } & $50+$ & 81 & 2,091 & 26 \\
\cline { 2 - 5 } & Total & 229 & 5,239 & 23 \\
\hline \multirow{4}{*}{ Men } & $16-29$ & 16 & 150 & 9 \\
\cline { 2 - 5 } & $30-39$ & 34 & 680 & 20 \\
\cline { 2 - 5 } & $40-49$ & 129 & 4,197 & 33 \\
\cline { 2 - 5 } & $50+$ & 156 & 4,400 & 28 \\
\cline { 2 - 5 } & Total & 335 & 9,427 & 28 \\
\hline
\end{tabular}


Figure 5. Absence Rate by Job Category and Gender

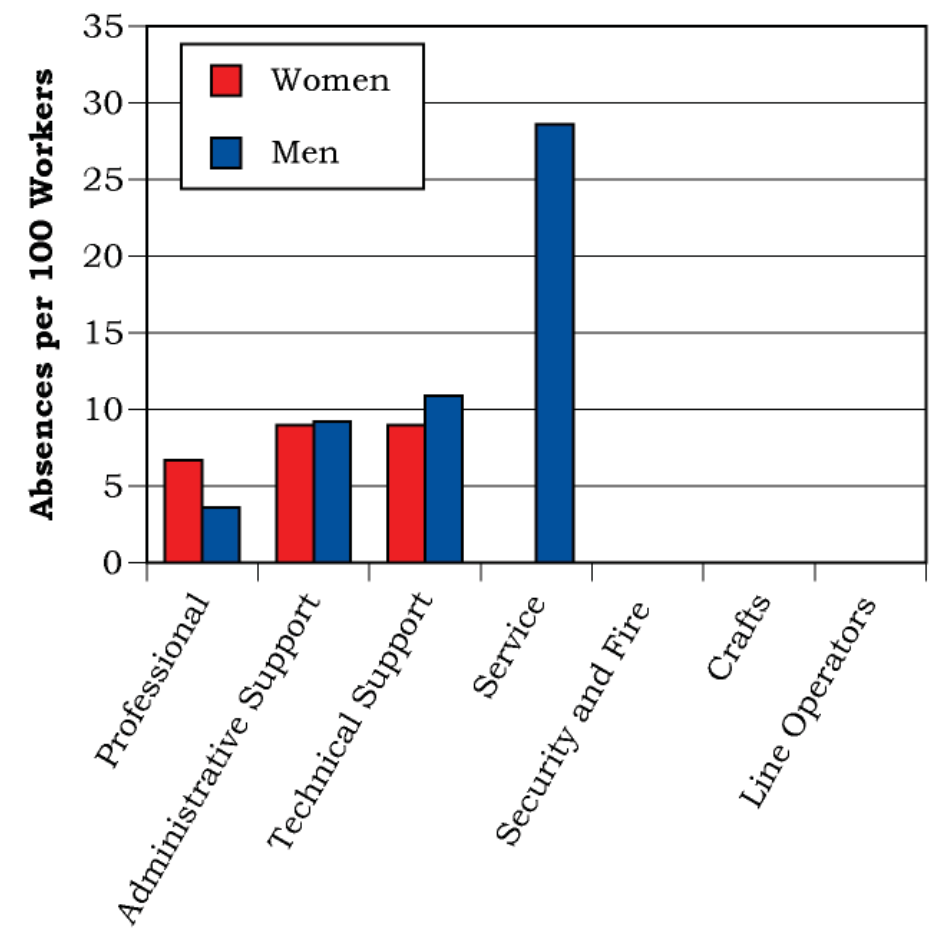

Job Category

Figure 6. Average Duration of Absence by Job Category and Gender

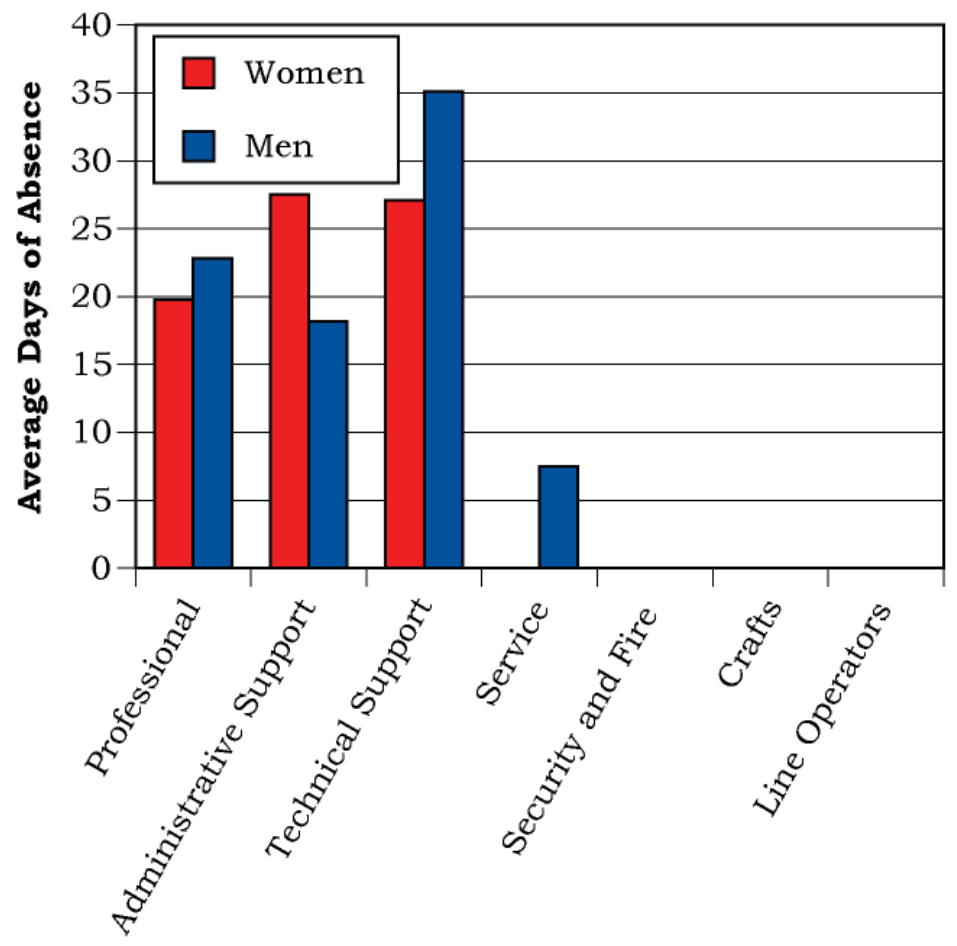

Job Category 


\section{Diagnostic Categories}

\section{Figure 7. Number of Diagnoses and Lost Calendar Days by Diagnostic Category (Categorized by ICD-9-CM) and Gender}

\begin{tabular}{|c|c|c|c|c|}
\hline \multirow[b]{2}{*}{ Diagnostic Category } & \multicolumn{2}{|c|}{ Women } & \multicolumn{2}{|c|}{ Men } \\
\hline & $\begin{array}{l}\text { Number of } \\
\text { Diagnoses }\end{array}$ & $\begin{array}{c}\text { Number } \\
\text { of Lost } \\
\text { Calendar } \\
\text { Days }\end{array}$ & $\begin{array}{l}\text { Number of } \\
\text { Diagnoses }\end{array}$ & $\begin{array}{c}\text { Number } \\
\text { of Lost } \\
\text { Calendar } \\
\text { Days }\end{array}$ \\
\hline Benign Growths & 3 & 71 & 3 & 431 \\
\hline Blood & 1 & 16 & 0 & 0 \\
\hline Cancer & 9 & 284 & 7 & 1,290 \\
\hline Digestive & 30 & 679 & 57 & 765 \\
\hline Endocrine/Metabolic & 6 & 104 & 6 & 73 \\
\hline Existing Birth Condition & 0 & 0 & 0 & 0 \\
\hline Genitourinary & 34 & 1,024 & 8 & 222 \\
\hline Heart/Circulatory & 6 & 361 & 32 & 1,532 \\
\hline Infections/Parasites & 11 & 109 & 16 & 180 \\
\hline Injury & 21 & 340 & 54 & 809 \\
\hline Miscarriage & 1 & 20 & NA & NA \\
\hline Musculoskeletal & 60 & 1,300 & 64 & 1,490 \\
\hline Nervous System & 14 & 202 & 12 & 169 \\
\hline Psychological & 12 & 281 & 11 & 1,284 \\
\hline Respiratory & 47 & 485 & 105 & 1,234 \\
\hline Skin & 0 & 0 & 3 & 78 \\
\hline Unspecified Symptoms & 15 & 228 & 23 & 354 \\
\hline
\end{tabular}

Note: Lost calendar days for each absence are counted more than once when multiple diagnoses occur in different diagnostic categories for the same absence. 
Figure 8. Common Diagnoses Among Female Workers in 2003

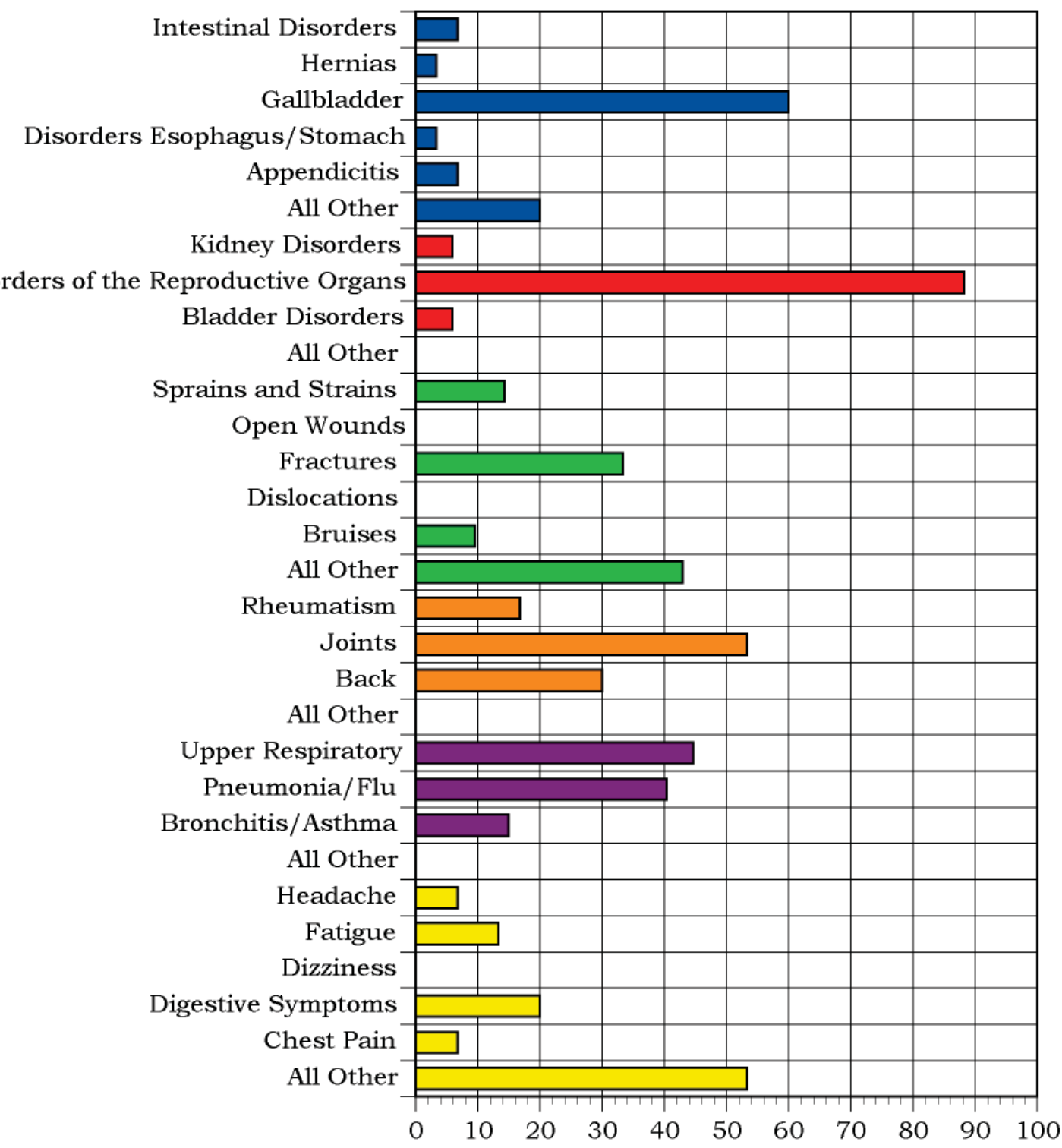

Percent Distribution of Diagnoses Within Diagnostic Category

Digestive, 30 Diagnoses

Genitourinary, 34 Diagnoses

Injury, 21 Diagnoses
Musculoskeletal, 60 Diagnoses

Respiratory, 47 Diagnoses

Unspecified Symptoms, 15 Diagnoses 
Figure 9. Common Diagnoses Among Male Workers in 2003

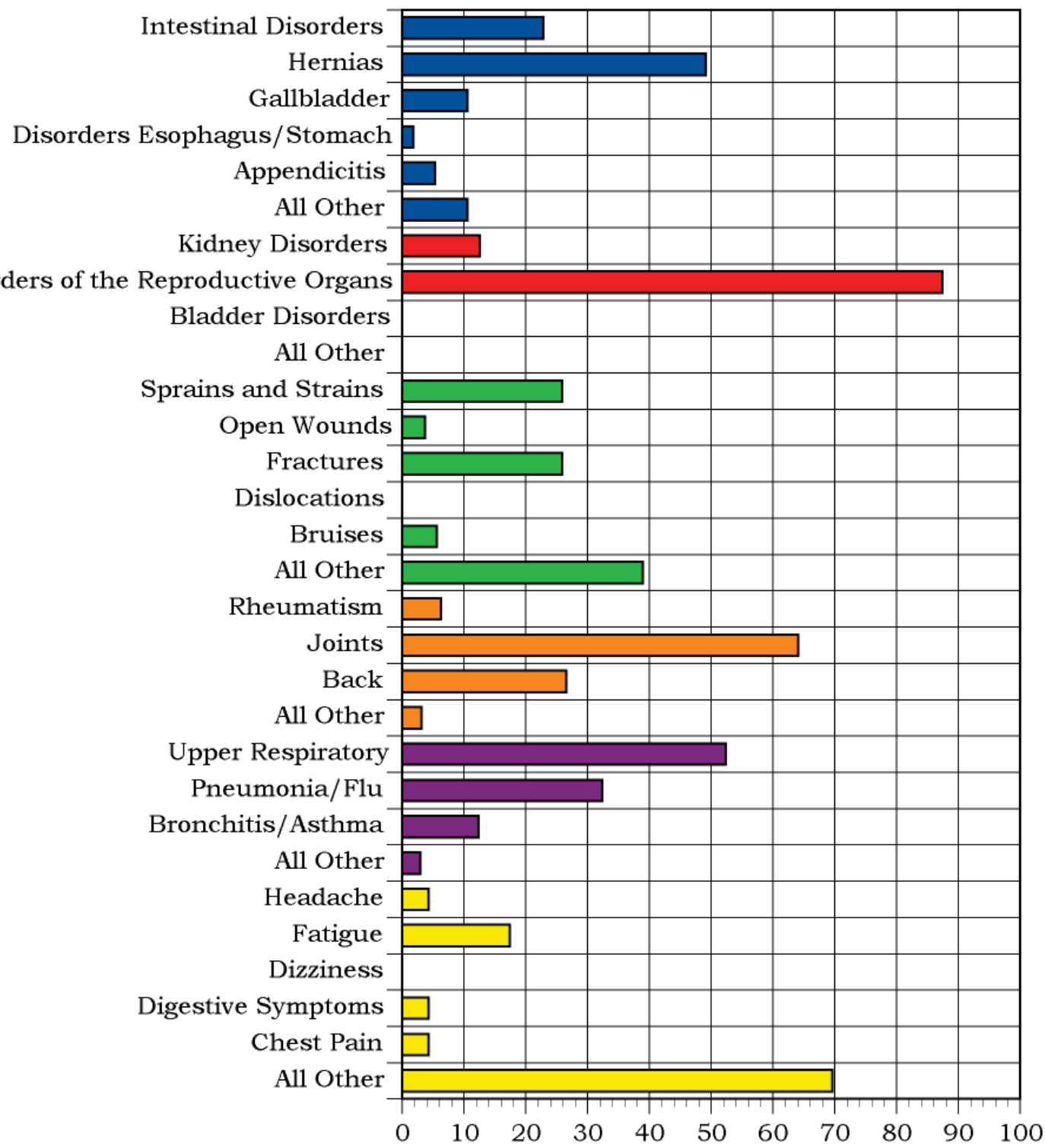

Percent Distribution of Diagnoses Within Diagnostic Category

Digestive, 57 Diagnoses

Genitourinary, 8 Diagnoses

Injury, 54 Diagnoses
Musculoskeletal, 64 Diagnoses

Respiratory, 105 Diagnoses

Unspecified Symptoms, 23 Diagnoses 
Figure 10. Number of Most Frequently Reported Diagnoses by Job Category and Gender

\begin{tabular}{|c|c|c|c|c|}
\hline Job Category & \multicolumn{2}{|l|}{ Men } & \multicolumn{2}{|c|}{ Women } \\
\hline Professional & $\begin{array}{l}\text { Respiratory } \\
\text { Digestive } \\
\text { Musculoskeletal }\end{array}$ & $\begin{array}{l}56 \\
32 \\
32 \\
\end{array}$ & $\begin{array}{l}\text { Musculoskeletal } \\
\text { Respiratory } \\
\text { Genitourinary }\end{array}$ & $\begin{array}{l}32 \\
32 \\
21\end{array}$ \\
\hline Administrative Support & $\begin{array}{l}\text { Digestive } \\
\text { Endocrine/Metabolic } \\
\text { Injury } \\
\text { Musculoskeletal } \\
\text { Respiratory }\end{array}$ & $\begin{array}{l}5 \\
1 \\
1 \\
1 \\
1\end{array}$ & $\begin{array}{l}\text { Musculoskeletal } \\
\text { Respiratory } \\
\text { Digestive } \\
\text { Genitourinary } \\
\text { Injury } \\
\end{array}$ & $\begin{array}{c}20 \\
10 \\
7 \\
7 \\
7\end{array}$ \\
\hline Technical Support & $\begin{array}{l}\text { Respiratory } \\
\text { Musculoskeletal } \\
\text { Injury }\end{array}$ & $\begin{array}{l}46 \\
31 \\
23\end{array}$ & $\begin{array}{l}\text { Musculoskeletal } \\
\text { Genitourinary } \\
\text { Injury } \\
\text { Respiratory }\end{array}$ & $\begin{array}{l}8 \\
6 \\
5 \\
5\end{array}$ \\
\hline Service & $\begin{array}{l}\text { Respiratory } \\
\text { Injury }\end{array}$ & $\begin{array}{l}2 \\
1 \\
\end{array}$ & & 0 \\
\hline Security and Fire & & 0 & & 0 \\
\hline Crafts & & 0 & & 0 \\
\hline Line Operators & & $\mathbf{0}$ & & $\mathbf{0}$ \\
\hline
\end{tabular}

\section{Rates of Disease Occurrence}

Figure 11. Rates for All Illnesses and Injuries Combined by Job Category, Gender, and Age

\begin{tabular}{|c|c|c|c|c|}
\hline \multirow{2}{*}{$\begin{array}{c}\text { All Illnesses \& } \\
\text { Injuries Combined }\end{array}$} & \multicolumn{4}{|c|}{ Rate per 1,000} \\
\hline & Job Category & Age & Men & Women \\
\hline & \multirow{2}{*}{ Professional } & $<50$ & 37 & 71 \\
\hline & & $50+$ & 56 & 107 \\
\hline & \multirow{2}{*}{ Administrative Support } & $<50$ & 122 & 95 \\
\hline & & $50+$ & 167 & 118 \\
\hline & \multirow{2}{*}{ Technical Support } & $<50$ & 94 & 84 \\
\hline & & $50+$ & 212 & 200 \\
\hline & \multirow{2}{*}{ Service } & $<50$ & 0 & 0 \\
\hline & & $50+$ & 750 & 0 \\
\hline & \multirow{2}{*}{ Security and Fire } & $<50$ & 0 & 0 \\
\hline & & $50+$ & 0 & 0 \\
\hline & \multirow{2}{*}{ Crafts } & $<50$ & 0 & 0 \\
\hline & & $50+$ & 0 & 0 \\
\hline & \multirow{2}{*}{ Line Operators } & $<50$ & 0 & 0 \\
\hline & & $50+$ & 0 & 0 \\
\hline
\end{tabular}


Figure 12. Rates for Selected Diagnostic Categories by Job Category, Gender, and Age

\begin{tabular}{|c|c|c|c|c|}
\hline \multirow{2}{*}{ Cancer } & \multicolumn{4}{|c|}{ Rate per 1,000} \\
\hline & Job Category & Age & Men & Women \\
\hline & \multirow{2}{*}{ Professional } & $<50$ & 1 & 0 \\
\hline & & $50+$ & 2 & 16 \\
\hline & \multirow{2}{*}{ Administrative Support } & $<50$ & 0 & 0 \\
\hline & & $50+$ & 0 & 0 \\
\hline & \multirow{2}{*}{ Technical Support } & $<50$ & 1 & 0 \\
\hline & & $50+$ & 0 & 13 \\
\hline & \multirow{2}{*}{ Service } & $<50$ & 0 & 0 \\
\hline & & $50+$ & 0 & 0 \\
\hline & \multirow{2}{*}{ Security and Fire } & $<50$ & 0 & 0 \\
\hline & & $50+$ & 0 & 0 \\
\hline & \multirow{2}{*}{ Crafts } & $<50$ & 0 & 0 \\
\hline & & $50+$ & 0 & 0 \\
\hline & \multirow{2}{*}{ Line Operators } & $<50$ & 0 & 0 \\
\hline & & $50+$ & 0 & 0 \\
\hline
\end{tabular}

\begin{tabular}{|c|c|c|c|c|}
\hline \multirow{2}{*}{ Heart/Circulatory } & \multicolumn{4}{|c|}{ Rate per 1,000} \\
\hline & Job Category & Age & Men & Women \\
\hline & \multirow{2}{*}{ Professional } & $<50$ & 2 & 1 \\
\hline & & $50+$ & 4 & 8 \\
\hline & \multirow{2}{*}{ Administrative Support } & $<50$ & 0 & 0 \\
\hline & & $50+$ & 0 & 0 \\
\hline & \multirow{2}{*}{ Technical Support } & $<50$ & 4 & 3 \\
\hline & & $50+$ & 37 & 0 \\
\hline & \multirow{2}{*}{ Service } & $<50$ & 0 & 0 \\
\hline & & $50+$ & 0 & 0 \\
\hline & \multirow{2}{*}{ Security and Fire } & $<50$ & 0 & 0 \\
\hline & & $50+$ & 0 & 0 \\
\hline & \multirow{2}{*}{ Crafts } & $<50$ & 0 & 0 \\
\hline & & $50+$ & 0 & 0 \\
\hline & \multirow{2}{*}{ Line Operators } & $<50$ & 0 & 0 \\
\hline & & $50+$ & 0 & 0 \\
\hline
\end{tabular}




\section{Figure 12. Rates for Selected Diagnostic Categories by Job Category, Gender, and Age (Continued)}

\begin{tabular}{|c|c|c|c|c|}
\hline \multirow{2}{*}{ Respiratory } & \multicolumn{4}{|c|}{ Rate per 1,000} \\
\hline & Job Category & Age & Men & Women \\
\hline & \multirow{2}{*}{ Professional } & $<50$ & 11 & 15 \\
\hline & & $50+$ & 12 & 17 \\
\hline & \multirow{2}{*}{ Administrative Support } & $<50$ & 0 & 20 \\
\hline & & $50+$ & 42 & 6 \\
\hline & \multirow{2}{*}{ Technical Support } & $<50$ & 20 & 15 \\
\hline & & $50+$ & 67 & 0 \\
\hline & \multirow{2}{*}{ Service } & $<50$ & 0 & 0 \\
\hline & & $50+$ & 500 & 0 \\
\hline & \multirow{2}{*}{ Security and Fire } & $<50$ & 0 & 0 \\
\hline & & $50+$ & 0 & 0 \\
\hline & \multirow{2}{*}{ Crafts } & $<50$ & 0 & 0 \\
\hline & & $50+$ & 0 & 0 \\
\hline & \multirow{2}{*}{ Line Operators } & $<50$ & 0 & 0 \\
\hline & & $50+$ & 0 & 0 \\
\hline
\end{tabular}

\begin{tabular}{|c|c|c|c|c|}
\hline \multirow{2}{*}{ Injury } & \multicolumn{4}{|c|}{ Rate per 1,000} \\
\hline & Job Category & Age & Men & Women \\
\hline & \multirow{2}{*}{ Professional } & $<50$ & 4 & 4 \\
\hline & & $50+$ & 9 & 6 \\
\hline & \multirow{2}{*}{ Administrative Support } & $<50$ & 0 & 9 \\
\hline & & $50+$ & 42 & 17 \\
\hline & \multirow{2}{*}{ Technical Support } & $<50$ & 17 & 9 \\
\hline & & $50+$ & 17 & 25 \\
\hline & \multirow{2}{*}{ Service } & $<50$ & 0 & 0 \\
\hline & & $50+$ & 250 & 0 \\
\hline & \multirow{2}{*}{ Security and Fire } & $<50$ & 0 & 0 \\
\hline & & $50+$ & 0 & 0 \\
\hline & \multirow{2}{*}{ Crafts } & $<50$ & 0 & 0 \\
\hline & & $50+$ & 0 & 0 \\
\hline & \multirow{2}{*}{ Line Operators } & $<50$ & 0 & $\mathbf{0}$ \\
\hline & & $50+$ & 0 & 0 \\
\hline
\end{tabular}




\section{Sentinel Health Events for Occupations (SHEOs)}

An occupational sentinel health event (SHEO) is a disease, disability, or death that is likely to be occupationally related. Although sentinel health events may indicate an occupational exposure, many may result from nonoccupational exposures. Sentinel health events are therefore assessed in two categories:

Definite Sentinel Health Events: Diseases that are unlikely to occur in the absence of an occupational exposure (e.g., asbestosis).

Possible Sentinel Health Events: Diseases that may be occupational but can also occur in the absence of an occupational exposure (e.g., lung cancer or carpal tunnel syndrome).

Figure 13. Characteristics of SHEOs by Gender

\begin{tabular}{|l|c|c|c|c|}
\hline \multirow{2}{*}{} & \multicolumn{2}{|c|}{$\begin{array}{c}\text { Total Number of } \\
\text { SHEO Diagnoses }\end{array}$} & \multicolumn{2}{c|}{$\begin{array}{c}\text { Total Number of } \\
\text { Days Absent }\end{array}$} \\
\cline { 2 - 5 } & Men & Women & Men & Women \\
\hline Definite & 0 & 0 & 0 & 0 \\
\hline Possible & 5 & 8 & 181 & 85 \\
\hline Total & 5 & 8 & 181 & 85 \\
\hline
\end{tabular}

Occupational Safety and Health Administration (OSHA)-Recordable Events

Figure 14. OSHA-Recordable Events by Gender and Age

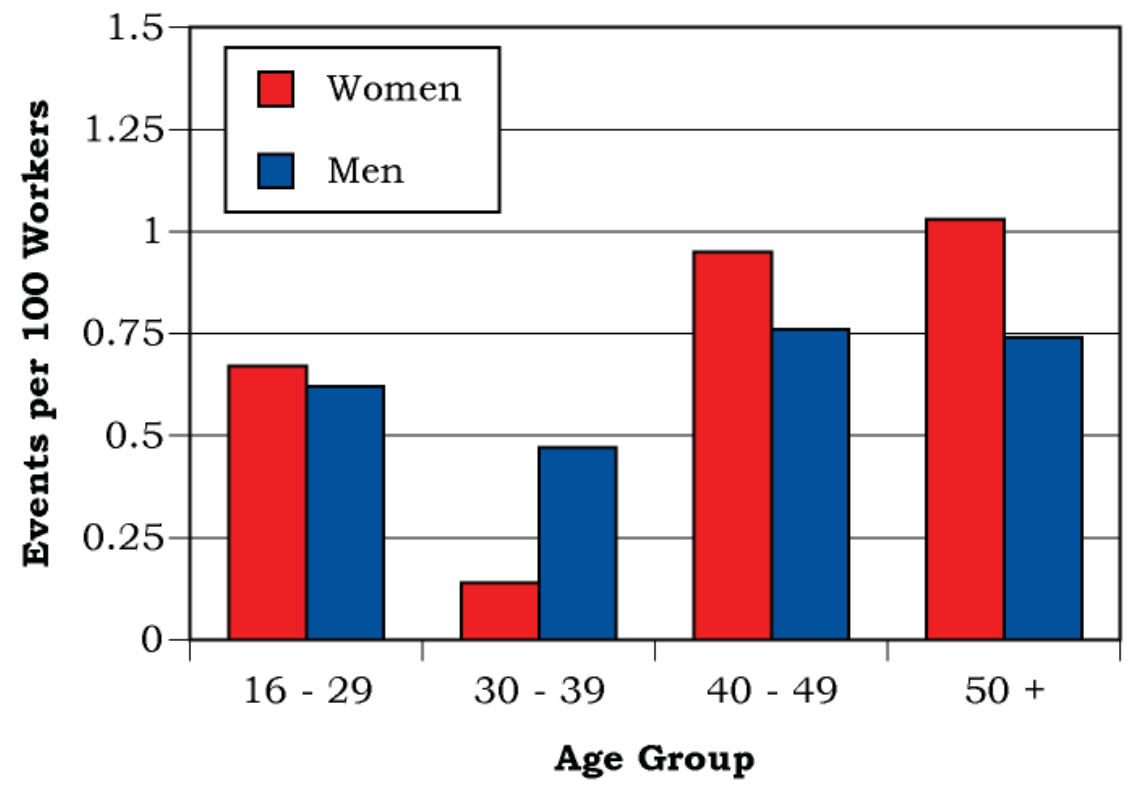


Figure 15. OSHA-Recordable Events by Job Category and Gender

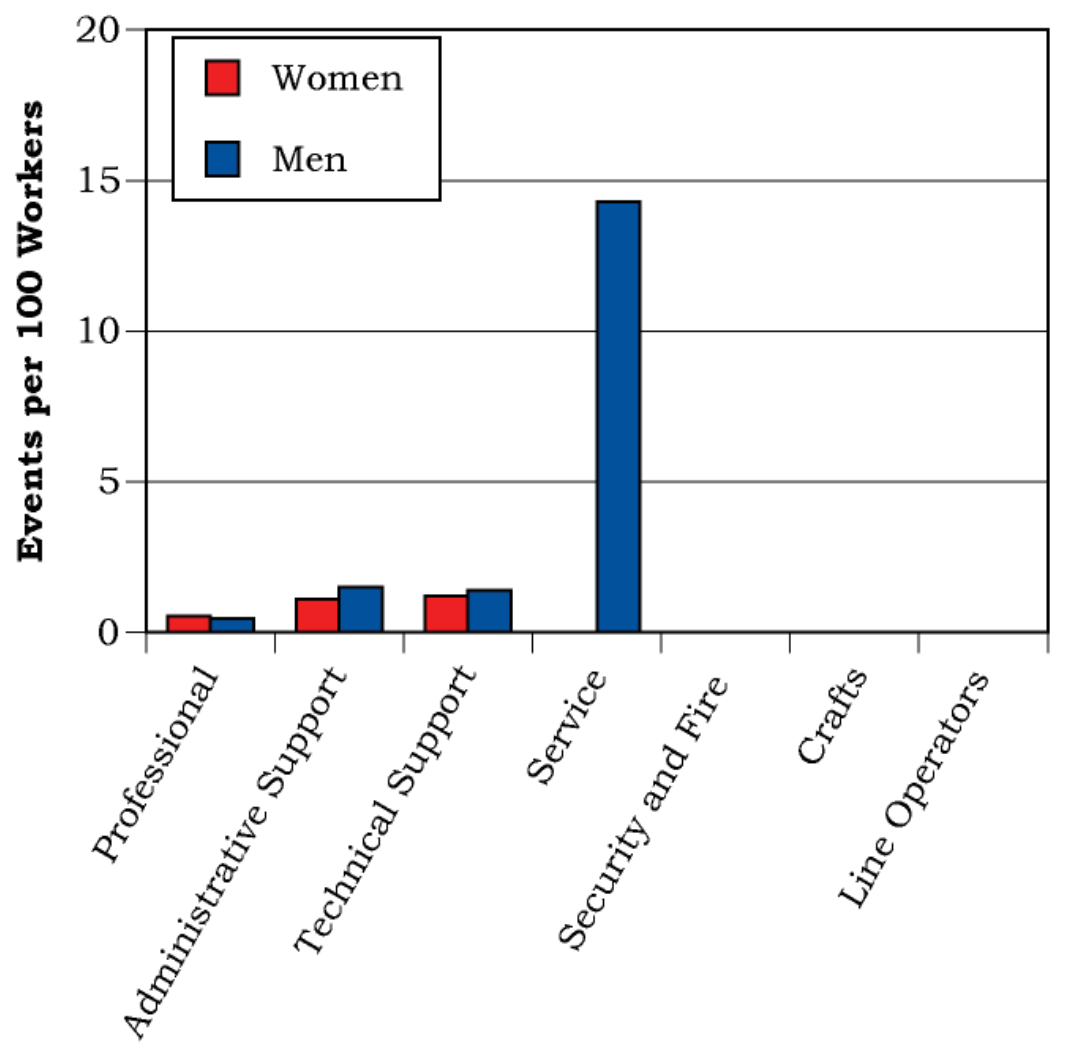

Job Category 


\section{Diagnostic and Accident Categories for OSHA-Recordable Events}

Figure 16. OSHA-Recordable Diagnoses by Diagnostic Category and Gender

\begin{tabular}{|l|c|c|}
\hline \multirow{2}{*}{ Diagnostic Category } & \multicolumn{2}{|c|}{ Gender } \\
\cline { 2 - 3 } & Women & Men \\
\hline Musculoskeletal & $\mathbf{1 5}$ & $\mathbf{1 1}$ \\
\hline Nervous System & $\mathbf{2}$ & $\mathbf{0}$ \\
\hline Psychological & $\mathbf{0}$ & $\mathbf{1}$ \\
\hline Unspecified Symptoms & $\mathbf{2 0}$ & $\mathbf{3}$ \\
\hline Injury & $\mathbf{1}$ & $\mathbf{0}$ \\
\hline Fractures - Upper Limb & $\mathbf{5}$ & $\mathbf{4}$ \\
\hline Back Sprains \& Strains & $\mathbf{1 0}$ & $\mathbf{6}$ \\
\hline Other Sprains \& Strains & $\mathbf{1}$ & $\mathbf{3}$ \\
\hline Open Wounds - Head, Neck, Trunk & $\mathbf{2}$ & $\mathbf{7}$ \\
\hline Open Wounds - Upper Limb & $\mathbf{0}$ & $\mathbf{1}$ \\
\hline Open Wounds - Lower Limb & $\mathbf{0}$ & $\mathbf{3}$ \\
\hline Superficial Injuries & $\mathbf{0}$ & $\mathbf{2}$ \\
\hline Bruises & $\mathbf{0}$ & $\mathbf{2}$ \\
\hline Foreign Bodies Entering Orifice & $\mathbf{1}$ & $\mathbf{2}$ \\
\hline Burns & $\mathbf{0}$ & $\mathbf{2}$ \\
\hline Unspecified Injuries & $\mathbf{0}$ & $\mathbf{3}$ \\
\hline Adverse Reactions to Non-Medical & & \\
\hline Substances & & \\
\hline
\end{tabular}

Figure 17. OSHA-Recordable Accidents by Type and Gender

\begin{tabular}{|l|c|c|}
\hline \multirow{2}{*}{\multicolumn{1}{|c|}{ Accident Category }} & \multicolumn{2}{|c|}{ Gender } \\
\cline { 2 - 3 } & Women & Men \\
\cline { 2 - 3 } & $\begin{array}{c}\text { Number of } \\
\text { Accidents }\end{array}$ & $\begin{array}{c}\text { Number of } \\
\text { Accidents }\end{array}$ \\
\hline Motor Vehicle Traffic & $\mathbf{0}$ & $\mathbf{1}$ \\
\hline Poisoning - Non-Medicinal & $\mathbf{0}$ & $\mathbf{2}$ \\
\hline Natural/Environmental Factors & $\mathbf{0}$ & $\mathbf{1}$ \\
\hline Submersion/Suffocation/Foreign Bodies & $\mathbf{0}$ & $\mathbf{1}$ \\
\hline Other Accidents & $\mathbf{1}$ & $\mathbf{2}$ \\
\hline Hot, Corrosive, or Caustic Material/ & $\mathbf{1}$ & $\mathbf{1}$ \\
\hline Steam & & $\mathbf{1}$ \\
\hline Repetitive Trauma & $\mathbf{0}$ & $\mathbf{7}$ \\
\hline Total & $\mathbf{1}$ & \\
\hline
\end{tabular}




\section{Rates of OSHA-Recordable Events}

Figure 18. OSHA-Recordable Rates by Age and Job Categories Among Women, All Diagnoses Combined

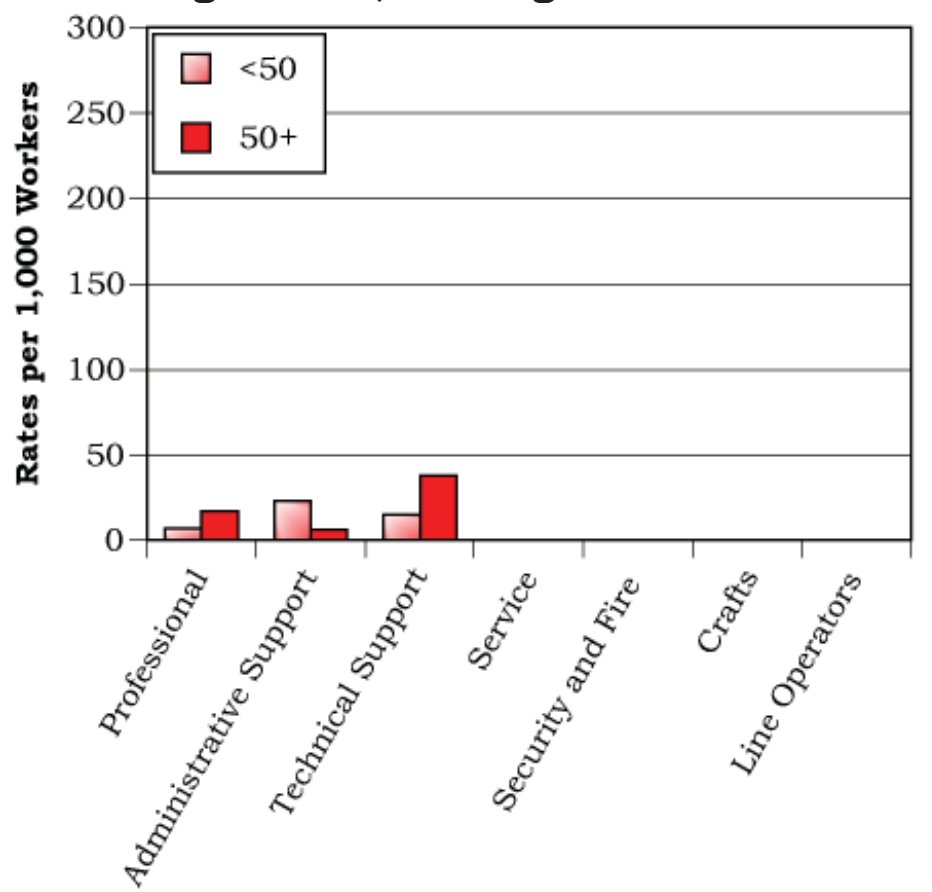

Job Category

Figure 19. OSHA-Recordable Rates by Age and Job Categories Among Men, All Diagnoses Combined

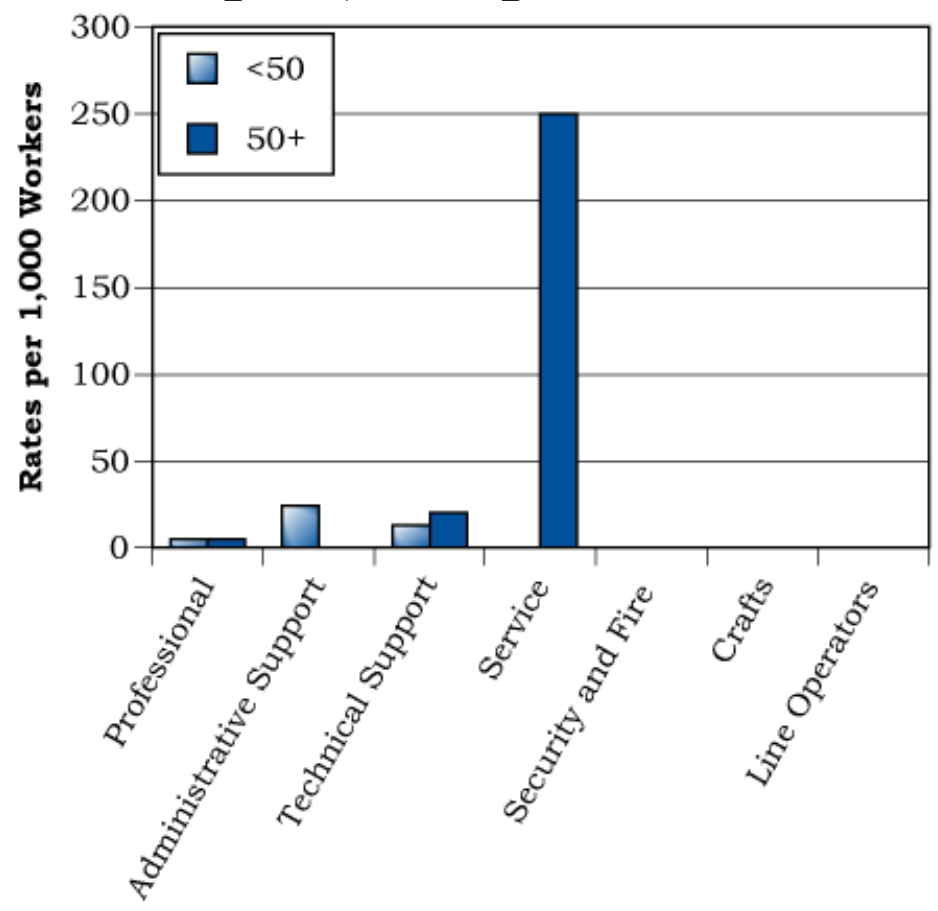

Job Category 


\section{Appendices}


Los Alamos National Laboratory 2003

Absence Data

Appendix A. Work Force by Gender, Age, and Job Category

\begin{tabular}{|c|c|c|c|c|c|c|c|c|c|c|c|}
\hline \multirow{3}{*}{ Job Category } & \multicolumn{5}{|c|}{ Women } & \multicolumn{5}{|c|}{ Men } & \multirow[b]{3}{*}{ TOTAL } \\
\hline & \multicolumn{4}{|c|}{ Age Group } & \multirow[b]{2}{*}{ TOTAL } & \multicolumn{4}{|c|}{ Age Group } & \multirow[b]{2}{*}{ TOTAL } & \\
\hline & $16-29$ & $30-39$ & $40-49$ & $50+$ & & $16-29$ & 30 - 39 & $40-49$ & $50+$ & & \\
\hline Professional & 228 & 501 & 793 & 515 & 2,037 & 405 & 1,064 & 1,688 & 1,729 & 4,886 & 6,923 \\
\hline Administrative Support & 108 & 124 & 210 & 178 & 620 & 9 & 11 & 21 & 24 & 65 & 685 \\
\hline Technical Support & 109 & 72 & 151 & 80 & 412 & 233 & 212 & 522 & 405 & 1,372 & 1,784 \\
\hline Service & 0 & 1 & 1 & 2 & 4 & 0 & 0 & 3 & 4 & 7 & 11 \\
\hline TOTAL & 445 & 698 & 1,155 & 775 & 3,073 & 647 & 1,287 & 2,234 & 2,162 & 6,330 & 9,403 \\
\hline
\end{tabular}

Appendix B. Age Distribution of the Work Force by Gender

\begin{tabular}{|c|r|r|r|r|r|r|r|r|}
\hline \multirow{4}{*}{ Year } & \multicolumn{4}{|c|}{ Women } & \multicolumn{4}{c|}{ Men } \\
\cline { 2 - 9 } & \multicolumn{3}{|c|}{$\begin{array}{c}\text { Percent Distribution by Age } \\
\text { Group }\end{array}$} & \multicolumn{4}{c|}{$\begin{array}{c}\text { Percent Distribution by Age } \\
\text { Group }\end{array}$} \\
\cline { 2 - 9 } & $\mathbf{1 6}-\mathbf{2 9}$ & $\mathbf{3 0}-\mathbf{3 9}$ & $\mathbf{4 0}-\mathbf{4 9}$ & $\mathbf{5 0}+$ & $\mathbf{1 6}-\mathbf{2 9}$ & $\mathbf{3 0}-\mathbf{3 9}$ & $\mathbf{4 0}-\mathbf{4 9}$ & $\mathbf{5 0}+$ \\
\hline $\mathbf{2 0 0 3}$ & 14.48 & 22.71 & 37.59 & 25.22 & 10.22 & 20.33 & 35.29 & 34.15 \\
\hline
\end{tabular}


Los Alamos National Laboratory 2003

Absence Data

Appendix C. Total Number of Workers Who Reported at Least One Absence by Gender, Age, and Job Category*

\begin{tabular}{|c|c|c|c|c|c|c|c|c|c|c|c|}
\hline \multirow{3}{*}{ Job Category } & \multicolumn{5}{|c|}{ Women } & \multicolumn{5}{|c|}{ Men } & \multirow[b]{3}{*}{ TOTAL } \\
\hline & \multicolumn{4}{|c|}{ Age Group } & \multirow[b]{2}{*}{ TOTAL } & \multicolumn{4}{|c|}{ Age Group } & \multirow[b]{2}{*}{ TOTAL } & \\
\hline & $16-29$ & 30 - 39 & $40-49$ & $50+$ & & $16-29$ & 30 - 39 & $40-49$ & $50+$ & & \\
\hline Professional & 2 & 16 & 59 & 41 & 118 & 8 & 17 & 68 & 77 & 170 & 288 \\
\hline Administrative Support & 3 & 8 & 23 & 16 & 50 & 0 & 1 & 1 & 3 & 5 & 55 \\
\hline Technical Support & 3 & 6 & 12 & 11 & 32 & 7 & 12 & 51 & 59 & 129 & 161 \\
\hline Service & 0 & 0 & 0 & 0 & 0 & 0 & 0 & 0 & 2 & 2 & 2 \\
\hline TOTAL & 8 & 30 & 94 & 68 & 200 & 15 & 30 & 120 & 141 & 306 & 506 \\
\hline
\end{tabular}

*Only those job categories and gender/age combinations with at least one absence appear in this table.

Appendix D. Total Number of Absences by Gender, Age, and Job Category*

\begin{tabular}{|c|c|c|c|c|c|c|c|c|c|c|c|}
\hline \multirow{3}{*}{ Job Category } & \multicolumn{5}{|c|}{ Women } & \multicolumn{5}{|c|}{ Men } & \multirow[b]{3}{*}{ TOTAL } \\
\hline & \multicolumn{4}{|c|}{ Age Group } & \multirow[b]{2}{*}{ TOTAL } & \multicolumn{4}{|c|}{ Age Group } & \multirow[b]{2}{*}{ TOTAL } & \\
\hline & $16-29$ & 30 - 39 & $40-49$ & $50+$ & & $16-29$ & $30-39$ & $40-49$ & $50+$ & & \\
\hline Professional & 2 & 18 & 65 & 51 & 136 & 9 & 19 & 70 & 79 & 177 & 313 \\
\hline Administrative Support & 3 & 9 & 27 & 17 & 56 & 0 & 1 & 2 & 3 & 6 & 62 \\
\hline Technical Support & 5 & 7 & 12 & 13 & 37 & 7 & 14 & 57 & 72 & 150 & 187 \\
\hline Service & 0 & 0 & 0 & 0 & 0 & 0 & 0 & 0 & 2 & 2 & 2 \\
\hline TOTAL & 10 & 34 & 104 & 81 & 229 & 16 & 34 & 129 & 156 & 335 & 564 \\
\hline
\end{tabular}

*Only those job categories and gender/age combinations with at least one absence appear in this table. 
Los Alamos National Laboratory 2003

Absence Data

Appendix E. Distribution of the Number of Calendar Days Missed per Absence by Gender and Age*

\begin{tabular}{|c|c|c|c|c|c|c|c|c|c|c|c|}
\hline \multirow{3}{*}{$\begin{array}{c}\# \\
\text { of } \\
\text { Calendar } \\
\text { Days }\end{array}$} & \multicolumn{5}{|c|}{ Women } & \multicolumn{5}{|c|}{ Men } & \multirow[b]{3}{*}{ TOTAL } \\
\hline & \multicolumn{4}{|c|}{ Age Group } & \multirow[b]{2}{*}{ TOTAL } & \multicolumn{4}{|c|}{ Age Group } & \multirow[b]{2}{*}{ TOTAL } & \\
\hline & $16-29$ & 30 - 39 & 40 - 49 & $50+$ & & $16-29$ & 30 - 39 & $40-49$ & $50+$ & & \\
\hline$<15$ & 10 & 25 & 65 & 48 & 148 & 14 & 24 & 90 & 97 & 225 & 373 \\
\hline $15-28$ & 0 & 6 & 15 & 16 & 37 & 2 & 7 & 25 & 30 & 64 & 101 \\
\hline $29-42$ & 0 & 0 & 8 & 3 & 11 & 0 & 1 & 4 & 9 & 14 & 25 \\
\hline $43-56$ & 0 & 0 & 7 & 4 & 11 & 0 & 0 & 3 & 7 & 10 & 21 \\
\hline $57-91$ & 0 & 3 & 7 & 5 & 15 & 0 & 0 & 1 & 6 & 7 & 22 \\
\hline $92-182$ & 0 & 0 & 1 & 4 & 5 & 0 & 1 & 2 & 4 & 7 & 12 \\
\hline $183+$ & 0 & 0 & 1 & 1 & 2 & 0 & 1 & 4 & 3 & 8 & 10 \\
\hline TOTAL & 10 & 34 & 104 & 81 & 229 & 16 & 34 & 129 & 156 & 335 & 564 \\
\hline
\end{tabular}

*Only those gender/age combinations with at least one absence appear in this table. 
Los Alamos National Laboratory 2003

Absence Data

Appendix F. Distribution of the Number of Calendar Days Missed per Absence by Gender and Job Category*

Women

\begin{tabular}{|c|c|c|c|c|c|}
\hline \multirow{2}{*}{$\begin{array}{c}\# \\
\text { of } \\
\text { Calendar } \\
\text { Days }\end{array}$} & \multicolumn{4}{|c|}{ Job Category } & \multirow[b]{2}{*}{ TOTAL } \\
\hline & Professional & $\begin{array}{c}\text { Administrative } \\
\text { Support }\end{array}$ & $\begin{array}{l}\text { Technical } \\
\text { Support }\end{array}$ & Service & \\
\hline$<15$ & 87 & 37 & 24 & 0 & 148 \\
\hline $15-28$ & 27 & 6 & 4 & 0 & 37 \\
\hline $29-42$ & 7 & 3 & 1 & 0 & 11 \\
\hline $43-56$ & 6 & 3 & 2 & 0 & 11 \\
\hline $57-91$ & 6 & 4 & 5 & 0 & 15 \\
\hline $92-182$ & 2 & 2 & 1 & 0 & 5 \\
\hline $183+$ & 1 & 1 & 0 & 0 & 2 \\
\hline TOTAL & 136 & 56 & 37 & 0 & 229 \\
\hline
\end{tabular}

Men

\begin{tabular}{|c|c|c|c|c|c|}
\hline \multirow{2}{*}{$\begin{array}{c}\# \\
\text { of } \\
\text { Calendar } \\
\text { Days }\end{array}$} & \multicolumn{4}{|c|}{ Job Category } & \multirow[b]{2}{*}{ TOTAL } \\
\hline & Professional & $\begin{array}{c}\text { Administrative } \\
\text { Support }\end{array}$ & $\begin{array}{l}\text { Technical } \\
\text { Support }\end{array}$ & Service & \\
\hline$<15$ & 120 & 3 & 100 & 2 & 225 \\
\hline $15-28$ & 37 & 3 & 24 & 0 & 64 \\
\hline $29-42$ & 6 & 0 & 8 & 0 & 14 \\
\hline $43-56$ & 6 & 0 & 4 & 0 & 10 \\
\hline $57-91$ & 1 & 0 & 6 & 0 & 7 \\
\hline $92-182$ & 5 & 0 & 2 & 0 & 7 \\
\hline $183+$ & 2 & 0 & 6 & 0 & 8 \\
\hline TOTAL & 177 & 6 & 150 & 2 & 335 \\
\hline
\end{tabular}

*Only those gender/job category combinations with at least one absence appear in this table. 
Los Alamos National Laboratory 2003

Absence Data

Appendix G. Number of Diagnoses in Each Diagnostic Category by Gender and Age*

\begin{tabular}{|c|c|c|c|c|c|c|}
\hline & & \multicolumn{5}{|c|}{ Women } \\
\hline & & \multicolumn{4}{|c|}{ Age Group } & \multirow[b]{2}{*}{ TOTAL } \\
\hline & & $16-29$ & 30 - 39 & 40 - 49 & $\mathbf{5 0}+$ & \\
\hline Diagnostic Category & ICD-9-CM Code & \multirow[b]{2}{*}{0} & \multirow[b]{2}{*}{3} & \multirow[b]{2}{*}{3} & \multirow[b]{2}{*}{5} & \multirow[b]{2}{*}{11} \\
\hline INFECTIOUS \& PARASITIC DISEASES (DIS) & 001-139 & & & & & \\
\hline -Intestinal Infectious Dis & 001-009 & 0 & 1 & 2 & 0 & 3 \\
\hline -Other Bacterial Dis & $030-041$ & 0 & 0 & 0 & 0 & 0 \\
\hline -Viral Dis with Exanthem & 050-057 & 0 & 0 & 0 & 1 & 1 \\
\hline -Arthropod-Borne Viral Dis & $060-066$ & 0 & 1 & 0 & 0 & 1 \\
\hline -Other Viral Dis \& Chlamydiae & 070-079 & 0 & 1 & 1 & 4 & 6 \\
\hline MALIGNANT NEOPLASMS & 140-208, 230-234 & 0 & 0 & 0 & 9 & 9 \\
\hline -Digestive \& Peritoneal & $150-159$ & 0 & 0 & 0 & 1 & 1 \\
\hline -Respiratory \& Intrathoracic & $160-165$ & 0 & 0 & 0 & 0 & 0 \\
\hline -Breast & 174-175 & 0 & 0 & 0 & 7 & 7 \\
\hline -Genitourinary & 179-189 & 0 & 0 & 0 & 1 & 1 \\
\hline -Other \& Unspecified Sites & 190, 193-199 & 0 & 0 & 0 & 0 & 0 \\
\hline BENIGN \& UNCERTAIN NEOPLASMS & $210-229,235-239$ & 0 & 0 & 1 & 2 & 3 \\
\hline ENDOCRINE/METABOLIC/IMMUNITY & $240-279$ & 1 & 2 & 2 & 1 & 6 \\
\hline -Thyroid Gland Disorders & $240-246$ & 1 & 1 & 1 & 1 & 4 \\
\hline -Other Endocrine Gland Dis & $250-259$ & 0 & 1 & 0 & 0 & 1 \\
\hline -Other Metabolic \& Immunity Disorders & $270-279$ & 0 & 0 & 1 & 0 & 1 \\
\hline BLOOD \& BLOOD-FORMING ORGANS & $280-289$ & 0 & 1 & 0 & 0 & 1 \\
\hline MENTAL DISORDERS & $290-319$ & 1 & 2 & 8 & 1 & 12 \\
\hline -Psychoses & $290-299$ & 0 & 0 & 1 & 0 & 1 \\
\hline -Non-Psychotic Disorders & $300-302,306-316$ & 1 & 2 & 7 & 1 & 11 \\
\hline -Alcohol Dependence & 303 & 0 & 0 & 0 & 0 & 0 \\
\hline NERVOUS SYSTEM (NS) \& SENSE ORGANS & 320-389 & 0 & 1 & 8 & 5 & 14 \\
\hline -Other Disorders of Central NS & $340-349$ & 0 & 1 & 1 & 0 & 2 \\
\hline -Disorders of Peripheral NS & $350-359$ & 0 & 0 & 7 & 2 & 9 \\
\hline -Disorders of Eye & $360-379$ & 0 & 0 & 0 & 1 & 1 \\
\hline -Dis of Ear \& Mastoid & $380-389$ & 0 & 0 & 0 & 2 & 2 \\
\hline CIRCULATORY SYSTEM & $390-459$ & 0 & 0 & 2 & 4 & 6 \\
\hline -Chronic Rheumatic Heart Dis & 393-398 & 0 & 0 & 0 & 0 & 0 \\
\hline -Hypertensive Dis & $401-405$ & 0 & 0 & 0 & 3 & 3 \\
\hline -Ischemic Heart Dis & $410-414$ & 0 & 0 & 1 & 0 & 1 \\
\hline -Dis of Pulmonary Circulation & $415-417$ & 0 & 0 & 0 & 1 & 1 \\
\hline -Other Heart Dis & $420-429$ & 0 & 0 & 0 & 0 & 0 \\
\hline -Dis of Arteries \& Capillaries & $440-448$ & 0 & 0 & 1 & 0 & 1 \\
\hline -Dis of Veins, Lymphatics, Other & $451-459$ & 0 & 0 & 0 & 0 & 0 \\
\hline RESPIRATORY SYSTEM & $460-519$ & 0 & 11 & 26 & 10 & 47 \\
\hline -Acute Respiratory Infections & $460-466$ & 0 & 2 & 6 & 3 & 11 \\
\hline
\end{tabular}

(Continued)

*Only those diagnostic categories and gender/age combinations with at least one occurrence appear in this table. 
Los Alamos National Laboratory 2003

Absence Data

Appendix G. Number of Diagnoses in Each Diagnostic Category by Gender and Age*

\begin{tabular}{|c|c|c|c|c|c|c|c|}
\hline & & \multicolumn{5}{|c|}{ Men } & \multirow[b]{3}{*}{ TOTAL } \\
\hline & & \multicolumn{4}{|c|}{ Age Group } & \multirow[b]{2}{*}{ TOTAL } & \\
\hline & & $16-29$ & $30-39$ & $40-49$ & $50+$ & & \\
\hline Diagnostic Category & ICD-9-CM Code & & & & & & \\
\hline INFECTIOUS \& PARASITIC DISEASES (DIS) & 001-139 & 0 & 3 & 7 & 6 & 16 & 27 \\
\hline -Intestinal Infectious Dis & 001-009 & 0 & 0 & 1 & 0 & 1 & 4 \\
\hline -Other Bacterial Dis & $030-041$ & 0 & 1 & 0 & 0 & 1 & 1 \\
\hline -Viral Dis with Exanthem & 050-057 & 0 & 0 & 3 & 2 & 5 & 6 \\
\hline -Arthropod-Borne Viral Dis & $060-066$ & 0 & 0 & 0 & 0 & 0 & 1 \\
\hline -Other Viral Dis \& Chlamydiae & 070-079 & 0 & 2 & 3 & 4 & 9 & 15 \\
\hline MALIGNANT NEOPLASMS & $140-208,230-234$ & 0 & 0 & 4 & 3 & 7 & 16 \\
\hline -Digestive \& Peritoneal & 150-159 & 0 & 0 & 1 & 0 & 1 & 2 \\
\hline -Respiratory \& Intrathoracic & $160-165$ & 0 & 0 & 0 & 1 & 1 & 1 \\
\hline -Breast & $174-175$ & 0 & 0 & 0 & 0 & 0 & 7 \\
\hline -Genitourinary & 179-189 & 0 & 0 & 1 & 2 & 3 & 4 \\
\hline -Other \& Unspecified Sites & 190, 193-199 & 0 & 0 & 2 & 0 & 2 & 2 \\
\hline BENIGN \& UNCERTAIN NEOPLASMS & 210-229, 235-239 & 0 & 0 & 1 & 2 & 3 & 6 \\
\hline ENDOCRINE/METABOLIC/IMMUNITY & 240-279 & 0 & 1 & 3 & 2 & 6 & 12 \\
\hline -Thyroid Gland Disorders & $240-246$ & 0 & 0 & 2 & 0 & 2 & 6 \\
\hline -Other Endocrine Gland Dis & $250-259$ & 0 & 1 & 0 & 1 & 2 & 3 \\
\hline -Other Metabolic \& Immunity Disorders & 270-279 & 0 & 0 & 1 & 1 & 2 & 3 \\
\hline BLOOD \& BLOOD-FORMING ORGANS & 280-289 & 0 & 0 & 0 & 0 & 0 & 1 \\
\hline \begin{tabular}{|l} 
MENTAL DISORDERS \\
\end{tabular} & 290-319 & 0 & 3 & 5 & 3 & 11 & 23 \\
\hline -Psychoses & 290-299 & 0 & 1 & 0 & 1 & 2 & 3 \\
\hline -Non-Psychotic Disorders & $300-302,306-316$ & 0 & 1 & 5 & 2 & 8 & 19 \\
\hline -Alcohol Dependence & 303 & 0 & 1 & 0 & 0 & 1 & 1 \\
\hline NERVOUS SYSTEM (NS) \& SENSE ORGANS & 320-389 & 1 & 3 & 4 & 4 & 12 & 26 \\
\hline -Other Disorders of Central NS & $340-349$ & 0 & 0 & 1 & 0 & 1 & 3 \\
\hline -Disorders of Peripheral NS & $350-359$ & 1 & 1 & 0 & 2 & 4 & 13 \\
\hline -Disorders of Eye & $360-379$ & 0 & 1 & 0 & 2 & 3 & 4 \\
\hline -Dis of Ear \& Mastoid & $380-389$ & 0 & 1 & 3 & 0 & 4 & 6 \\
\hline CIRCULATORY SYSTEM & $390-459$ & 0 & 1 & 9 & 22 & 32 & 38 \\
\hline -Chronic Rheumatic Heart Dis & 393-398 & 0 & 0 & 1 & 0 & 1 & 1 \\
\hline -Hypertensive Dis & $401-405$ & 0 & 1 & 1 & 1 & 3 & 6 \\
\hline -Ischemic Heart Dis & $410-414$ & 0 & 0 & 6 & 14 & 20 & 21 \\
\hline -Dis of Pulmonary Circulation & 415-417 & 0 & 0 & 0 & 0 & 0 & 1 \\
\hline -Other Heart Dis & $420-429$ & 0 & 0 & 1 & 5 & 6 & 6 \\
\hline -Dis of Arteries \& Capillaries & $440-448$ & 0 & 0 & 0 & 1 & 1 & 2 \\
\hline -Dis of Veins, Lymphatics, Other & 451-459 & 0 & 0 & 0 & 1 & 1 & 1 \\
\hline RESPIRATORY SYSTEM & 460-519 & 10 & 12 & 33 & 50 & 105 & 152 \\
\hline -Acute Respiratory Infections & $460-466$ & 3 & 4 & 10 & 17 & 34 & 45 \\
\hline
\end{tabular}

(Continued)

*Only those diagnostic categories and gender/age combinations with at least one occurrence appear in this table. 
Los Alamos National Laboratory 2003

Absence Data

Appendix G. Number of Diagnoses in Each Diagnostic Category by Gender and Age*

\begin{tabular}{|c|c|c|c|c|c|c|}
\hline & & \multicolumn{5}{|c|}{ Women } \\
\hline & & \multicolumn{4}{|c|}{ Age Group } & \multirow[b]{2}{*}{ TOTAL } \\
\hline & & $16-29$ & $30-39$ & $40-49$ & $50+$ & \\
\hline Diagnostic Category & $\begin{array}{l}\text { ICD-9-CM } \\
\text { Code }\end{array}$ & & & & & \\
\hline -Other Dis Upper Respiratory Tract & $470-478$ & 0 & 2 & 6 & 2 & 10 \\
\hline -Pneumonia \& Influenza & $480-487$ & 0 & 6 & 9 & 4 & 19 \\
\hline -Chronic Obstructive Dis & $490-496$ & 0 & 1 & 5 & 1 & 7 \\
\hline -Other Respiratory Dis & $510-519$ & 0 & 0 & 0 & 0 & 0 \\
\hline DIGESTIVE SYSTEM & $520-579$ & 0 & 2 & 11 & 17 & 30 \\
\hline -Oral Cavity, Saliva Glands, Jaw & $520-529$ & 0 & 0 & 1 & 0 & 1 \\
\hline -Esophagus, Stomach, Duodenum & $530-537$ & 0 & 0 & 0 & 1 & 1 \\
\hline -Appendicitis & $540-543$ & 0 & 0 & 0 & 2 & 2 \\
\hline -Hernia & $550-553$ & 0 & 0 & 0 & 1 & 1 \\
\hline -Enteritis, Colitis & $555-558$ & 0 & 0 & 1 & 0 & 1 \\
\hline -Other Intestinal Dis & \begin{tabular}{|l|}
$560-569$ \\
\end{tabular} & 0 & 0 & 0 & 1 & 1 \\
\hline -Other Digestive Dis & $570-579$ & 0 & 2 & 9 & 12 & 23 \\
\hline GENITOURINARY SYSTEM & $580-629$ & 3 & 7 & 18 & 6 & 34 \\
\hline -Nephritis, Nephrosis & $580-589$ & 0 & 1 & 0 & 0 & 1 \\
\hline -Other Urinary Dis & $590-599$ & 0 & 3 & 0 & 0 & 3 \\
\hline -Male Genital Organ Dis & $600-608$ & 0 & 0 & 0 & 0 & 0 \\
\hline -Breast Disorders & $610-611$ & 0 & 0 & 0 & 1 & 1 \\
\hline -Other Female Disorders & $617-629$ & 3 & 3 & 18 & 5 & 29 \\
\hline PREGNANCY \& CHILDBIRTH & $630-677$ & 0 & 0 & 1 & 0 & 1 \\
\hline -Ectopic \& Molar Pregnancy & $630-633$ & 0 & 0 & 1 & 0 & 1 \\
\hline SKIN \& SUBCUTANEOUS TISSUE & $680-709$ & 0 & 0 & 0 & 0 & 0 \\
\hline -Infections & $680-686$ & 0 & 0 & 0 & 0 & 0 \\
\hline -Other & 700-709 & 0 & 0 & 0 & 0 & 0 \\
\hline MUSCULOSKELETAL \& CONNECTIVE TISSUE & 710-739 & 4 & 8 & 26 & 22 & 60 \\
\hline -Arthropathies & $710-719$ & 1 & 7 & 12 & 12 & 32 \\
\hline -Dorsopathies & 720-724 & 2 & 1 & 11 & 4 & 18 \\
\hline -Rheumatism, Excluding Back & 725-729 & 1 & 0 & 3 & 6 & 10 \\
\hline -Other Dis \& Acquired Deformities & 730-739 & 0 & 0 & 0 & 0 & 0 \\
\hline SYMPTOMS, SIGNS, \& ILL-DEFINED CONDITIONS & $780-799$ & 1 & 3 & 9 & 2 & 15 \\
\hline -Symptoms & 780-789 & 1 & 3 & 8 & 2 & 14 \\
\hline -Ill-Defined \& Unknown Causes & 797-799 & 0 & 0 & 1 & 0 & 1 \\
\hline INJURY \& POISONING & $800-999$ & 1 & 2 & 10 & 8 & 21 \\
\hline -Fracture - Neck, Trunk & 805-809 & 0 & 0 & 0 & 0 & 0 \\
\hline -Fracture - Upper Limb & 810-819 & 0 & 0 & 1 & 2 & 3 \\
\hline -Fracture - Lower Limb & $820-829$ & 0 & 0 & 2 & 2 & 4 \\
\hline -Sprains \& Strains - Back & 846-847 & 0 & 0 & 0 & 0 & 0 \\
\hline
\end{tabular}

(Continued)

*Only those diagnostic categories and gender/age combinations with at least one occurrence appear in this table. 
Los Alamos National Laboratory 2003

Absence Data

Appendix G. Number of Diagnoses in Each Diagnostic Category by Gender and Age*

\begin{tabular}{|c|c|c|c|c|c|c|c|}
\hline & & \multicolumn{5}{|c|}{ Men } & \multirow[b]{3}{*}{ TOTAL } \\
\hline & & \multicolumn{4}{|c|}{ Age Group } & \multirow[b]{2}{*}{ TOTAL } & \\
\hline & & $16-29$ & $30-39$ & $40-49$ & $50+$ & & \\
\hline Diagnostic Category & $\begin{array}{l}\text { ICD-9-CM } \\
\text { Code }\end{array}$ & & & & & & \\
\hline -Other Dis Upper Respiratory Tract & $470-478$ & 2 & 1 & 10 & 8 & 21 & 31 \\
\hline -Pneumonia \& Influenza & $480-487$ & 2 & 7 & 10 & 15 & 34 & 53 \\
\hline -Chronic Obstructive Dis & $490-496$ & 3 & 0 & 3 & 7 & 13 & 20 \\
\hline -Other Respiratory Dis & $510-519$ & 0 & 0 & 0 & 3 & 3 & 3 \\
\hline DIGESTIVE SYSTEM & $520-579$ & 0 & 5 & 23 & 29 & 57 & 87 \\
\hline -Oral Cavity, Saliva Glands, Jaw & $520-529$ & 0 & 1 & 1 & 2 & 4 & 5 \\
\hline -Esophagus, Stomach, Duodenum & $530-537$ & 0 & 1 & 0 & 0 & 1 & 2 \\
\hline -Appendicitis & $540-543$ & 0 & 0 & 1 & 2 & 3 & 5 \\
\hline -Hernia & $550-553$ & 0 & 3 & 13 & 12 & 28 & 29 \\
\hline -Enteritis, Colitis & $555-558$ & 0 & 0 & 0 & 1 & 1 & 2 \\
\hline -Other Intestinal Dis & $560-569$ & 0 & 0 & 6 & 6 & 12 & 13 \\
\hline -Other Digestive Dis & $570-579$ & 0 & 0 & 2 & 6 & 8 & 31 \\
\hline GENITOURINARY SYSTEM & $580-629$ & 0 & 0 & 1 & 7 & 8 & 42 \\
\hline -Nephritis, Nephrosis & $580-589$ & 0 & 0 & 0 & 1 & 1 & 2 \\
\hline -Other Urinary Dis & $590-599$ & 0 & 0 & 0 & 0 & 0 & 3 \\
\hline -Male Genital Organ Dis & $600-608$ & 0 & 0 & 1 & 6 & 7 & 7 \\
\hline -Breast Disorders & 610-611 & 0 & 0 & 0 & 0 & 0 & 1 \\
\hline -Other Female Disorders & 617-629 & 0 & 0 & 0 & 0 & 0 & 29 \\
\hline PREGNANCY \& CHILDBIRTH & 630-677 & 0 & 0 & 0 & 0 & 0 & 1 \\
\hline -Ectopic \& Molar Pregnancy & $630-633$ & 0 & 0 & 0 & 0 & 0 & 1 \\
\hline SKIN \& SUBCUTANEOUS TISSUE & 680-709 & 0 & 0 & 1 & 2 & 3 & 3 \\
\hline -Infections & $680-686$ & 0 & 0 & 0 & 2 & 2 & 2 \\
\hline -Other & 700-709 & 0 & 0 & 1 & 0 & 1 & 1 \\
\hline MUSCULOSKELETAL \& CONNECTIVE TISSUE & $710-739$ & 4 & 9 & 29 & 22 & 64 & 124 \\
\hline -Arthropathies & $710-719$ & 3 & 6 & 19 & 13 & 41 & 73 \\
\hline -Dorsopathies & 720-724 & 1 & 2 & 8 & 6 & 17 & 35 \\
\hline -Rheumatism, Excluding Back & 725-729 & 0 & 1 & 1 & 2 & 4 & 14 \\
\hline -Other Dis \& Acquired Deformities & 730-739 & 0 & 0 & 1 & 1 & 2 & 2 \\
\hline SYMPTOMS, SIGNS, \& ILL-DEFINED CONDITIONS & 780-799 & 0 & 2 & 9 & 12 & 23 & 38 \\
\hline -Symptoms & 780-789 & 0 & 2 & 9 & 12 & 23 & 37 \\
\hline -Ill-Defined \& Unknown Causes & 797-799 & 0 & 0 & 0 & 0 & 0 & 1 \\
\hline INJURY \& POISONING & $800-999$ & 4 & 6 & 19 & 25 & 54 & 75 \\
\hline -Fracture - Neck, Trunk & 805-809 & 0 & 0 & 0 & 2 & 2 & 2 \\
\hline -Fracture - Upper Limb & 810-819 & 0 & 0 & 3 & 4 & 7 & 10 \\
\hline -Fracture - Lower Limb & 820-829 & 0 & 1 & 3 & 1 & 5 & 9 \\
\hline -Sprains \& Strains - Back & 846-847 & 0 & 0 & 1 & 1 & 2 & 2 \\
\hline
\end{tabular}

(Continued)

*Only those diagnostic categories and gender/age combinations with at least one occurrence appear in this table. 
Los Alamos National Laboratory 2003

Absence Data

Appendix G. Number of Diagnoses in Each Diagnostic Category by Gender and Age*

\begin{tabular}{|c|c|c|c|c|c|c|}
\hline & & \multicolumn{5}{|c|}{ Women } \\
\hline & & \multicolumn{4}{|c|}{ Age Group } & \multirow[b]{2}{*}{ TOTAL } \\
\hline & & $16-29$ & $30-39$ & $40-49$ & $50+$ & \\
\hline Diagnostic Category & \begin{tabular}{|l|} 
ICD-9-CM \\
Code
\end{tabular} & & & & & \\
\hline -Sprains \& Strains - Other & $840-845,848$ & 0 & 0 & 1 & 2 & 3 \\
\hline -Intracranial Injury & $850-854$ & 0 & 0 & 1 & 0 & 1 \\
\hline -Internal Injury - Thorax, Abdomen, Pelvis & $860-869$ & 0 & 0 & 0 & 0 & 0 \\
\hline -Open Wound - Upper Limb & 880-887 & 0 & 0 & 0 & 0 & 0 \\
\hline -Superficial Injury & 910-919 & 0 & 0 & 1 & 0 & 1 \\
\hline -Contusion & 920-924 & 1 & 1 & 0 & 0 & 2 \\
\hline -Burns & 940-949 & 0 & 0 & 0 & 0 & 0 \\
\hline -Complications \& Unspecified Injuries & 958-959 & 0 & 1 & 3 & 1 & 5 \\
\hline -Toxic Effects - Non-medicinal & 980-989 & 0 & 0 & 0 & 0 & 0 \\
\hline -Unspecified Effects - External Causes & 990-995 & 0 & 0 & 1 & 1 & 2 \\
\hline -Complications of Surgical/Medical Care & 996-999 & 0 & 0 & 0 & 0 & 0 \\
\hline HEALTH STATUS/HEALTH SERVICE CONTACT & V01-V82 & 0 & 1 & 0 & 0 & 1 \\
\hline -Health Services Reproduction/Development & V20-V29 & 0 & 0 & 0 & 0 & 0 \\
\hline -Other Circumstances & V60-V69 & 0 & 1 & 0 & 0 & 1 \\
\hline
\end{tabular}

*Only those diagnostic categories and gender/age combinations with at least one occurrence appear in this table. 
Los Alamos National Laboratory 2003

Absence Data

Appendix G. Number of Diagnoses in Each Diagnostic Category by Gender and Age*

\begin{tabular}{|c|c|c|c|c|c|c|c|}
\hline & & \multicolumn{5}{|c|}{ Men } & \multirow[b]{3}{*}{ TOTAL } \\
\hline & & \multicolumn{4}{|c|}{ Age Group } & \multirow[b]{2}{*}{ TOTAL } & \\
\hline & & $16-29$ & 30 - 39 & $40-49$ & $50+$ & & \\
\hline Diagnostic Category & \begin{tabular}{|l|} 
ICD-9-CM \\
Code
\end{tabular} & & & & & & \\
\hline -Sprains \& Strains - Other & $840-845,848$ & 1 & 0 & 3 & 8 & 12 & 15 \\
\hline -Intracranial Injury & $850-854$ & 0 & 0 & 0 & 0 & 0 & 1 \\
\hline -Internal Injury - Thorax, Abdomen, Pelvis & $860-869$ & 0 & 3 & 0 & 0 & 3 & 3 \\
\hline -Open Wound - Upper Limb & 880-887 & 0 & 1 & 1 & 0 & 2 & 2 \\
\hline -Superficial Injury & $910-919$ & 0 & 0 & 1 & 1 & 2 & 3 \\
\hline -Contusion & $920-924$ & 0 & 0 & 1 & 2 & 3 & 5 \\
\hline -Burns & 940-949 & 1 & 0 & 0 & 0 & 1 & 1 \\
\hline -Complications \& Unspecified Injuries & 958-959 & 2 & 0 & 4 & 4 & 10 & 15 \\
\hline -Toxic Effects - Non-medicinal & 980-989 & 0 & 0 & 1 & 0 & 1 & 1 \\
\hline -Unspecified Effects - External Causes & $990-995$ & 0 & 1 & 0 & 1 & 2 & 4 \\
\hline -Complications of Surgical/Medical Care & 996-999 & 0 & 0 & 1 & 1 & 2 & 2 \\
\hline HEALTH STATUS/HEALTH SERVICE CONTACT & V01-V82 & 1 & 1 & 0 & 0 & 2 & 3 \\
\hline -Health Services Reproduction/Development & V20-V29 & 1 & 1 & 0 & 0 & 2 & 2 \\
\hline -Other Circumstances & V60-V69 & 0 & 0 & 0 & 0 & \begin{tabular}{l|l}
0 \\
\end{tabular} & 1 \\
\hline
\end{tabular}

\begin{tabular}{|c|c|c|c|c|c|c|c|c|c|c|c|}
\hline & \multicolumn{5}{|c|}{ Women } & \multicolumn{5}{|c|}{ Men } & \multirow[b]{3}{*}{ TOTAL } \\
\hline & \multicolumn{4}{|c|}{ Age Group } & \multirow[b]{2}{*}{ TOTAL } & \multicolumn{4}{|c|}{ Age Group } & \multirow[b]{2}{*}{ TOTAL } & \\
\hline & $16-29$ & $30-39$ & $40-49$ & $50+$ & & $16-29$ & $30-39$ & $40-49$ & $50+$ & & \\
\hline $\begin{array}{l}\text { Diagnostic } \\
\text { Category }\end{array}$ & & & & & & & & & & & \\
\hline Total & 11 & 43 & 125 & 92 & 271 & 20 & 46 & 148 & 189 & 403 & 674 \\
\hline
\end{tabular}

*Only those diagnostic categories and gender/age combinations with at least one occurrence appear in this table. 
Los Alamos National Laboratory 2003

Absence Data

Appendix H. Total Number of Calendar Days Absent in Each Diagnostic Category by Gender and Age*

\begin{tabular}{|c|c|c|c|c|c|c|}
\hline & & \multicolumn{5}{|c|}{ Women } \\
\hline & & \multicolumn{4}{|c|}{ Age Group } & \multirow[b]{2}{*}{ TOTAL } \\
\hline & & $16-29$ & 30 - 39 & $40-49$ & $50+$ & \\
\hline Diagnostic Category & ICD-9-CM Code & \multirow[b]{2}{*}{0} & \multirow[b]{2}{*}{23} & \multirow[b]{2}{*}{30} & \multirow[b]{2}{*}{56} & \multirow[b]{2}{*}{109} \\
\hline INFECTIOUS \& PARASITIC DISEASES (DIS) & 001-139 & & & & & \\
\hline MALIGNANT NEOPLASMS & 140-208, 230-234 & 0 & 0 & 0 & 284 & 284 \\
\hline BENIGN \& UNCERTAIN NEOPLASMS & 210-229, 235-239 & 0 & 0 & 32 & 39 & 71 \\
\hline ENDOCRINE/METABOLIC/IMMUNITY & $240-279$ & 9 & 38 & 31 & 26 & 104 \\
\hline BLOOD \& BLOOD-FORMING ORGANS & $280-289$ & 0 & 16 & 0 & 0 & 16 \\
\hline MENTAL DISORDERS & $290-319$ & 9 & 95 & 163 & 14 & 281 \\
\hline NERVOUS SYSTEM (NS) \& SENSE ORGANS & $320-389$ & 0 & 9 & 132 & 61 & 202 \\
\hline CIRCULATORY SYSTEM & $390-459$ & 0 & 0 & 74 & 287 & 361 \\
\hline RESPIRATORY SYSTEM & $460-519$ & 0 & 80 & 333 & 72 & 485 \\
\hline DIGESTIVE SYSTEM & $520-579$ & 0 & 104 & 158 & 417 & 679 \\
\hline GENITOURINARY SYSTEM & $580-629$ & 34 & 142 & 539 & 309 & 1,024 \\
\hline PREGNANCY \& CHILDBIRTH & $630-677$ & 0 & 0 & 20 & 0 & 20 \\
\hline SKIN \& SUBCUTANEOUS TISSUE & 680-709 & 0 & 0 & 0 & 0 & 0 \\
\hline MUSCULOSKELETAL \& CONNECTIVE TISSUE & 710-739 & 40 & 76 & 747 & 437 & 1,300 \\
\hline SYMPTOMS, SIGNS, \& ILL-DEFINED CONDITIONS & 780-799 & 4 & 25 & 176 & 23 & 228 \\
\hline INJURY \& POISONING & $800-999$ & 7 & 15 & 136 & 182 & 340 \\
\hline
\end{tabular}

\footnotetext{
*Absences with >1 ICD-9-CM code in the same diagnostic category were counted only once. Only those diagnostic categories and gender/age combinations with at least one occurrence appear in this table.
} 
Los Alamos National Laboratory 2003

Absence Data

Appendix H. Total Number of Calendar Days Absent in Each Diagnostic Category by Gender and Age*

\begin{tabular}{|c|c|c|c|c|c|c|c|}
\hline & & \multicolumn{5}{|c|}{ Men } & \multirow[b]{3}{*}{ TOTAL } \\
\hline & & \multicolumn{4}{|c|}{ Age Group } & \multirow[b]{2}{*}{ TOTAL } & \\
\hline & & $16-29$ & $30-39$ & $40-49$ & $\mathbf{5 0 +}$ & & \\
\hline Diagnostic Category & ICD-9-CM Code & \multirow[b]{2}{*}{0} & \multirow[b]{2}{*}{28} & \multirow[b]{2}{*}{85} & \multirow[b]{2}{*}{67} & \multirow[b]{2}{*}{180} & \multirow[b]{2}{*}{289} \\
\hline INFECTIOUS \& PARASITIC DISEASES (DIS) & $001-139$ & & & & & & \\
\hline MALIGNANT NEOPLASMS & $140-208,230-234$ & 0 & 0 & 1,166 & 124 & 1,290 & 1,574 \\
\hline BENIGN \& UNCERTAIN NEOPLASMS & 210-229, 235-239 & 0 & 0 & 29 & 402 & 431 & 502 \\
\hline ENDOCRINE/METABOLIC/IMMUNITY & $240-279$ & 0 & 21 & 38 & 14 & 73 & 177 \\
\hline BLOOD \& BLOOD-FORMING ORGANS & $280-289$ & 0 & 0 & 0 & 0 & 0 & 16 \\
\hline MENTAL DISORDERS & 290-319 & 0 & 213 & 829 & 242 & 1,284 & 1,565 \\
\hline NERVOUS SYSTEM (NS) \& SENSE ORGANS & $320-389$ & 14 & 59 & 31 & 65 & 169 & 371 \\
\hline CIRCULATORY SYSTEM & $390-459$ & 0 & 11 & 219 & 1,302 & 1,532 & 1,893 \\
\hline RESPIRATORY SYSTEM & $460-519$ & 66 & 102 & 621 & 445 & 1,234 & 1,719 \\
\hline DIGESTIVE SYSTEM & $520-579$ & 0 & 66 & 275 & 424 & 765 & 1,444 \\
\hline GENITOURINARY SYSTEM & $580-629$ & 0 & 0 & 8 & 214 & 222 & 1,246 \\
\hline PREGNANCY \& CHILDBIRTH & $630-677$ & 0 & 0 & 0 & 0 & 0 & 20 \\
\hline SKIN \& SUBCUTANEOUS TISSUE & $680-709$ & 0 & 0 & 18 & 60 & 78 & 78 \\
\hline MUSCULOSKELETAL \& CONNECTIVE TISSUE & 710-739 & 54 & 185 & 636 & 615 & 1,490 & 2,790 \\
\hline SYMPTOMS, SIGNS, \& ILL-DEFINED CONDITIONS & 780-799 & 0 & 14 & 107 & 233 & 354 & 582 \\
\hline INJURY \& POISONING & $800-999$ & 23 & 57 & 166 & 563 & 809 & 1,149 \\
\hline
\end{tabular}

\footnotetext{
*Absences with >1 ICD-9-CM code in the same diagnostic category were counted only once. Only those diagnostic categories and gender/age combinations with at least one occurrence appear in this table.
} 
Los Alamos National Laboratory 2003

Absence Data

Appendix I. Number of Diagnoses in Each Diagnostic Category by Gender and Job Category*

\begin{tabular}{|c|c|c|c|c|c|}
\hline & & \multicolumn{4}{|c|}{ Women } \\
\hline & & \multicolumn{3}{|c|}{ Job Category } & \multirow[b]{2}{*}{ TOTAL } \\
\hline & & Professional & $\begin{array}{c}\text { Administrative } \\
\text { Support }\end{array}$ & $\begin{array}{l}\text { Technical } \\
\text { Support }\end{array}$ & \\
\hline Diagnostic Category & ICD-9-CM Code & \multirow[b]{2}{*}{7} & \multirow[b]{2}{*}{1} & \multirow[b]{2}{*}{3} & \multirow[b]{2}{*}{11} \\
\hline INFECTIOUS \& PARASITIC DISEASES (DIS) & 001-139 & & & & \\
\hline -Intestinal Infectious Dis & 001-009 & 2 & 0 & 1 & 3 \\
\hline -Viral Dis with Exanthem & 050-057 & 1 & 0 & 0 & 1 \\
\hline -Arthropod-Borne Viral Dis & $060-066$ & 0 & 0 & 1 & 1 \\
\hline -Other Viral Dis \& Chlamydiae & 070-079 & 4 & 1 & 1 & 6 \\
\hline MALIGNANT NEOPLASMS & 140-208, 230-234 & 8 & 0 & 1 & 9 \\
\hline -Digestive \& Peritoneal & $150-159$ & 0 & 0 & 1 & 1 \\
\hline -Breast & 174-175 & 7 & 0 & 0 & 7 \\
\hline -Genitourinary & 179-189 & 1 & 0 & 0 & 1 \\
\hline BENIGN \& UNCERTAIN NEOPLASMS & $210-229,235-239$ & 2 & 1 & 0 & 3 \\
\hline ENDOCRINE/METABOLIC/IMMUNITY & 240-279 & 1 & 1 & 4 & 6 \\
\hline -Thyroid Gland Disorders & $240-246$ & 1 & 1 & 2 & 4 \\
\hline -Other Endocrine Gland Dis & $250-259$ & 0 & 0 & 1 & 1 \\
\hline -Other Metabolic \& Immunity Disorders & $270-279$ & 0 & 0 & 1 & 1 \\
\hline BLOOD \& BLOOD-FORMING ORGANS & 280-289 & 1 & 0 & 0 & 1 \\
\hline MENTAL DISORDERS & 290-319 & 9 & 1 & 2 & 12 \\
\hline -Psychoses & 290-299 & 1 & 0 & 0 & 1 \\
\hline -Non-Psychotic Disorders & $300-302,306-316$ & 8 & 1 & 2 & 11 \\
\hline NERVOUS SYSTEM (NS) \& SENSE ORGANS & 320-389 & 7 & 6 & 1 & 14 \\
\hline -Other Disorders of Central NS & $340-349$ & 1 & 1 & 0 & 2 \\
\hline -Disorders of Peripheral NS & $350-359$ & 4 & 5 & 0 & 9 \\
\hline -Disorders of Eye & $360-379$ & 0 & 0 & 1 & 1 \\
\hline -Dis of Ear \& Mastoid & 380-389 & 2 & 0 & 0 & 2 \\
\hline CIRCULATORY SYSTEM & $390-459$ & 5 & 0 & 1 & 6 \\
\hline -Hypertensive Dis & $401-405$ & 3 & 0 & 0 & 3 \\
\hline -Ischemic Heart Dis & $410-414$ & 0 & 0 & 1 & 1 \\
\hline -Dis of Pulmonary Circulation & $415-417$ & 1 & 0 & 0 & 1 \\
\hline -Dis of Arteries \& Capillaries & $440-448$ & 1 & 0 & 0 & 1 \\
\hline RESPIRATORY SYSTEM & $460-519$ & 32 & 10 & 5 & 47 \\
\hline -Acute Respiratory Infections & $460-466$ & 7 & 2 & 2 & 11 \\
\hline -Other Dis Upper Respiratory Tract & $470-478$ & 8 & 2 & 0 & 10 \\
\hline -Pneumonia \& Influenza & $480-487$ & 12 & 5 & 2 & 19 \\
\hline -Chronic Obstructive Dis & $490-496$ & 5 & 1 & 1 & 7 \\
\hline DIGESTIVE SYSTEM & $520-579$ & 19 & 7 & 4 & 30 \\
\hline -Oral Cavity, Saliva Glands, Jaw & $520-529$ & 1 & 0 & 0 & 1 \\
\hline -Esophagus, Stomach, Duodenum & $530-537$ & 0 & 1 & 0 & 1 \\
\hline
\end{tabular}

(Continued)

*Only those diagnostic categories and gender/job category combinations with at least one occurrence appear in this table. 
Los Alamos National Laboratory 2003

Absence Data

Appendix I. Number of Diagnoses in Each Diagnostic Category by Gender and Job Category*

\begin{tabular}{|c|c|c|c|c|c|}
\hline & & \multicolumn{4}{|c|}{ Women } \\
\hline & & \multicolumn{3}{|c|}{ Job Category } & \multirow[b]{2}{*}{ TOTAL } \\
\hline & & Professional & $\begin{array}{c}\text { Administrative } \\
\text { Support }\end{array}$ & $\begin{array}{l}\text { Technical } \\
\text { Support }\end{array}$ & \\
\hline Diagnostic Category & ICD-9-CM Code & \multirow[b]{2}{*}{1} & \multirow[b]{2}{*}{1} & \multirow[b]{2}{*}{0} & \multirow[b]{2}{*}{2} \\
\hline -Appendicitis & $540-543$ & & & & \\
\hline -Hernia & $550-553$ & 0 & 1 & 0 & 1 \\
\hline -Enteritis, Colitis & $555-558$ & 1 & 0 & 0 & 1 \\
\hline -Other Intestinal Dis & $560-569$ & 0 & 1 & 0 & 1 \\
\hline -Other Digestive Dis & \begin{tabular}{|l|}
$570-579$ \\
\end{tabular} & 16 & 3 & 4 & 23 \\
\hline GENITOURINARY SYSTEM & $580-629$ & 21 & 7 & 6 & 34 \\
\hline -Nephritis, Nephrosis & $580-589$ & 1 & 0 & 0 & 1 \\
\hline -Other Urinary Dis & $590-599$ & 1 & 1 & 1 & 3 \\
\hline -Breast Disorders & $610-611$ & 1 & 0 & 0 & 1 \\
\hline -Other Female Disorders & $617-629$ & 18 & 6 & 5 & 29 \\
\hline PREGNANCY \& CHILDBIRTH & $630-677$ & 1 & 0 & 0 & 1 \\
\hline -Ectopic \& Molar Pregnancy & $630-633$ & 1 & 0 & 0 & 1 \\
\hline MUSCULOSKELETAL \& CONNECTIVE TISSUE & 710-739 & 32 & 20 & 8 & 60 \\
\hline -Arthropathies & 710-719 & 17 & 10 & 5 & 32 \\
\hline -Dorsopathies & $720-724$ & 11 & 7 & 0 & 18 \\
\hline -Rheumatism, Excluding Back & 725-729 & 4 & 3 & 3 & 10 \\
\hline SYMPTOMS, SIGNS, \& ILL-DEFINED CONDITIONS & 780-799 & 9 & 2 & 4 & 15 \\
\hline -Symptoms & 780-789 & 8 & 2 & 4 & 14 \\
\hline -Ill-Defined \& Unknown Causes & 797-799 & 1 & 0 & 0 & 1 \\
\hline INJURY \& POISONING & $800-999$ & 9 & 7 & 5 & 21 \\
\hline -Fracture - Upper Limb & 810-819 & 2 & 0 & 1 & 3 \\
\hline -Fracture - Lower Limb & $820-829$ & 1 & 1 & 2 & 4 \\
\hline -Sprains \& Strains - Other & $840-845,848$ & 2 & 1 & 0 & 3 \\
\hline -Intracranial Injury & $850-854$ & 1 & 0 & 0 & 1 \\
\hline -Superficial Injury & 910-919 & 1 & 0 & 0 & 1 \\
\hline -Contusion & $920-924$ & 1 & 1 & 0 & 2 \\
\hline -Complications \& Unspecified Injuries & 958-959 & 1 & 4 & 0 & 5 \\
\hline -Unspecified Effects - External Causes & $990-995$ & 0 & 0 & 2 & 2 \\
\hline HEALTH STATUS/HEALTH SERVICE CONTACT & V01-V82 & 1 & 0 & 0 & 1 \\
\hline -Other Circumstances & V60-V69 & 1 & 0 & 0 & 1 \\
\hline
\end{tabular}

*Only those diagnostic categories and gender/job category combinations with at least one occurrence appear in this table. 
Los Alamos National Laboratory 2003

Absence Data

Appendix I. Number of Diagnoses in Each Diagnostic Category by Gender and Job Category*

\begin{tabular}{|l|r|r|r|r|}
\hline \multirow{4}{*}{} & \multicolumn{3}{|c|}{ Women } & \\
\cline { 2 - 5 } & \multicolumn{3}{|c|}{ Job Category } & \\
\cline { 2 - 5 } & Professional & $\begin{array}{c}\text { Administrative } \\
\text { Support }\end{array}$ & $\begin{array}{l}\text { Technical } \\
\text { Support }\end{array}$ & TOTAL \\
\hline $\begin{array}{l}\text { Diagnostic } \\
\text { Category }\end{array}$ & 164 & & & \\
\cline { 1 - 4 } Total & & & 4 & 271 \\
\hline
\end{tabular}

*Only those diagnostic categories and gender/job category combinations with at least one occurrence appear in this table. 
Los Alamos National Laboratory 2003

Absence Data

Appendix I. Number of Diagnoses in Each Diagnostic Category by Gender and Job Category*

\begin{tabular}{|c|c|c|c|c|c|c|}
\hline & & \multicolumn{5}{|c|}{ Men } \\
\hline & & \multicolumn{4}{|c|}{ Job Category } & \multirow[b]{2}{*}{ TOTAL } \\
\hline & & Professional & $\begin{array}{l}\text { Administrative } \\
\text { Support }\end{array}$ & \begin{tabular}{|l|} 
Technical \\
Support
\end{tabular} & Service & \\
\hline Diagnostic Category & ICD-9-CM Code & \multirow[b]{2}{*}{8} & \multirow[b]{2}{*}{0} & \multirow[b]{2}{*}{8} & \multirow[b]{2}{*}{0} & \multirow[b]{2}{*}{16} \\
\hline INFECTIOUS \& PARASITIC DISEASES (DIS) & 001-139 & & & & & \\
\hline -Intestinal Infectious Dis & 001-009 & 1 & 0 & 0 & 0 & 1 \\
\hline -Other Bacterial Dis & 030-041 & 0 & 0 & 1 & 0 & 1 \\
\hline -Viral Dis with Exanthem & 050-057 & 2 & 0 & 3 & 0 & 5 \\
\hline -Other Viral Dis \& Chlamydiae & 070-079 & 5 & 0 & 4 & 0 & 9 \\
\hline MALIGNANT NEOPLASMS & 140-208, 230-234 & 6 & 0 & 1 & 0 & 7 \\
\hline -Digestive \& Peritoneal & $150-159$ & 0 & 0 & 1 & 0 & 1 \\
\hline -Respiratory \& Intrathoracic & $160-165$ & 1 & 0 & 0 & 0 & 1 \\
\hline -Genitourinary & 179-189 & 3 & 0 & 0 & 0 & 3 \\
\hline -Other \& Unspecified Sites & 190, 193-199 & 2 & 0 & 0 & 0 & 2 \\
\hline BENIGN \& UNCERTAIN NEOPLASMS & 210-229, 235-239 & 1 & 0 & 2 & 0 & 3 \\
\hline ENDOCRINE/METABOLIC/IMMUNITY & 240-279 & 3 & 1 & 2 & 0 & 6 \\
\hline -Thyroid Gland Disorders & $240-246$ & 2 & 0 & 0 & 0 & 2 \\
\hline -Other Endocrine Gland Dis & 250-259 & 0 & 1 & 1 & 0 & 2 \\
\hline -Other Metabolic \& Immunity Disorders & $270-279$ & 1 & 0 & 1 & 0 & 2 \\
\hline MENTAL DISORDERS & 290-319 & 3 & 0 & 8 & 0 & 11 \\
\hline -Psychoses & 290-299 & 0 & 0 & 2 & 0 & 2 \\
\hline -Non-Psychotic Disorders & $300-302,306-316$ & 3 & 0 & 5 & 0 & 8 \\
\hline -Alcohol Dependence & 303 & 0 & 0 & 1 & 0 & 1 \\
\hline NERVOUS SYSTEM (NS) \& SENSE ORGANS & 320-389 & 9 & 0 & 3 & 0 & 12 \\
\hline -Other Disorders of Central NS & 340-349 & 0 & 0 & 1 & 0 & 1 \\
\hline -Disorders of Peripheral NS & 350-359 & 3 & 0 & 1 & 0 & 4 \\
\hline -Disorders of Eye & $360-379$ & 3 & 0 & 0 & 0 & 3 \\
\hline -Dis of Ear \& Mastoid & 380-389 & 3 & 0 & 1 & 0 & 4 \\
\hline CIRCULATORY SYSTEM & $390-459$ & 13 & 0 & 19 & 0 & 32 \\
\hline -Chronic Rheumatic Heart Dis & 393-398 & 1 & 0 & 0 & 0 & 1 \\
\hline -Hypertensive Dis & $401-405$ & 2 & 0 & 1 & 0 & 3 \\
\hline -Ischemic Heart Dis & $410-414$ & 6 & 0 & 14 & 0 & 20 \\
\hline -Other Heart Dis & $420-429$ & 4 & 0 & 2 & 0 & 6 \\
\hline -Dis of Arteries \& Capillaries & $440-448$ & 0 & 0 & 1 & 0 & 1 \\
\hline -Dis of Veins, Lymphatics, Other & 451-459 & 0 & 0 & 1 & 0 & 1 \\
\hline RESPIRATORY SYSTEM & 460-519 & 56 & 1 & 46 & 2 & 105 \\
\hline -Acute Respiratory Infections & $460-466$ & 13 & 0 & 20 & 1 & 34 \\
\hline -Other Dis Upper Respiratory Tract & $470-478$ & 13 & 0 & 8 & 0 & 21 \\
\hline -Pneumonia \& Influenza & $480-487$ & 22 & 0 & 11 & 1 & 34 \\
\hline -Chronic Obstructive Dis & $490-496$ & 7 & 1 & 5 & 0 & 13 \\
\hline
\end{tabular}

(Continued)

*Only those diagnostic categories and gender/job category combinations with at least one occurrence appear in this table. 
Los Alamos National Laboratory 2003

Absence Data

Appendix I. Number of Diagnoses in Each Diagnostic Category by Gender and Job Category*

\begin{tabular}{|c|c|c|c|c|c|c|}
\hline & & \multicolumn{5}{|c|}{ Men } \\
\hline & & \multicolumn{4}{|c|}{ Job Category } & \multirow[b]{2}{*}{ TOTAI } \\
\hline & & Professional & $\begin{array}{c}\text { Administrative } \\
\text { Support }\end{array}$ & \begin{tabular}{|l|} 
Technical \\
Support
\end{tabular} & Service & \\
\hline Diagnostic Category & \begin{tabular}{|l} 
ICD-9-CM \\
Code
\end{tabular} & & & & & \\
\hline -Oral Cavity, Saliva Glands, Jaw & $520-529$ & 1 & 0 & 3 & 0 & 4 \\
\hline -Esophagus, Stomach, Duodenum & $530-537$ & 1 & 0 & 0 & 0 & 1 \\
\hline -Appendicitis & $540-543$ & 1 & 1 & 1 & 0 & 3 \\
\hline -Hernia & $550-553$ & 19 & 1 & 8 & 0 & 28 \\
\hline -Other Digestive Dis & $570-579$ & 5 & 0 & 3 & 0 & 8 \\
\hline GENITOURINARY SYSTEM & $580-629$ & 5 & 0 & 3 & 0 & 8 \\
\hline -Nephritis, Nephrosis & $580-589$ & 0 & 0 & 1 & 0 & 1 \\
\hline -Male Genital Organ Dis & $600-608$ & 5 & 0 & 2 & 0 & 7 \\
\hline SKIN \& SUBCUTANEOUS TISSUE & 680-709 & 3 & 0 & 0 & 0 & 3 \\
\hline -Infections & $680-686$ & 2 & 0 & 0 & 0 & 2 \\
\hline -Other & 700-709 & 1 & 0 & 0 & 0 & 1 \\
\hline MUSCULOSKELETAL \& CONNECTIVE TISSUE & 710-739 & 32 & 1 & 31 & 0 & 64 \\
\hline INJURY \& POISONING & $800-999$ & 29 & 1 & 23 & 1 & 54 \\
\hline -Fracture - Neck, Trunk & 805-809 & 2 & 0 & 0 & 0 & 2 \\
\hline -Fracture - Upper Limb & 810-819 & 3 & 0 & 4 & 0 & 7 \\
\hline -Fracture - Lower Limb & 820-829 & 3 & 0 & 2 & 0 & 5 \\
\hline -Sprains \& Strains - Back & 846-847 & 2 & 0 & 0 & 0 & 2 \\
\hline -Sprains \& Strains - Other & $840-845,848$ & 5 & 0 & 6 & 1 & 12 \\
\hline -Internal Injury - Thorax, Abdomen, Pelvis & $860-869$ & 0 & 0 & 3 & 0 & 3 \\
\hline -Open Wound - Upper Limb & 880-887 & 0 & 0 & 2 & 0 & 2 \\
\hline -Superficial Injury & 910-919 & 2 & 0 & 0 & 0 & 2 \\
\hline -Contusion & 920-924 & 3 & 0 & 0 & 0 & 3 \\
\hline -Burns & 940-949 & 1 & 0 & 0 & 0 & 1 \\
\hline -Complications \& Unspecified Injuries & 958-959 & 6 & 0 & 4 & 0 & 10 \\
\hline -Toxic Effects - Non-medicinal & 980-989 & 0 & 0 & 1 & 0 & 1 \\
\hline
\end{tabular}

(Continued)

*Only those diagnostic categories and gender/job category combinations with at least one occurrence appear in this table. 
Los Alamos National Laboratory 2003

Absence Data

Appendix I. Number of Diagnoses in Each Diagnostic Category by Gender and Job Category*

\begin{tabular}{|c|c|c|c|c|c|c|}
\hline & & \multicolumn{5}{|c|}{ Men } \\
\hline & & \multicolumn{4}{|c|}{ Job Category } & \multirow[b]{2}{*}{ TOTAL } \\
\hline & & Professional & $\begin{array}{c}\text { Administrative } \\
\text { Support }\end{array}$ & $\begin{array}{c}\text { Technical } \\
\text { Support }\end{array}$ & Service & \\
\hline Diagnostic Category & $\begin{array}{l}\text { ICD-9-CM } \\
\text { Code }\end{array}$ & 2 & 0 & 0 & 0 & 2 \\
\hline HEALTH STATUS/HEALTH SERVICE CONTACT & V01-V82 & 0 & 0 & 2 & 0 & 2 \\
\hline -Health Services Reproduction/Development & V20-V29 & 0 & 0 & 2 & 0 & 2 \\
\hline
\end{tabular}

\begin{tabular}{|c|c|c|c|c|c|}
\hline & \multicolumn{5}{|c|}{ Men } \\
\hline & \multicolumn{4}{|c|}{ Job Category } & \multirow[b]{2}{*}{ TOTAL } \\
\hline & Professional & $\begin{array}{c}\text { Administrative } \\
\text { Support }\end{array}$ & \begin{tabular}{|c|} 
Technical \\
Support
\end{tabular} & Service & \\
\hline $\begin{array}{l}\text { Diagnostic } \\
\text { Category }\end{array}$ & & & & & \\
\hline Total & 212 & 9 & 179 & 3 & 403 \\
\hline
\end{tabular}

*Only those diagnostic categories and gender/job category combinations with at least one occurrence appear in this table. 
Los Alamos National Laboratory 2003

Absence Data

Appendix J. Total Number of Calendar Days Absent in Each Diagnostic Category by Gender and Job Category*

\begin{tabular}{|c|c|c|c|c|c|}
\hline & & \multicolumn{4}{|c|}{ Women } \\
\hline & & \multicolumn{3}{|c|}{ Job Category } & \multirow[b]{2}{*}{ TOTAL } \\
\hline & & Professional & $\begin{array}{c}\text { Administrative } \\
\text { Support }\end{array}$ & \begin{tabular}{|l|} 
Technical \\
Support
\end{tabular} & \\
\hline Diagnostic Category & ICD-9-CM Code & \multirow[b]{2}{*}{76} & \multirow[b]{2}{*}{7} & \multirow[b]{2}{*}{26} & \multirow[b]{2}{*}{109} \\
\hline INFECTIOUS \& PARASITIC DISEASES (DIS) & 001-139 & & & & \\
\hline MALIGNANT NEOPLASMS & 140-208, 230-234 & 149 & 0 & 135 & 284 \\
\hline BENIGN \& UNCERTAIN NEOPLASMS & 210-229, 235-239 & 39 & 32 & 0 & 71 \\
\hline ENDOCRINE/METABOLIC/IMMUNITY & $240-279$ & 27 & 9 & 68 & 104 \\
\hline BLOOD \& BLOOD-FORMING ORGANS & 280-289 & 16 & 0 & 0 & 16 \\
\hline MENTAL DISORDERS & $290-319$ & 177 & 4 & 100 & 281 \\
\hline NERVOUS SYSTEM (NS) \& SENSE ORGANS & $320-389$ & 113 & 63 & 26 & 202 \\
\hline CIRCULATORY SYSTEM & $390-459$ & 348 & 0 & 13 & 361 \\
\hline RESPIRATORY SYSTEM & $460-519$ & 270 & 181 & 34 & 485 \\
\hline DIGESTIVE SYSTEM & $520-579$ & 383 & 199 & 97 & 679 \\
\hline GENITOURINARY SYSTEM & $580-629$ & 580 & 246 & 198 & 1,024 \\
\hline PREGNANCY \& CHILDBIRTH & $630-677$ & 20 & 0 & 0 & 20 \\
\hline MUSCULOSKELETAL \& CONNECTIVE TISSUE & 710-739 & 320 & 688 & 292 & 1,300 \\
\hline SYMPTOMS, SIGNS, \& ILL-DEFINED CONDITIONS & 780-799 & 180 & 11 & 37 & 228 \\
\hline INJURY \& POISONING & $800-999$ & 113 & 113 & 114 & 340 \\
\hline
\end{tabular}

\begin{tabular}{|c|c|c|c|c|c|c|}
\hline & & \multicolumn{5}{|c|}{ Men } \\
\hline & & \multicolumn{4}{|c|}{ Job Category } & \multirow[b]{2}{*}{ TOTAL } \\
\hline & & Professional & $\begin{array}{c}\text { Administrative } \\
\text { Support }\end{array}$ & \begin{tabular}{|l|} 
Technical \\
Support
\end{tabular} & Service & \\
\hline Diagnostic Category & ICD-9-CM Code & \multirow[b]{2}{*}{74} & \multirow[b]{2}{*}{0} & \multirow[b]{2}{*}{106} & \multirow[b]{2}{*}{0} & \multirow[b]{2}{*}{180} \\
\hline INFECTIOUS \& PARASITIC DISEASES (DIS) & 001-139 & & & & & \\
\hline MALIGNANT NEOPLASMS & 140-208, 230-234 & 200 & 0 & 1,090 & 0 & 1,290 \\
\hline BENIGN \& UNCERTAIN NEOPLASMS & 210-229, 235-239 & 25 & 0 & 406 & 0 & 431 \\
\hline ENDOCRINE/METABOLIC/IMMUNITY & 240-279 & 38 & 21 & 14 & 0 & 73 \\
\hline MENTAL DISORDERS & 290-319 & 238 & 0 & 1,046 & 0 & 1,284 \\
\hline NERVOUS SYSTEM (NS) \& SENSE ORGANS & 320-389 & 120 & 0 & 49 & 0 & 169 \\
\hline CIRCULATORY SYSTEM & $390-459$ & 990 & 0 & 542 & 0 & 1,532 \\
\hline RESPIRATORY SYSTEM & $460-519$ & 823 & 12 & 392 & 7 & 1,234 \\
\hline DIGESTIVE SYSTEM & $520-579$ & 392 & 84 & 289 & 0 & 765 \\
\hline GENITOURINARY SYSTEM & $580-629$ & 193 & 0 & 29 & 0 & 222 \\
\hline SKIN \& SUBCUTANEOUS TISSUE & 680-709 & 78 & 0 & 0 & 0 & 78 \\
\hline MUSCULOSKELETAL \& CONNECTIVE TISSUE & 710-739 & 669 & 13 & 808 & 0 & 1,490 \\
\hline SYMPTOMS, SIGNS, \& ILL-DEFINED CONDITIONS & 780-799 & 240 & 0 & 114 & 0 & 354 \\
\hline INJURY \& POISONING & 800-999 & 305 & 28 & 468 & 8 & 809 \\
\hline
\end{tabular}

\footnotetext{
*Absences with >1 ICD-9-CM code in the same diagnostic category were counted only once. Only those diagnostic categories and gender/job category combinations with at least one occurrence appear in this table.
} 
Los Alamos National Laboratory 2003

Absence Data

Appendix K. Age-Adjusted Illness and Injury Rates by Diagnostic Category

Part 1. Men

\begin{tabular}{|c|c|c|c|c|c|}
\hline Category of Diagnoses & ICD-9-CM Code & $\begin{array}{l}\text { Number of } \\
\text { Diagnoses }\end{array}$ & $\begin{array}{l}\text { Age-Adjusted } \\
\text { Rate per 1000* }\end{array}$ & \begin{tabular}{|c|} 
Lower $95 \%$ \\
Confidence \\
Limit per 1000
\end{tabular} & $\begin{array}{c}\text { Upper } 95 \% \\
\text { Confidence } \\
\text { Limit per } 1000\end{array}$ \\
\hline Infections \& Parasitic Diseases & $001-139$ & 16 & 1.9 & 1.1 & 3.1 \\
\hline Malignant Neoplasms & $140-208,230-234$ & 7 & 0.7 & 0.3 & 1.5 \\
\hline -Digestive Organs & $150-159$ & 1 & 0.1 & 0.0 & 0.7 \\
\hline -Respiratory System & $160-165$ & 1 & 0.1 & 0.0 & 0.7 \\
\hline -Breast & $174-175$ & 0 & 0 & 0 & 0 \\
\hline -Genitourinary & $179-189$ & 3 & 0.3 & 0.1 & 0.9 \\
\hline -Nervous System & $191-192$ & 0 & 0 & 0 & $\overline{0}$ \\
\hline -Leukemia, Lymphoma & $200-208$ & 0 & 0 & 0 & 0 \\
\hline Benign Neoplasms \& Other & $210-229,235-239$ & 3 & 0.3 & 0.1 & 0.9 \\
\hline Endocrine \& Metabolic Diseases & $240-279$ & 6 & 0.8 & 0.4 & 2.0 \\
\hline Blood \& Blood-Forming Organs & $280-289$ & 0 & 0 & 0 & 0 \\
\hline Mental Disorders & $290-319$ & 11 & 1.5 & 0.8 & 2.8 \\
\hline -Alcoholism & 303 & 1 & 0.2 & 0.0 & 1.3 \\
\hline -Drug Abuse & $304-305$ & 0 & 0 & 0 & 0 \\
\hline Nervous System \& Sense Organs & $320-389$ & 12 & 1.8 & 0.9 & 3.3 \\
\hline Circulatory System & $390-459$ & 32 & 3.7 & 2.6 & 5.4 \\
\hline -Hypertension & 401 & 2 & 0.3 & 0.1 & 1.2 \\
\hline -Acute Myocardial Infarction & 410 & 12 & 1.2 & 0.7 & 2.1 \\
\hline -Ischemic Disease, not M.I. & $411-414,429.2$ & 8 & 0.8 & 0.4 & 1.6 \\
\hline -Cerebrovascular Disease & $430-438$ & 0 & 0 & 0 & 0 \\
\hline Respiratory System & $460-519$ & 105 & 15.1 & 12.0 & 18.9 \\
\hline -Upper Respiratory & $460-465,470-478$ & 55 & 7.7 & 5.7 & 10.5 \\
\hline -Pneumonia/Bronchitis & $466,480-487$ & 34 & 4.7 & 3.2 & 6.9 \\
\hline -Chronic Respiratory Conditions & $490-496$ & 13 & 2.3 & 1.2 & 4.5 \\
\hline Digestive System & $520-579$ & 57 & 7.0 & 5.3 & 9.3 \\
\hline -Hernias & $550-553$ & 28 & 3.5 & 2.4 & 5.2 \\
\hline -Gallbladder Disease & $574-575$ & 6 & 0.6 & 0.3 & 1.3 \\
\hline Genitourinary System & $580-629$ & 8 & 1.1 & 0.5 & 2.3 \\
\hline -Benign Prostatic Hypertrophy & 600 & 0 & 0 & 0 & 0 \\
\hline Skin \& Subcutaneous Tissue & $680-709$ & 3 & 0.6 & 0.2 & 1.9 \\
\hline Musculoskeletal System & $710-739$ & 64 & 9.0 & 6.8 & 11.9 \\
\hline -Dorsopathies & $720-724$ & 17 & 2.3 & 1.4 & 4.0 \\
\hline Congenital Anomalies & $740-759$ & 0 & 0 & 0 & 0 \\
\hline Certain Perinatal Conditions & $760-779$ & 0 & 0 & 0 & 0 \\
\hline Symptoms, Signs, \& Ill Defined Cond & $780-799$ & 23 & 2.5 & 1.6 & 3.8 \\
\hline Injury \& Poisoning & $800-999$ & 54 & 8.4 & 6.2 & 11.4 \\
\hline -Fractures, All Sites & $800-829$ & 14 & 2.1 & 1.2 & 3.7 \\
\hline
\end{tabular}

*Standardized to age distribution of 2000 U.S. population.

**Only women aged 18-45 were included in the calculation of the rates for these diagnostic categories. 
Los Alamos National Laboratory 2003

Absence Data

Appendix K. Age-Adjusted Illness and Injury Rates by Diagnostic Category

\begin{tabular}{|c|c|c|c|c|c|}
\hline Category of Diagnoses & ICD-9-CM Code & $\begin{array}{l}\text { Number of } \\
\text { Diagnoses }\end{array}$ & $\begin{array}{l}\text { Age-Adjusted } \\
\text { Rate per } 1000 *\end{array}$ & \begin{tabular}{|c|} 
Lower $95 \%$ \\
Confidence \\
Limit per 1000
\end{tabular} & $\begin{array}{c}\text { Upper } 95 \% \\
\text { Confidence } \\
\text { Limit per } 1000\end{array}$ \\
\hline -Dislocations & $830-839$ & 0 & 0 & 0 & 0 \\
\hline -Sprains \& Strains & $840-848$ & 14 & 2.1 & 1.2 & 3.9 \\
\hline -Intracranial Injuries & $850-854$ & 0 & 0 & 0 & 0 \\
\hline -Internal Injuries & $860-869$ & 3 & 0.5 & 0.2 & 1.7 \\
\hline -Open Wounds & $870-897$ & 2 & 0.3 & 0.1 & 1.2 \\
\hline -Other Injuries & $900-999$ & 21 & 3.4 & 2.0 & 5.6 \\
\hline Health Status/Health Serv Contact & V01-V82 & 2 & 0.6 & 0.1 & 2.5 \\
\hline -Family History of Health Problems & V10-V19 & 0 & 0 & 0 & 0 \\
\hline -Circumstances Reproduction/Develop & V20-V28 & 2 & 0.6 & 0.1 & 2.5 \\
\hline -Specific Procedure/Aftercare & V50-V59 & 0 & 0 & 0 & 0 \\
\hline Total & & 403 & 54.9 & 49.2 & 61.3 \\
\hline
\end{tabular}

Part 2. Women

\begin{tabular}{|c|c|c|c|c|c|}
\hline Category of Diagnoses & ICD-9-CM Code & $\begin{array}{l}\text { Number of } \\
\text { Diagnoses }\end{array}$ & $\begin{array}{l}\text { Age-Adjusted } \\
\text { Rate per } 1000^{*}\end{array}$ & \begin{tabular}{|c|} 
Lower \\
95\% Confidence \\
Limit per 1000
\end{tabular} & $\begin{array}{c}\text { Upper } \\
\text { 95\% Confidence } \\
\text { Limit per } 1000\end{array}$ \\
\hline Infections \& Parasitic Diseases & $001-139$ & 11 & 2.9 & 1.6 & 5.3 \\
\hline Malignant Neoplasms & $140-208,230-234$ & 9 & 3.0 & 1.4 & 6.7 \\
\hline -Digestive Organs & $150-159$ & 1 & 0.3 & 0.0 & $\overline{1.8}$ \\
\hline -Respiratory System & $160-165$ & 0 & 0 & 0 & 0 \\
\hline -Breast & $174-175$ & 7 & 1.8 & 0.9 & 3.7 \\
\hline -Genitourinary & $179-189$ & 1 & 1.0 & 0.1 & 7.0 \\
\hline -Nervous System & $191-192$ & 0 & 0 & 0 & 0 \\
\hline -Leukemia, Lymphoma & $200-208$ & 0 & 0 & 0 & 0 \\
\hline Benign Neoplasms \& Other & $210-229,235-239$ & 3 & 0.7 & 0.2 & 2.2 \\
\hline Endocrine \& Metabolic Diseases & $240-279$ & 6 & 1.9 & 0.8 & 4.5 \\
\hline Blood \& Blood-Forming Organs & $280-289$ & 1 & 0.3 & 0.0 & 2.4 \\
\hline Mental Disorders & $290-319$ & 12 & 3.1 & 1.7 & 5.7 \\
\hline -Alcoholism & 303 & 0 & 0 & 0 & 0 \\
\hline -Drug Abuse & 304-305 & 0 & 0 & 0 & 0 \\
\hline Nervous System \& Sense Organs & $320-389$ & 14 & 3.2 & 1.9 & 5.5 \\
\hline Circulatory System & $390-459$ & 6 & 2.2 & 0.8 & 6.0 \\
\hline -Hypertension & 401 & 3 & 1.5 & 0.4 & 5.9 \\
\hline -Acute Myocardial Infarction & 410 & 1 & 0.2 & 0.0 & 1.4 \\
\hline -Ischemic Disease, not M.I. & $411-414,429.2$ & 0 & 0 & 0 & 0 \\
\hline -Cerebrovascular Disease & $430-438$ & 0 & 0 & 0 & 0 \\
\hline Respiratory System & $460-519$ & 47 & 11.5 & 8.6 & 15.4 \\
\hline -Upper Respiratory & $460-465,470-478$ & 21 & 5.0 & 3.2 & 7.8 \\
\hline
\end{tabular}

*Standardized to age distribution of 2000 U.S. population.

**Only women aged 18-45 were included in the calculation of the rates for these diagnostic categories. 
Los Alamos National Laboratory 2003

Absence Data

Appendix K. Age-Adjusted Illness and Injury Rates by Diagnostic Category

\begin{tabular}{|c|c|c|c|c|c|}
\hline Category of Diagnoses & ICD-9-CM Code & $\begin{array}{l}\text { Number of } \\
\text { Diagnoses }\end{array}$ & $\begin{array}{l}\text { Age-Adjusted } \\
\text { Rate per } 1000 *\end{array}$ & \begin{tabular}{|c|} 
Lower \\
95\% Confidence \\
Limit per 1000
\end{tabular} & \begin{tabular}{|c|} 
Upper \\
95\% Confidence \\
Limit per 1000
\end{tabular} \\
\hline -Pneumonia/Bronchitis & $466,480-487$ & 19 & 4.8 & 3.1 & 7.7 \\
\hline -Chronic Respiratory Conditions & $490-496$ & 7 & 1.6 & 0.7 & 3.4 \\
\hline Digestive System & $520-579$ & 30 & 7.9 & 5.3 & 11.9 \\
\hline -Hernias & $550-553$ & 1 & 0.3 & 0.0 & 1.8 \\
\hline -Gallbladder Disease & $574-575$ & 18 & 4.5 & 2.8 & 7.2 \\
\hline Genitourinary System & $580-629$ & 34 & 9.2 & 6.4 & 13.2 \\
\hline -Endometriosis & 617 & 0 & 0 & 0 & 0 \\
\hline -Ovarian Cysts & $620.0-620.2$ & 3 & 1.7 & 0.6 & 5.3 \\
\hline -Female Genital Pain/Bleeding & $625-626$ & 2 & 0.5 & 0.1 & 2.2 \\
\hline Pregnancy \& Childbirth ${ }^{* *}$ & $630-639$ & 1 & 0.2 & 0.0 & 1.4 \\
\hline Skin \& Subcutaneous Tissue & $680-709$ & 0 & 0 & 0 & 0 \\
\hline Musculoskeletal System & $710-739$ & 60 & 20.2 & 14.8 & 27.5 \\
\hline -Dorsopathies & $720-724$ & 18 & 4.7 & 2.8 & 7.8 \\
\hline Congenital Anomalies & $740-759$ & 0 & 0 & 0 & 0 \\
\hline Certain Perinatal Conditions & $760-779$ & 0 & 0 & 0 & 0 \\
\hline Symptoms, Signs, \& Ill Defined Cond & $780-799$ & 15 & 3.9 & 2.3 & 6.7 \\
\hline Injury \& Poisoning & $800-999$ & 21 & 6.8 & 4.0 & 11.4 \\
\hline -Fractures, All Sites & $800-829$ & 7 & 2.4 & 0.9 & 6.1 \\
\hline -Dislocations & $830-839$ & 0 & 0 & 0 & 0 \\
\hline -Sprains \& Strains & $840-848$ & 3 & 0.7 & 0.2 & 2.2 \\
\hline -Intracranial Injuries & $850-854$ & 1 & 0.2 & 0.0 & 1.4 \\
\hline -Internal Injuries & $860-869$ & 0 & 0 & 0 & 0 \\
\hline -Open Wounds & $870-897$ & 0 & 0 & 0 & 0 \\
\hline -Other Injuries & $900-999$ & 10 & 3.5 & 1.6 & 7.4 \\
\hline Health Status/Health Serv Contact & V01-V82 & 1 & 0.3 & 0.0 & 2.4 \\
\hline -Family History of Health Problems & V10-V19 & 0 & 0 & 0 & 0 \\
\hline -Circumstances Reproduction/Develop & V20-V28 & 0 & 0 & 0 & 0 \\
\hline -Specific Procedure/Aftercare & V50-V59 & 0 & 0 & 0 & 0 \\
\hline Total & & 271 & 77.4 & 67.4 & 88.8 \\
\hline
\end{tabular}

*Standardized to age distribution of 2000 U.S. population.

**Only women aged 18-45 were included in the calculation of the rates for these diagnostic categories. 
Los Alamos National Laboratory 2003

Absence Data

Appendix K. Age-Adjusted Illness and Injury Rates by Diagnostic Category

Part 3. Men and Women

\begin{tabular}{|c|c|c|c|c|c|}
\hline Category of Diagnoses & ICD-9-CM Code & $\begin{array}{l}\text { Number of } \\
\text { Diagnoses }\end{array}$ & $\begin{array}{l}\text { Age-Adjusted } \\
\text { Rate per } 1000^{*}\end{array}$ & \begin{tabular}{|c|} 
Lower \\
95\% Confidence \\
Limit per 1000
\end{tabular} & $\begin{array}{c}\text { Upper } \\
\text { 95\% Confidence } \\
\text { Limit per } 1000\end{array}$ \\
\hline Infections \& Parasitic Diseases & 001-139 & 27 & 2.2 & 1.5 & 3.2 \\
\hline Malignant Neoplasms & $140-208,230-234$ & 16 & 1.3 & 0.7 & 2.1 \\
\hline -Digestive Organs & $150-159$ & 2 & 0.1 & 0.0 & 0.6 \\
\hline -Respiratory System & $160-165$ & 1 & 0.1 & 0.0 & 0.5 \\
\hline -Breast & $174-175$ & 7 & 0.5 & 0.2 & 1.0 \\
\hline -Genitourinary & $179-189$ & 4 & 0.4 & 0.1 & 1.2 \\
\hline -Nervous System & $191-192$ & 0 & 0 & 0 & 0 \\
\hline -Leukemia, Lymphoma & $200-208$ & 0 & 0 & 0 & 0 \\
\hline Benign Neoplasms \& Other & $210-229,235-239$ & 6 & 0.4 & 0.2 & 0.9 \\
\hline Endocrine \& Metabolic Diseases & $240-279$ & 12 & 1.3 & 0.7 & 2.4 \\
\hline Blood \& Blood-Forming Organs & $280-289$ & 1 & 0.1 & 0.0 & 0.8 \\
\hline Mental Disorders & $290-319$ & 23 & 2.1 & 1.4 & 3.3 \\
\hline -Alcoholism & 303 & 1 & 0.1 & 0.0 & 0.8 \\
\hline -Drug Abuse & $304-305$ & 0 & 0 & 0 & 0 \\
\hline Nervous System \& Sense Organs & $320-389$ & 26 & 2.2 & 1.4 & 3.3 \\
\hline Circulatory System & $390-459$ & 38 & 3.2 & 2.3 & 4.6 \\
\hline -Hypertension & 401 & 5 & 0.5 & 0.2 & 1.4 \\
\hline -Acute Myocardial Infarction & 410 & 13 & 0.9 & 0.5 & 1.6 \\
\hline -Ischemic Disease, not M.I. & $411-414,429.2$ & 8 & 0.6 & 0.3 & 1.1 \\
\hline -Cerebrovascular Disease & $430-438$ & 0 & 0 & 0 & 0 \\
\hline Respiratory System & $460-519$ & 152 & 13.9 & 11.6 & 16.5 \\
\hline -Upper Respiratory & $460-465,470-478$ & 76 & 6.8 & 5.3 & 8.7 \\
\hline -Pneumonia/Bronchitis & $466,480-487$ & 53 & 4.8 & 3.6 & 6.4 \\
\hline -Chronic Respiratory Conditions & $490-496$ & 20 & 2.1 & 1.2 & 3.4 \\
\hline Digestive System & $520-579$ & 87 & 7.3 & 5.8 & 9.2 \\
\hline -Hernias & $550-553$ & 29 & 2.5 & 1.7 & 3.8 \\
\hline -Gallbladder Disease & $574-575$ & 24 & 1.8 & 1.2 & 2.7 \\
\hline Genitourinary System & $580-629$ & 42 & 4.0 & 2.8 & 5.6 \\
\hline -Benign Prostatic Hypertrophy & 600 & 0 & 0 & 0 & 0 \\
\hline -Endometriosis & 617 & 0 & 0 & 0 & 0 \\
\hline -Ovarian Cysts & $620.0-620.2$ & 3 & 0.7 & 0.2 & 2.2 \\
\hline -Female Genital Pain/Bleeding & $625-626$ & 2 & 0.2 & 0.0 & 0.8 \\
\hline Pregnancy \& Childbirth** & $630-639$ & 1 & 0.1 & 0.0 & 0.5 \\
\hline Skin \& Subcutaneous Tissue & $680-709$ & 3 & 0.5 & 0.1 & 1.5 \\
\hline Musculoskeletal System & $710-739$ & 124 & 12.0 & 9.8 & 14.6 \\
\hline -Dorsopathies & $720-724$ & 35 & 3.2 & 2.2 & 4.6 \\
\hline Congenital Anomalies & $740-759$ & 0 & 0 & 0 & 0 \\
\hline
\end{tabular}

*Standardized to age distribution of 2000 U.S. population.

**Only women aged 18-45 were included in the calculation of the rates for these diagnostic categories. 
Los Alamos National Laboratory 2003

Absence Data

Appendix K. Age-Adjusted IIIness and Injury Rates by Diagnostic Category

\begin{tabular}{|c|c|c|c|c|c|}
\hline Category of Diagnoses & ICD-9-CM Code & $\begin{array}{l}\text { Number of } \\
\text { Diagnoses }\end{array}$ & $\begin{array}{l}\text { Age-Adjusted } \\
\text { Rate per } 1000 *\end{array}$ & \begin{tabular}{|c|} 
Lower \\
95\% Confidence \\
Limit per 1000
\end{tabular} & \begin{tabular}{|c|} 
Upper \\
95\% Confidence \\
Limit per 1000
\end{tabular} \\
\hline Certain Perinatal Conditions & $760-779$ & 0 & 0 & 0 & 0 \\
\hline Symptoms, Signs, \& Ill Defined Cond & $780-799$ & 38 & 3.1 & 2.2 & 4.3 \\
\hline Injury \& Poisoning & $800-999$ & 75 & 7.8 & 6.0 & 10.1 \\
\hline -Fractures, All Sites & $800-829$ & 21 & 2.1 & 1.3 & 3.5 \\
\hline -Dislocations & $830-839$ & 0 & 0 & 0 & 0 \\
\hline -Sprains \& Strains & $840-848$ & 17 & 1.7 & 1.0 & 3.0 \\
\hline -Intracranial Injuries & $850-854$ & 1 & 0.1 & 0.0 & 0.5 \\
\hline -Internal Injuries & $860-869$ & 3 & 0.4 & 0.1 & 1.1 \\
\hline -Open Wounds & $870-897$ & 2 & 0.2 & 0.0 & 0.8 \\
\hline -Other Injuries & $900-999$ & 31 & 3.3 & 2.2 & 5.0 \\
\hline Health Status/Health Serv Contact & V01-V82 & 3 & 0.5 & 0.1 & 1.6 \\
\hline -Family History of Health Problems & V10-V19 & 0 & 0 & 0 & 0 \\
\hline -Circumstances Reproduction/Develop & V20-V28 & 2 & 0.4 & 0.1 & 1.5 \\
\hline -Specific Procedure/Aftercare & V50-V59 & 0 & 0 & 0 & 0 \\
\hline Total & & 674 & 61.8 & 56.8 & 67.2 \\
\hline
\end{tabular}

*Standardized to age distribution of 2000 U.S. population.

**Only women aged 18-45 were included in the calculation of the rates for these diagnostic categories. 
Los Alamos National Laboratory 2003

OSHA Data

Appendix L. Number of Workers with at Least One OSHA Event by Gender, Age, and Job Category*

\begin{tabular}{|c|c|c|c|c|c|c|c|c|c|c|c|}
\hline \multirow{3}{*}{ Job Category } & \multicolumn{5}{|c|}{ Women } & \multicolumn{5}{|c|}{ Men } & \multirow[b]{3}{*}{ TOTAL } \\
\hline & \multicolumn{4}{|c|}{ Age Group } & \multirow[b]{2}{*}{ TOTAL } & \multicolumn{4}{|c|}{ Age Group } & \multirow[b]{2}{*}{ TOTAL } & \\
\hline & $16-29$ & 30 - 39 & $40-49$ & $50+$ & & $16-29$ & 30 - 39 & $40-49$ & $50+$ & & \\
\hline Professional & 1 & 0 & 5 & 5 & 11 & 2 & 3 & 10 & 7 & 22 & 33 \\
\hline Administrative Support & 1 & 1 & 3 & 1 & 6 & 0 & 0 & 1 & 0 & 1 & 7 \\
\hline Technical Support & 1 & 0 & 2 & 2 & 5 & 2 & 3 & 6 & 8 & 19 & 24 \\
\hline Service & 0 & 0 & 0 & 0 & 0 & 0 & 0 & 0 & 1 & 1 & 1 \\
\hline TOTAL & 3 & 1 & 10 & 8 & 22 & 4 & 6 & 17 & 16 & 43 & 65 \\
\hline
\end{tabular}

*Only those job categories and gender/age combinations with at least one OSHA event appear in this table.

Appendix M. Total Number of Workdays Lost or with Restricted Activity from OSHA Events by Gender and Age

\begin{tabular}{|c|c|c|c|c|c|c|c|c|c|}
\hline \multirow{2}{*}{$\begin{array}{c}\text { Age } \\
\text { Group }\end{array}$} & \multicolumn{3}{|c|}{ Women } & \multicolumn{3}{|c|}{ Men } & \multicolumn{3}{|c|}{ TOTAL } \\
\hline & $\begin{array}{c}\text { Number } \\
\text { of } \\
\text { Events }\end{array}$ & $\begin{array}{c}\text { Days } \\
\text { Restricted }\end{array}$ & $\begin{array}{l}\text { Days } \\
\text { Lost }\end{array}$ & $\begin{array}{c}\text { Number } \\
\text { of } \\
\text { Events }\end{array}$ & $\begin{array}{c}\text { Days } \\
\text { Restricted }\end{array}$ & $\begin{array}{l}\text { Days } \\
\text { Lost }\end{array}$ & $\begin{array}{c}\text { Number } \\
\text { of } \\
\text { Events }\end{array}$ & $\begin{array}{c}\text { Days } \\
\text { Restricted }\end{array}$ & $\begin{array}{l}\text { Days } \\
\text { Lost }\end{array}$ \\
\hline $16-29$ & 3 & 0 & 0 & 4 & 0 & 0 & 7 & 0 & 0 \\
\hline $30-39$ & 1 & 0 & 0 & 6 & 0 & 0 & 7 & 0 & 0 \\
\hline $40-49$ & 11 & 0 & 0 & 17 & 0 & 0 & 28 & 0 & 0 \\
\hline $50+$ & 8 & 0 & 0 & 16 & 0 & 0 & 24 & 0 & 0 \\
\hline TOTAL & 23 & 0 & 0 & 43 & 0 & 0 & 66 & 0 & 0 \\
\hline
\end{tabular}

Appendix N. Total Number of Workdays Lost or with Restricted Activity from OSHA Events by Gender and Job Category*

\begin{tabular}{|l|r|r|r|r|r|r|r|r|r|}
\hline \multirow{2}{*}{ Job Category } & \multicolumn{3}{|c|}{ Women } & \multicolumn{3}{c|}{ Men } & \multicolumn{3}{c|}{ Total } \\
\cline { 2 - 10 } & $\begin{array}{c}\text { Number } \\
\text { of } \\
\text { Events }\end{array}$ & $\begin{array}{c}\text { Days } \\
\text { Restricted }\end{array}$ & $\begin{array}{c}\text { Days } \\
\text { Lost }\end{array}$ & $\begin{array}{c}\text { Number } \\
\text { of } \\
\text { Events }\end{array}$ & $\begin{array}{c}\text { Days } \\
\text { Restricted }\end{array}$ & $\begin{array}{c}\text { Number } \\
\text { Days } \\
\text { Lost } \\
\text { Events }\end{array}$ & $\begin{array}{c}\text { Days } \\
\text { Restricted }\end{array}$ & $\begin{array}{c}\text { Days } \\
\text { Lost }\end{array}$ \\
\hline Professional & 11 & 0 & 0 & 22 & 0 & 0 & 33 & 0 & 0 \\
\hline Administrative Support & 7 & 0 & 0 & 1 & 0 & 0 & 8 & 0 & 0 \\
\hline Technical Support & 5 & 0 & 0 & 19 & 0 & 0 & 24 & 0 & 0 \\
\hline Service & 0 & 0 & 0 & 1 & 0 & 0 & 1 & 0 & 0 \\
\hline Total & 23 & 0 & 0 & 43 & 0 & 0 & 66 & 0 & 0 \\
\hline
\end{tabular}

*Only those job categories with at least one OSHA event appear in this table. 
Los Alamos National Laboratory 2003

OSHA Data

Appendix O. Number of Diagnoses in Each Diagnostic Category by Gender and Age*

\begin{tabular}{|c|c|c|c|c|c|c|}
\hline & & \multicolumn{5}{|c|}{ Women } \\
\hline & & \multicolumn{4}{|c|}{ Age Group } & \multirow[b]{2}{*}{ TOTAL } \\
\hline & & $16-29$ & 30 - 39 & $40-49$ & $50+$ & \\
\hline Diagnostic Category & ICD-9-CM code & \multirow[b]{2}{*}{0} & \multirow[b]{2}{*}{0} & \multirow[b]{2}{*}{0} & \multirow[b]{2}{*}{0} & \multirow[b]{2}{*}{0} \\
\hline MENTAL DISORDERS & $290-319$ & & & & & \\
\hline -Non-Psychotic Disorders & $300-302,306-316$ & 0 & 0 & 0 & 0 & 0 \\
\hline NERVOUS SYSTEM (NS) \& SENSE ORGANS & 320-389 & 0 & 0 & 1 & 1 & 2 \\
\hline -Disorders of Peripheral NS & $350-359$ & 0 & 0 & 1 & 1 & 2 \\
\hline MUSCULOSKELETAL \& CONNECTIVE TISSUE & $710-739$ & 2 & 0 & 7 & 6 & 15 \\
\hline -Arthropathies & $710-719$ & 2 & 0 & 2 & 3 & 7 \\
\hline -Dorsopathies & $720-724$ & 0 & 0 & 2 & 0 & 2 \\
\hline -Rheumatism, Excluding Back & 725-729 & 0 & 0 & 3 & 3 & 6 \\
\hline SYMPTOMS, SIGNS, \& ILL-DEFINED CONDITIONS & 780-799 & 0 & 0 & 1 & 1 & 2 \\
\hline -Symptoms & 780-789 & 0 & 0 & 1 & 1 & 2 \\
\hline INJURY \& POISONING & $800-999$ & 2 & 1 & 12 & 5 & 20 \\
\hline -Fracture - Upper Limb & 810-819 & 0 & 0 & 0 & 1 & 1 \\
\hline -Sprains \& Strains - Back & 846-847 & 1 & 0 & 3 & 1 & 5 \\
\hline -Sprains \& Strains - Other & $840-845,848$ & 0 & 0 & 8 & 2 & 10 \\
\hline -Open Wound - Head, Neck, Trunk & 870-879 & 0 & 0 & 0 & 1 & 1 \\
\hline -Open Wound - Upper Limb & $880-887$ & 0 & 1 & 1 & 0 & 2 \\
\hline -Open Wound - Lower Limb & $890-897$ & 0 & 0 & 0 & 0 & 0 \\
\hline -Superficial Injury & 910-919 & 0 & 0 & 0 & 0 & 0 \\
\hline -Contusion & $920-924$ & 0 & 0 & 0 & 0 & 0 \\
\hline -Foreign Body Entering Orifice & $930-939$ & 0 & 0 & 0 & 0 & 0 \\
\hline -Burns & 940-949 & 1 & 0 & 0 & 0 & 1 \\
\hline -Complications \& Unspecified Injuries & 958-959 & 0 & 0 & 0 & 0 & 0 \\
\hline -Toxic Effects - Non-medicinal & \begin{tabular}{|c|}
$980-989$ \\
\end{tabular} & 0 & 0 & 0 & 0 & 0 \\
\hline
\end{tabular}

*Only those diagnostic categories and gender/age combinations with at least one OSHA event appear in this table. 
Los Alamos National Laboratory 2003

OSHA Data

Appendix O. Number of Diagnoses in Each Diagnostic Category by Gender and Age*

\begin{tabular}{|c|c|c|c|c|c|c|c|}
\hline & & \multicolumn{5}{|c|}{ Men } & \multirow[b]{3}{*}{ TOTAL } \\
\hline & & \multicolumn{4}{|c|}{ Age Group } & \multirow[b]{2}{*}{ TOTAL } & \\
\hline & & $16-29$ & 30 - 39 & $40-49$ & $\mathbf{5 0 +}$ & & \\
\hline Diagnostic Category & ICD-9-CM code & & & & & & \\
\hline MENTAL DISORDERS & 290-319 & 0 & 0 & 0 & 1 & 1 & 1 \\
\hline -Non-Psychotic Disorders & $300-302,306-316$ & 0 & 0 & 0 & 1 & 1 & 1 \\
\hline NERVOUS SYSTEM (NS) \& SENSE ORGANS & $320-389$ & 0 & 0 & 0 & 0 & 0 & 2 \\
\hline -Disorders of Peripheral NS & $350-359$ & 0 & 0 & 0 & 0 & 0 & 2 \\
\hline MUSCULOSKELETAL \& CONNECTIVE TISSUE & 710-739 & 2 & 2 & 5 & 2 & 11 & 26 \\
\hline -Arthropathies & 710-719 & 0 & 2 & 3 & 1 & 6 & 13 \\
\hline -Dorsopathies & $720-724$ & 1 & 0 & 0 & 0 & 1 & 3 \\
\hline -Rheumatism, Excluding Back & $725-729$ & 1 & 0 & 2 & 1 & 4 & 10 \\
\hline SYMPTOMS, SIGNS, \& ILL-DEFINED CONDITIONS & 780-799 & 0 & 0 & 1 & 0 & 1 & 3 \\
\hline -Symptoms & 780-789 & 0 & 0 & 1 & 0 & 1 & 3 \\
\hline INJURY \& POISONING & 800-999 & 3 & 4 & 13 & 15 & 35 & 55 \\
\hline -Fracture - Upper Limb & 810-819 & 0 & 0 & 0 & 0 & 0 & 1 \\
\hline -Sprains \& Strains - Back & 846-847 & 0 & 0 & 2 & 2 & 4 & 9 \\
\hline -Sprains \& Strains - Other & $840-845,848$ & 1 & 1 & 3 & 1 & 6 & 16 \\
\hline -Open Wound - Head, Neck, Trunk & 870-879 & 0 & 0 & 2 & 1 & 3 & 4 \\
\hline -Open Wound - Upper Limb & 880-887 & 0 & 1 & 3 & 3 & 7 & 9 \\
\hline -Open Wound - Lower Limb & 890-897 & 0 & 0 & 0 & 1 & 1 & 1 \\
\hline -Superficial Injury & 910-919 & 0 & 0 & 0 & 3 & 3 & 3 \\
\hline -Contusion & $920-924$ & 0 & 0 & 0 & 2 & 2 & 2 \\
\hline -Foreign Body Entering Orifice & 930-939 & 1 & 1 & 0 & 0 & 2 & 2 \\
\hline -Burns & 940-949 & 0 & 1 & 1 & 0 & 2 & 3 \\
\hline -Complications \& Unspecified Injuries & 958-959 & 0 & 0 & 0 & 2 & 2 & 2 \\
\hline -Toxic Effects - Non-medicinal & 980-989 & 1 & 0 & 2 & 0 & 3 & 3 \\
\hline
\end{tabular}

\begin{tabular}{|c|c|c|c|c|c|c|c|c|c|c|c|}
\hline & \multicolumn{5}{|c|}{ Women } & \multicolumn{5}{|c|}{ Men } & \multirow[b]{3}{*}{ TOTAL } \\
\hline & \multicolumn{4}{|c|}{ Age Group } & \multirow[b]{2}{*}{ TOTAL } & \multicolumn{4}{|c|}{ Age Group } & \multirow[b]{2}{*}{ TOTAL } & \\
\hline & $16-29$ & 30 - 39 & $40-49$ & $50+$ & & $16-29$ & 30 - 39 & $40-49$ & $50+$ & & \\
\hline $\begin{array}{l}\text { Diagnostic } \\
\text { Category }\end{array}$ & & & & & & & & & & & \\
\hline Total & 4 & 1 & 21 & 13 & 39 & 5 & 6 & 19 & 18 & 48 & 87 \\
\hline
\end{tabular}

*Only those diagnostic categories and gender/age combinations with at least one OSHA event appear in this table. 
Los Alamos National Laboratory 2003

OSHA Data

Appendix P. Number of Workdays Lost or with Restricted Activity in Each Diagnostic Category by Gender and Age*

\begin{tabular}{|c|c|c|c|c|c|c|c|c|c|}
\hline & & \multicolumn{8}{|c|}{ Women } \\
\hline & & \multicolumn{8}{|c|}{ Age Group } \\
\hline & & \multicolumn{2}{|c|}{$16-29$} & \multicolumn{2}{|c|}{$30-39$} & \multicolumn{2}{|c|}{$40-49$} & \multicolumn{2}{|l|}{$50+$} \\
\hline & & $\begin{array}{c}\text { Days } \\
\text { Restricted }\end{array}$ & \begin{tabular}{|l|} 
Days \\
Lost
\end{tabular} & \begin{tabular}{|c|} 
Days \\
Restricted
\end{tabular} & $\begin{array}{l}\text { Days } \\
\text { Lost }\end{array}$ & $\begin{array}{c}\text { Days } \\
\text { Restricted }\end{array}$ & \begin{tabular}{|l|} 
Days \\
Lost
\end{tabular} & $\begin{array}{c}\text { Days } \\
\text { Restricted }\end{array}$ & \begin{tabular}{|l} 
Days \\
Lost
\end{tabular} \\
\hline Diagnostic Category & $\begin{array}{l}\text { ICD-9-CM } \\
\text { Codes }\end{array}$ & & & & & & & & \\
\hline -Disorders of Peripheral NS & $350-359$ & 0 & 0 & 0 & 0 & 0 & 0 & 0 & 0 \\
\hline -Arthropathies & $710-719$ & 0 & 0 & 0 & 0 & 0 & 0 & 0 & 0 \\
\hline -Dorsopathies & $720-724$ & 0 & 0 & 0 & 0 & 0 & 0 & 0 & 0 \\
\hline -Rheumatism, Excluding Back & $725-729$ & 0 & 0 & 0 & 0 & 0 & 0 & 0 & 0 \\
\hline -Symptoms & 780-789 & 0 & 0 & 0 & 0 & 0 & 0 & 0 & 0 \\
\hline -Fracture - Upper Limb & $810-819$ & 0 & 0 & 0 & 0 & 0 & 0 & 0 & 0 \\
\hline -Sprains \& Strains - Back & 846-847 & 0 & 0 & 0 & 0 & 0 & 0 & 0 & 0 \\
\hline -Sprains \& Strains - Other & $840-845,848$ & 0 & 0 & 0 & 0 & 0 & 0 & 0 & 0 \\
\hline -Open Wound - Head, Neck, Trunk & $870-879$ & 0 & 0 & 0 & 0 & 0 & 0 & 0 & 0 \\
\hline -Open Wound - Upper Limb & $880-887$ & 0 & 0 & 0 & 0 & 0 & 0 & 0 & 0 \\
\hline -Burns & 940-949 & 0 & 0 & 0 & 0 & 0 & 0 & 0 & 0 \\
\hline
\end{tabular}

\begin{tabular}{|c|c|c|c|c|c|c|c|c|c|}
\hline & & \multicolumn{8}{|c|}{ Men } \\
\hline & & \multicolumn{8}{|c|}{ Age Group } \\
\hline & & \multicolumn{2}{|c|}{$16-29$} & \multicolumn{2}{|c|}{$30-39$} & \multicolumn{2}{|c|}{$40-49$} & \multicolumn{2}{|l|}{$50+$} \\
\hline Diagnostic Category & $\begin{array}{l}\text { ICD-9-CM } \\
\text { Codes }\end{array}$ & & & & & & & & \\
\hline -Arthropathies & $710-719$ & 0 & 0 & 0 & 0 & 0 & 0 & 0 & 0 \\
\hline -Dorsopathies & $720-724$ & 0 & 0 & 0 & 0 & 0 & 0 & 0 & 0 \\
\hline -Rheumatism, Excluding Back & 725-729 & 0 & 0 & 0 & 0 & 0 & 0 & 0 & 0 \\
\hline -Symptoms & $780-789$ & 0 & 0 & 0 & 0 & 0 & 0 & 0 & 0 \\
\hline -Open Wound - Head, Neck, Trunk & $870-879$ & 0 & 0 & 0 & 0 & 0 & 0 & 0 & 0 \\
\hline -Open Wound - Upper Limb & $880-887$ & 0 & 0 & 0 & 0 & 0 & 0 & 0 & 0 \\
\hline -Open Wound - Lower Limb & $890-897$ & 0 & 0 & 0 & 0 & 0 & 0 & 0 & 0 \\
\hline -Superficial Injury & $910-919$ & 0 & 0 & 0 & 0 & 0 & 0 & 0 & 0 \\
\hline -Contusion & $920-924$ & 0 & 0 & 0 & 0 & 0 & 0 & 0 & 0 \\
\hline -Foreign Body Entering Orifice & $930-939$ & 0 & 0 & 0 & 0 & 0 & 0 & 0 & 0 \\
\hline -Burns & $940-949$ & 0 & 0 & 0 & 0 & 0 & 0 & 0 & 0 \\
\hline -Complications \& Unspecified Injuries & 958-959 & 0 & 0 & 0 & 0 & 0 & 0 & 0 & 0 \\
\hline -Toxic Effects - Non-medicinal & 980-989 & 0 & 0 & 0 & 0 & 0 & 0 & 0 & 0 \\
\hline
\end{tabular}

*OSHA events with >1 ICD-9-CM code in the same diagnostic category were counted only once. Only those diagnostic categories and gender/age combinations with at least one occurrence appear in this table. 
Los Alamos National Laboratory 2003

OSHA Data

Appendix Q. Number of Occurrences in Each Accident Category by Gender and Age*

\begin{tabular}{|c|c|c|c|c|c|c|c|c|c|}
\hline & & \multicolumn{2}{|c|}{ Women } & \multicolumn{5}{|c|}{ Men } & \multirow[b]{3}{*}{ TOTAL } \\
\hline & & \multirow{2}{*}{$\begin{array}{c}\begin{array}{c}\text { Age } \\
\text { Group }\end{array} \\
16-29\end{array}$} & \multirow[b]{2}{*}{ TOTAL } & \multicolumn{4}{|c|}{ Age Group } & \multirow[b]{2}{*}{ TOTAL } & \\
\hline & & & & $16-29$ & 30 - 39 & $40-49$ & $50+$ & & \\
\hline Type of Accident & E Codes & & & & & & & & \\
\hline Motor Vehicle Traffic & E810-E819 & 0 & 0 & 1 & 0 & 0 & 0 & 1 & 1 \\
\hline Accidental Poisoning - Non-medicinal & E860-E869 & 0 & 0 & 1 & 0 & 2 & 0 & 3 & 3 \\
\hline Natural/Environmental Factors & E900-E909 & 0 & 0 & 0 & 0 & 0 & 1 & 1 & 1 \\
\hline Submersion/Suffocation/Foreign Bodies & E910-E915 & 0 & 0 & 1 & 0 & 0 & 0 & 1 & 1 \\
\hline Other Accidents & E916-E928 & 1 & 1 & 0 & 1 & 0 & 1 & 2 & 3 \\
\hline
\end{tabular}

*Only those accident types and gender/age combinations with at least one occurrence appear in this table.

Appendix R. Number of Workdays Lost or with Restricted Activity in Each Accident Category by Gender and Age*

\begin{tabular}{|l|l|r|r|}
\hline \multicolumn{2}{|c|}{} & \multicolumn{2}{|c|}{ Women } \\
\cline { 3 - 4 } & \multicolumn{2}{|c|}{ Age Group } \\
\cline { 3 - 4 } & \multicolumn{2}{|c|}{16 - 29 } \\
\cline { 3 - 4 } \multicolumn{2}{|c|}{} & $\begin{array}{c}\text { Days } \\
\text { Restricted }\end{array}$ & $\begin{array}{r}\text { Days } \\
\text { Lost }\end{array}$ \\
\hline $\begin{array}{l}\text { Type of } \\
\text { Accident }\end{array}$ & E Codes & & \\
\hline Other Accidents & E916-E928 & 0 & 0 \\
\hline
\end{tabular}

\begin{tabular}{|c|c|c|c|c|c|c|c|c|c|}
\hline & \multicolumn{8}{|c|}{ Men } \\
\hline & & \multicolumn{8}{|c|}{ Age Group } \\
\hline & & \multicolumn{2}{|c|}{$16-29$} & \multicolumn{2}{|c|}{$30-39$} & \multicolumn{2}{|c|}{$40-49$} & \multicolumn{2}{|l|}{$50+$} \\
\hline & & $\begin{array}{c}\text { Days } \\
\text { Restricted }\end{array}$ & \begin{tabular}{|l|} 
Days \\
Lost
\end{tabular} & \begin{tabular}{|c|} 
Days \\
Restricted
\end{tabular} & \begin{tabular}{|l|} 
Days \\
Lost \\
\end{tabular} & \begin{tabular}{|c|} 
Days \\
Restricted
\end{tabular} & \begin{tabular}{|l|} 
Days \\
Lost \\
\end{tabular} & \begin{tabular}{c|c} 
Days \\
Restricted
\end{tabular} & $\begin{array}{l}\text { Days } \\
\text { Lost }\end{array}$ \\
\hline Type of Accident & E Codes & & & & & & & & \\
\hline Motor Vehicle Traffic & E810-E819 & 0 & 0 & 0 & 0 & 0 & 0 & 0 & 0 \\
\hline Accidental Poisoning - Non-medicinal & E860-E869 & 0 & 0 & 0 & 0 & 0 & 0 & 0 & 0 \\
\hline Natural/Environmental Factors & E900-E909 & 0 & 0 & 0 & 0 & 0 & 0 & 0 & 0 \\
\hline Submersion/Suffocation/Foreign Bodies & E910-E915 & 0 & 0 & 0 & 0 & 0 & 0 & 0 & 0 \\
\hline Other Accidents & E916-E928 & 0 & 0 & 0 & 0 & 0 & 0 & 0 & 0 \\
\hline
\end{tabular}

*OSHA events with $>1 \mathrm{E}$ code in the same accident type were counted only once. Only those accident types and gender/age combinations with at least one occurrence appear in this table. 
Los Alamos National Laboratory 2003

OSHA Data

Appendix S. Number of Diagnoses in Each Diagnostic Category by Gender and Job Category*

\begin{tabular}{|c|c|c|c|c|c|}
\hline & & \multicolumn{4}{|c|}{ Women } \\
\hline & & \multicolumn{3}{|c|}{ Job Category } & \multirow[b]{2}{*}{ TOTAL } \\
\hline & & Professional & $\begin{array}{c}\text { Administrative } \\
\text { Support }\end{array}$ & $\begin{array}{l}\text { Technical } \\
\text { Support }\end{array}$ & \\
\hline Diagnostic Category & $\begin{array}{l}\text { ICD-9-CM } \\
\text { Code }\end{array}$ & \multirow[b]{2}{*}{2} & \multirow[b]{2}{*}{0} & \multirow[b]{2}{*}{0} & \multirow[b]{2}{*}{2} \\
\hline NERVOUS SYSTEM (NS) \& SENSE ORGANS & 320-389 & & & & \\
\hline -Disorders of Peripheral NS & $350-359$ & 2 & 0 & 0 & 2 \\
\hline MUSCULOSKELETAL \& CONNECTIVE TISSUE & 710-739 & 8 & 6 & 1 & 15 \\
\hline -Arthropathies & 710-719 & 1 & 5 & 1 & 7 \\
\hline -Dorsopathies & $720-724$ & 2 & 0 & 0 & 2 \\
\hline -Rheumatism, Excluding Back & 725-729 & 5 & 1 & 0 & 6 \\
\hline SYMPTOMS, SIGNS, \& ILL-DEFINED CONDITIONS & 780-799 & 2 & 0 & 0 & 2 \\
\hline -Symptoms & $780-789$ & 2 & 0 & 0 & 2 \\
\hline INJURY \& POISONING & $800-999$ & 8 & 5 & 7 & 20 \\
\hline -Fracture - Upper Limb & 810-819 & 1 & 0 & 0 & 1 \\
\hline -Sprains \& Strains - Back & 846-847 & 4 & 0 & 1 & 5 \\
\hline -Sprains \& Strains - Other & 840-845, 848 & 3 & 3 & 4 & 10 \\
\hline -Open Wound - Head, Neck, Trunk & $870-879$ & 0 & 0 & 1 & 1 \\
\hline -Open Wound - Upper Limb & $880-887$ & 0 & 2 & 0 & 2 \\
\hline -Burns & $940-949$ & 0 & 0 & 1 & 1 \\
\hline
\end{tabular}

\begin{tabular}{|c|c|c|c|c|}
\hline & \multicolumn{4}{|c|}{ Women } \\
\hline & \multicolumn{3}{|c|}{ Job Category } & \multirow[b]{2}{*}{ TOTAL } \\
\hline & Professional & $\begin{array}{l}\text { Administrative } \\
\text { Support }\end{array}$ & $\begin{array}{l}\text { Technical } \\
\text { Support }\end{array}$ & \\
\hline $\begin{array}{l}\text { Diagnostic } \\
\text { Category }\end{array}$ & \multirow[b]{2}{*}{20} & \multirow[b]{2}{*}{11} & \multirow[b]{2}{*}{8} & \multirow[b]{2}{*}{39} \\
\hline Total & & & & \\
\hline
\end{tabular}

\begin{tabular}{|c|c|c|c|c|c|c|}
\hline & & \multicolumn{5}{|c|}{ Men } \\
\hline & & \multicolumn{4}{|c|}{ Job Category } & \multirow[b]{2}{*}{ TOTAL } \\
\hline & & Professional & $\begin{array}{c}\text { Administrative } \\
\text { Support }\end{array}$ & \begin{tabular}{|l|} 
Technical \\
Support
\end{tabular} & Service & \\
\hline Diagnostic Category & ICD-9-CM Code & \multirow[b]{2}{*}{1} & \multirow[b]{2}{*}{0} & \multirow[b]{2}{*}{0} & \multirow[b]{2}{*}{0} & \multirow[b]{2}{*}{1} \\
\hline MENTAL DISORDERS & 290-319 & & & & & \\
\hline -Non-Psychotic Disorders & $300-302,306-316$ & 1 & 0 & 0 & 0 & 1 \\
\hline MUSCULOSKELETAL \& CONNECTIVE TISSUE & 710-739 & 4 & 1 & 6 & 0 & 11 \\
\hline -Arthropathies & $710-719$ & 1 & 0 & 5 & 0 & 6 \\
\hline -Dorsopathies & $720-724$ & 0 & 0 & 1 & 0 & 1 \\
\hline -Rheumatism, Excluding Back & 725-729 & 3 & 1 & 0 & 0 & 4 \\
\hline SYMPTOMS, SIGNS, \& ILL-DEFINED CONDITIONS & 780-799 & 0 & 0 & 1 & 0 & 1 \\
\hline -Symptoms & 780-789 & 0 & 0 & 1 & 0 & 1 \\
\hline
\end{tabular}

(Continued)

*Only those diagnostic categories and gender/job category combinations with at least one occurrence appear in this table. 
Los Alamos National Laboratory 2003

OSHA Data

Appendix S. Number of Diagnoses in Each Diagnostic Category by Gender and Job Category*

\begin{tabular}{|c|c|c|c|c|c|c|}
\hline & & \multicolumn{5}{|c|}{ Men } \\
\hline & & \multicolumn{4}{|c|}{ Job Category } & \multirow[b]{2}{*}{ TOTAL } \\
\hline & & Professional & $\begin{array}{c}\text { Administrative } \\
\text { Support }\end{array}$ & \begin{tabular}{|l|} 
Technical \\
Support
\end{tabular} & Service & \\
\hline Diagnostic Category & ICD-9-CM Code & \multirow[b]{2}{*}{20} & \multirow[b]{2}{*}{0} & \multirow[b]{2}{*}{14} & \multirow[b]{2}{*}{1} & \multirow[b]{2}{*}{35} \\
\hline INJURY \& POISONING & $800-999$ & & & & & \\
\hline -Sprains \& Strains - Back & 846-847 & 4 & 0 & 0 & 0 & 4 \\
\hline -Sprains \& Strains - Other & $840-845,848$ & 5 & 0 & 1 & 0 & 6 \\
\hline -Open Wound - Head, Neck, Trunk & 870-879 & 3 & 0 & 0 & 0 & 3 \\
\hline -Open Wound - Upper Limb & 880-887 & 1 & 0 & 6 & 0 & 7 \\
\hline -Open Wound - Lower Limb & 890-897 & 0 & 0 & 1 & 0 & 1 \\
\hline -Superficial Injury & 910-919 & 1 & 0 & 1 & 1 & 3 \\
\hline -Contusion & 920-924 & 2 & 0 & 0 & 0 & 2 \\
\hline -Foreign Body Entering Orifice & 930-939 & 2 & 0 & 0 & 0 & 2 \\
\hline -Burns & 940-949 & 1 & 0 & 1 & 0 & 2 \\
\hline -Complications \& Unspecified Injuries & 958-959 & 1 & 0 & 1 & 0 & 2 \\
\hline -Toxic Effects - Non-medicinal & 980-989 & 0 & 0 & 3 & 0 & 3 \\
\hline
\end{tabular}

\begin{tabular}{|c|c|c|c|c|c|}
\hline & \multicolumn{5}{|c|}{ Men } \\
\hline & \multicolumn{4}{|c|}{ Job Category } & \multirow[b]{2}{*}{ TOTAL } \\
\hline & Professional & $\begin{array}{l}\text { Administrative } \\
\text { Support }\end{array}$ & $\begin{array}{l}\text { Technical } \\
\text { Support }\end{array}$ & Service & \\
\hline $\begin{array}{l}\text { Diagnostic } \\
\text { Category }\end{array}$ & \multirow[b]{2}{*}{25} & \multirow[b]{2}{*}{1} & \multirow[b]{2}{*}{21} & \multirow[b]{2}{*}{1} & \multirow[b]{2}{*}{48} \\
\hline Total & & & & & \\
\hline
\end{tabular}

*Only those diagnostic categories and gender/job category combinations with at least one occurrence appear in this table. 


\section{Los Alamos National Laboratory 2003}

\section{OSHA Data}

Appendix T. Number of Workdays Lost or with Restricted Activity in Each Diagnostic Category by Gender and Job Category*

\begin{tabular}{|c|c|c|c|c|c|c|c|}
\hline & & \multicolumn{6}{|c|}{ Women } \\
\hline & & \multicolumn{6}{|c|}{ Job Category } \\
\hline & & \multicolumn{2}{|c|}{ Professional } & \multicolumn{2}{|c|}{$\begin{array}{l}\text { Administrative } \\
\text { Support }\end{array}$} & \multicolumn{2}{|c|}{$\begin{array}{l}\text { Technical } \\
\text { Support }\end{array}$} \\
\hline & & $\begin{array}{c}\text { Days } \\
\text { Restricted }\end{array}$ & $\begin{array}{l}\text { Days } \\
\text { Lost }\end{array}$ & $\begin{array}{c}\text { Days } \\
\text { Restricted }\end{array}$ & $\begin{array}{l}\text { Days } \\
\text { Lost }\end{array}$ & $\begin{array}{c}\text { Days } \\
\text { Restricted }\end{array}$ & $\begin{array}{l}\text { Days } \\
\text { Lost }\end{array}$ \\
\hline Diagnostic Category & $\begin{array}{l}\text { ICD-9-CM } \\
\text { Codes }\end{array}$ & \multirow[b]{2}{*}{0} & \multirow[b]{2}{*}{0} & \multirow[b]{2}{*}{0} & \multirow[b]{2}{*}{0} & \multirow[b]{2}{*}{0} & \multirow[b]{2}{*}{0} \\
\hline -Disorders of Peripheral NS & $350-359$ & & & & & & \\
\hline -Arthropathies & \begin{tabular}{|l|}
$710-719$ \\
\end{tabular} & 0 & 0 & 0 & 0 & 0 & 0 \\
\hline -Dorsopathies & $720-724$ & 0 & 0 & 0 & 0 & 0 & 0 \\
\hline -Rheumatism, Excluding Back & \begin{tabular}{|l|}
$725-729$ \\
\end{tabular} & 0 & 0 & 0 & 0 & 0 & $\overline{0}$ \\
\hline -Symptoms & 780-789 & 0 & 0 & 0 & 0 & 0 & 0 \\
\hline -Fracture - Upper Limb & $810-819$ & 0 & 0 & 0 & 0 & 0 & 0 \\
\hline -Sprains \& Strains - Back & 846-847 & 0 & 0 & 0 & 0 & 0 & 0 \\
\hline -Sprains \& Strains - Other & $840-845,848$ & 0 & 0 & 0 & 0 & 0 & 0 \\
\hline -Open Wound - Head, Neck, Trunk & $870-879$ & 0 & 0 & 0 & 0 & 0 & 0 \\
\hline -Open Wound - Upper Limb & $880-887$ & 0 & 0 & 0 & 0 & 0 & 0 \\
\hline -Burns & \begin{tabular}{|c|}
$940-949$ \\
\end{tabular} & 0 & 0 & 0 & 0 & 0 & 0 \\
\hline
\end{tabular}

\begin{tabular}{|c|c|c|c|c|c|c|c|c|c|}
\hline & & \multicolumn{8}{|c|}{ Men } \\
\hline & & \multicolumn{8}{|c|}{ Job Category } \\
\hline & & \multicolumn{2}{|c|}{ Professional } & \multicolumn{2}{|c|}{$\begin{array}{c}\text { Administrative } \\
\text { Support }\end{array}$} & \multicolumn{2}{|c|}{$\begin{array}{l}\text { Technical } \\
\text { Support }\end{array}$} & \multicolumn{2}{|c|}{ Service } \\
\hline & & $\begin{array}{c}\text { Days } \\
\text { Restricted }\end{array}$ & $\begin{array}{l}\text { Days } \\
\text { Lost }\end{array}$ & \begin{tabular}{c|} 
Days \\
Restricted
\end{tabular} & $\begin{array}{l}\text { Days } \\
\text { Lost }\end{array}$ & \begin{tabular}{|c|} 
Days \\
Restricted
\end{tabular} & $\begin{array}{l}\text { Days } \\
\text { Lost }\end{array}$ & $\begin{array}{c}\text { Days } \\
\text { Restricted }\end{array}$ & \begin{tabular}{|l} 
Days \\
Lost
\end{tabular} \\
\hline Diagnostic Category & $\begin{array}{l}\text { ICD-9-CM } \\
\text { Codes }\end{array}$ & & & & & & & & \\
\hline -Non-Psychotic Disorders & $300-302,306-316$ & 0 & 0 & 0 & 0 & 0 & 0 & 0 & 0 \\
\hline -Arthropathies & $710-719$ & 0 & 0 & 0 & 0 & 0 & 0 & 0 & 0 \\
\hline -Dorsopathies & $720-724$ & 0 & 0 & 0 & 0 & 0 & 0 & 0 & 0 \\
\hline -Rheumatism, Excluding Back & $725-729$ & 0 & 0 & 0 & 0 & 0 & 0 & 0 & 0 \\
\hline -Symptoms & $780-789$ & 0 & 0 & 0 & 0 & 0 & 0 & 0 & 0 \\
\hline -Sprains \& Strains - Back & 846-847 & 0 & 0 & 0 & 0 & 0 & 0 & 0 & 0 \\
\hline -Sprains \& Strains - Other & $840-845,848$ & 0 & 0 & 0 & 0 & 0 & 0 & 0 & 0 \\
\hline -Open Wound - Head, Neck, Trunk & 870-879 & 0 & 0 & 0 & 0 & 0 & 0 & 0 & 0 \\
\hline -Open Wound - Upper Limb & \begin{tabular}{|c|}
$880-887$ \\
\end{tabular} & 0 & 0 & 0 & 0 & 0 & 0 & 0 & 0 \\
\hline -Open Wound - Lower Limb & $890-897$ & 0 & 0 & 0 & 0 & 0 & 0 & 0 & 0 \\
\hline -Superficial Injury & 910-919 & 0 & 0 & 0 & 0 & 0 & 0 & 0 & 0 \\
\hline -Contusion & $920-924$ & 0 & 0 & 0 & 0 & 0 & 0 & 0 & 0 \\
\hline -Foreign Body Entering Orifice & $930-939$ & 0 & 0 & 0 & 0 & 0 & 0 & 0 & 0 \\
\hline -Burns & 940-949 & 0 & 0 & 0 & 0 & 0 & 0 & 0 & 0 \\
\hline
\end{tabular}

(Continued)

*OSHA events with >1 ICD-9-CM code in the same diagnostic category were counted only once. Only those diagnostic categories and gender/job category combinations with at least one occurrence appear in this table. 
Los Alamos National Laboratory 2003

OSHA Data

Appendix T. Number of Workdays Lost or with Restricted Activity in Each Diagnostic Category by Gender and Job Category*

\begin{tabular}{|c|c|c|c|c|c|c|c|c|c|}
\hline & & \multicolumn{8}{|c|}{ Men } \\
\hline & & \multicolumn{8}{|c|}{ Job Category } \\
\hline & & \multicolumn{2}{|c|}{ Professional } & \multicolumn{2}{|c|}{$\begin{array}{l}\text { Administrative } \\
\text { Support }\end{array}$} & \multicolumn{2}{|c|}{$\begin{array}{l}\text { Technical } \\
\text { Support }\end{array}$} & \multicolumn{2}{|c|}{ Service } \\
\hline & & $\begin{array}{c}\text { Days } \\
\text { Restricted }\end{array}$ & $\begin{array}{l}\text { Days } \\
\text { Lost }\end{array}$ & \begin{tabular}{c|} 
Days \\
Restricted
\end{tabular} & $\begin{array}{l}\text { Days } \\
\text { Lost }\end{array}$ & $\begin{array}{c}\text { Days } \\
\text { Restricted }\end{array}$ & $\begin{array}{l}\text { Days } \\
\text { Lost }\end{array}$ & $\begin{array}{c}\text { Days } \\
\text { Restricted }\end{array}$ & $\begin{array}{l}\text { Days } \\
\text { Lost }\end{array}$ \\
\hline Diagnostic Category & $\begin{array}{l}\text { ICD-9-CM } \\
\text { Codes }\end{array}$ & & & & & & & & \\
\hline -Complications \& Unspecified Injuries & $958-959$ & 0 & 0 & 0 & 0 & 0 & 0 & 0 & 0 \\
\hline -Toxic Effects - Non-medicinal & $980-989$ & 0 & 0 & 0 & 0 & 0 & 0 & 0 & 0 \\
\hline
\end{tabular}

*OSHA events with >1 ICD-9-CM code in the same diagnostic category were counted only once. Only those diagnostic categories and gender/job category combinations with at least one occurrence appear in this table. 
Los Alamos National Laboratory 2003

OSHA Data

Appendix U. Number of Occurrences in Each Accident Category by Gender and Job Category*

\begin{tabular}{|c|c|c|c|}
\hline & & \multicolumn{2}{|c|}{ Women } \\
\hline & & $\begin{array}{c}\text { Job } \\
\text { Category }\end{array}$ & \multirow[b]{2}{*}{ TOTAL } \\
\hline & & $\begin{array}{l}\text { Technical } \\
\text { Support }\end{array}$ & \\
\hline $\begin{array}{l}\text { Type of } \\
\text { Accident }\end{array}$ & E CODES & \multirow[b]{2}{*}{1} & \multirow[b]{2}{*}{1} \\
\hline Other Accidents & E916-E928 & & \\
\hline
\end{tabular}

\begin{tabular}{|c|c|c|c|c|}
\hline & & \multicolumn{3}{|c|}{ Men } \\
\hline & & \multicolumn{2}{|c|}{ Job Category } & \multirow[b]{2}{*}{ TOTAL } \\
\hline & & Professional & $\begin{array}{l}\text { Technical } \\
\text { Support }\end{array}$ & \\
\hline Type of Accident & E CODES & \multirow[b]{2}{*}{0} & \multirow[b]{2}{*}{1} & \multirow[b]{2}{*}{1} \\
\hline Motor Vehicle Traffic & E810-E819 & & & \\
\hline Accidental Poisoning - Non-medicinal & E860-E869 & 0 & 3 & 3 \\
\hline Natural/Environmental Factors & E900-E909 & 1 & 0 & 1 \\
\hline Submersion/Suffocation/Foreign Bodies & E910-E915 & 1 & 0 & 1 \\
\hline Other Accidents & E916-E928 & 2 & 0 & 2 \\
\hline
\end{tabular}

*Only those accident types and gender/job category combinations with at least one occurrence appear in this table. 
Los Alamos National Laboratory 2003

OSHA Data

Appendix V. Number of Workdays Lost or with Restricted Activity in Each Accident Category by Gender and Job Category*

\begin{tabular}{|l|l|r|r|}
\hline \multicolumn{2}{|c|}{} & \multicolumn{2}{|c|}{ Women } \\
& \multicolumn{2}{|c|}{ Job Category } \\
& \multicolumn{2}{|c|}{$\begin{array}{c}\text { Technical } \\
\text { Support }\end{array}$} \\
& $\begin{array}{c}\text { Days } \\
\text { Restricted }\end{array}$ & $\begin{array}{c}\text { Days } \\
\text { Lost }\end{array}$ \\
\hline $\begin{array}{l}\text { Type of } \\
\text { Accident }\end{array}$ & E Codes & & \\
\hline Other Accidents & E916-E928 & & 0 \\
\hline
\end{tabular}

\begin{tabular}{|c|c|c|c|c|c|}
\hline & & \multicolumn{4}{|c|}{ Men } \\
\hline & & \multicolumn{4}{|c|}{ Job Category } \\
\hline & & \multicolumn{2}{|c|}{ Professional } & \multicolumn{2}{|c|}{$\begin{array}{l}\text { Technical } \\
\text { Support }\end{array}$} \\
\hline & & $\begin{array}{c}\text { Days } \\
\text { Restricted }\end{array}$ & $\begin{array}{l}\text { Days } \\
\text { Lost }\end{array}$ & $\begin{array}{c}\text { Days } \\
\text { Restricted }\end{array}$ & $\begin{array}{l}\text { Days } \\
\text { Lost }\end{array}$ \\
\hline Type of Accident & E Codes & \multirow[b]{2}{*}{0} & \multirow[b]{2}{*}{0} & \multirow[b]{2}{*}{0} & \multirow[b]{2}{*}{0} \\
\hline Motor Vehicle Traffic & E810-E819 & & & & \\
\hline Accidental Poisoning - Non-medicinal & E860-E869 & 0 & 0 & 0 & 0 \\
\hline Natural/Environmental Factors & E900-E909 & 0 & 0 & 0 & 0 \\
\hline Submersion/Suffocation/Foreign Bodies & E910-E915 & 0 & 0 & 0 & 0 \\
\hline Other Accidents & E916-E928 & 0 & 0 & 0 & 0 \\
\hline
\end{tabular}

*OSHA events with $>1 \mathrm{E}$ code in the same accident type were counted only once. Only those accident types and gender/job category combinations with at least one occurrence appear in this table. 
Los Alamos National Laboratory 2003

OSHA Data

Appendix W. Age-Adjusted OSHA Illness and Injury Rates by Diagnostic Category

Part 1. Men

\begin{tabular}{|c|c|c|c|c|c|}
\hline Category of Diagnoses & ICD-9-CM Code & $\begin{array}{l}\text { Number of } \\
\text { Diagnoses }\end{array}$ & $\begin{array}{l}\text { Age-Adjusted } \\
\text { Rate per } 1000^{*}\end{array}$ & \begin{tabular}{|c|} 
Lower \\
95\% Confidence \\
Limit per 1000
\end{tabular} & \begin{tabular}{|c|} 
Upper \\
95\% Confidence \\
Limit per 1000
\end{tabular} \\
\hline Infections \& Parasitic Diseases & $001-139$ & 0 & 0 & 0 & 0 \\
\hline Malignant Neoplasms & $140-208,230-234$ & 0 & 0 & 0 & 0 \\
\hline -Digestive Organs & $150-159$ & 0 & 0 & 0 & 0 \\
\hline -Respiratory System & $160-165$ & 0 & 0 & 0 & 0 \\
\hline -Breast & $174-175$ & 0 & 0 & 0 & 0 \\
\hline -Genitourinary & $179-189$ & 0 & 0 & 0 & 0 \\
\hline -Nervous System & $191-192$ & 0 & 0 & 0 & 0 \\
\hline -Leukemia, Lymphoma & $200-208$ & 0 & 0 & 0 & 0 \\
\hline Benign Neoplasms \& Other & $210-229,235-239$ & 0 & 0 & 0 & 0 \\
\hline Endocrine \& Metabolic Diseases & $240-279$ & 0 & 0 & 0 & 0 \\
\hline Blood \& Blood-Forming Organs & $280-289$ & 0 & 0 & 0 & 0 \\
\hline Mental Disorders & $290-319$ & 1 & 0.1 & 0.0 & 0.7 \\
\hline -Alcoholism & 303 & 0 & 0 & 0 & 0 \\
\hline -Drug Abuse & $304-305$ & 0 & 0 & 0 & 0 \\
\hline Nervous System \& Sense Organs & $320-389$ & 0 & 0 & 0 & 0 \\
\hline Circulatory System & $390-459$ & 0 & 0 & 0 & 0 \\
\hline -Hypertension & 401 & 0 & 0 & 0 & 0 \\
\hline -Acute Myocardial Infarction & 410 & 0 & 0 & 0 & 0 \\
\hline -Ischemic Disease, not M.I. & $411-414,429.2$ & 0 & 0 & 0 & 0 \\
\hline -Cerebrovascular Disease & $430-438$ & 0 & 0 & 0 & 0 \\
\hline Respiratory System & $460-519$ & 0 & 0 & 0 & 0 \\
\hline -Upper Respiratory & $460-465,470-478$ & 0 & 0 & 0 & 0 \\
\hline -Pneumonia/Bronchitis & $466,480-487$ & 0 & 0 & 0 & 0 \\
\hline -Chronic Respiratory Conditions & $490-496$ & 0 & 0 & 0 & 0 \\
\hline Digestive System & $520-579$ & 0 & 0 & 0 & 0 \\
\hline -Hernias & $550-553$ & 0 & 0 & 0 & 0 \\
\hline -Gallbladder Disease & $574-575$ & 0 & 0 & 0 & 0 \\
\hline Genitourinary System & $580-629$ & 0 & 0 & 0 & 0 \\
\hline -Benign Prostatic Hypertrophy & 600 & 0 & 0 & 0 & 0 \\
\hline Skin \& Subcutaneous Tissue & $680-709$ & 0 & 0 & 0 & 0 \\
\hline Musculoskeletal System & $710-739$ & 11 & 2.0 & 1.0 & 4.0 \\
\hline -Dorsopathies & $720-724$ & 1 & 0.4 & 0.1 & 2.8 \\
\hline Congenital Anomalies & $740-759$ & 0 & 0 & 0 & 0 \\
\hline Certain Perinatal Conditions & $760-779$ & 0 & 0 & 0 & 0 \\
\hline Symptoms, Signs, \& Ill Defined Cond & $780-799$ & 1 & 0.1 & 0.0 & 0.7 \\
\hline Injury \& Poisoning & $800-999$ & 35 & 5.0 & 3.4 & 7.4 \\
\hline -Fractures, All Sites & $800-829$ & 0 & 0 & 0 & 0 \\
\hline
\end{tabular}

*Standardized to age distribution of 2000 U.S. population.

**Only women aged 18-45 were included in the calculation of the rates for these diagnostic categories. 
Los Alamos National Laboratory 2003

OSHA Data

Appendix W. Age-Adjusted OSHA Illness and Injury Rates by Diagnostic Category

\begin{tabular}{|c|c|c|c|c|c|}
\hline Category of Diagnoses & ICD-9-CM Code & $\begin{array}{l}\text { Number of } \\
\text { Diagnoses }\end{array}$ & $\begin{array}{l}\text { Age-Adjusted } \\
\text { Rate per } 1000 *\end{array}$ & \begin{tabular}{|c|} 
Lower \\
95\% Confidence \\
Limit per 1000
\end{tabular} & \begin{tabular}{|c|} 
Upper \\
95\% Confidence \\
Limit per 1000
\end{tabular} \\
\hline -Dislocations & $830-839$ & 0 & 0 & 0 & 0 \\
\hline -Sprains \& Strains & $840-848$ & 10 & 1.4 & 0.7 & 2.9 \\
\hline -Intracranial Injuries & $850-854$ & 0 & 0 & 0 & 0 \\
\hline -Internal Injuries & $860-869$ & 0 & 0 & 0 & 0 \\
\hline -Open Wounds & $870-897$ & 11 & 1.3 & 0.7 & 2.5 \\
\hline -Other Injuries & $900-999$ & 14 & 2.3 & 1.2 & 4.3 \\
\hline Health Status/Health Serv Contact & V01-V82 & 0 & 0 & 0 & 0 \\
\hline -Family History of Health Problems & V10-V19 & 0 & 0 & 0 & 0 \\
\hline -Circumstances Reproduction/Develop & V20-V28 & 0 & 0 & 0 & 0 \\
\hline -Specific Procedure/Aftercare & V50-V59 & 0 & 0 & 0 & 0 \\
\hline Total & & 48 & 7.2 & 5.2 & 10.1 \\
\hline
\end{tabular}

Part 2. Women

\begin{tabular}{|c|c|c|c|c|c|}
\hline Category of Diagnoses & ICD-9-CM Code & $\begin{array}{l}\text { Number of } \\
\text { Diagnoses }\end{array}$ & $\begin{array}{l}\text { Age-Adjusted } \\
\text { Rate per 1000* }\end{array}$ & \begin{tabular}{|c|} 
Lower \\
95\% Confidence \\
Limit per 1000
\end{tabular} & $\begin{array}{c}\text { Upper } \\
\text { 95\% Confidence } \\
\text { Limit per } 1000\end{array}$ \\
\hline Infections \& Parasitic Diseases & $001-139$ & 0 & 0 & 0 & 0 \\
\hline Malignant Neoplasms & $140-208,230-234$ & 0 & 0 & 0 & $\overline{0}$ \\
\hline -Digestive Organs & $150-159$ & 0 & 0 & 0 & 0 \\
\hline -Respiratory System & $160-165$ & 0 & 0 & 0 & 0 \\
\hline -Breast & $174-175$ & 0 & 0 & 0 & 0 \\
\hline -Genitourinary & $179-189$ & 0 & 0 & 0 & $\overline{0}$ \\
\hline -Nervous System & $191-192$ & 0 & 0 & 0 & 0 \\
\hline -Leukemia, Lymphoma & $200-208$ & 0 & 0 & 0 & 0 \\
\hline Benign Neoplasms \& Other & $210-229,235-239$ & 0 & 0 & 0 & $\overline{0}$ \\
\hline Endocrine \& Metabolic Diseases & $240-279$ & 0 & 0 & 0 & 0 \\
\hline Blood \& Blood-Forming Organs & $280-289$ & 0 & 0 & 0 & 0 \\
\hline Mental Disorders & $290-319$ & 0 & 0 & 0 & $\overline{0}$ \\
\hline -Alcoholism & 303 & 0 & 0 & 0 & 0 \\
\hline -Drug Abuse & $304-305$ & 0 & 0 & 0 & 0 \\
\hline Nervous System \& Sense Organs & $320-389$ & 2 & 0.5 & 0.1 & 1.8 \\
\hline Circulatory System & $390-459$ & 0 & 0 & 0 & 0 \\
\hline -Hypertension & 401 & 0 & 0 & 0 & 0 \\
\hline -Acute Myocardial Infarction & 410 & 0 & 0 & 0 & 0 \\
\hline -Ischemic Disease, not M.I. & $411-414,429.2$ & 0 & 0 & 0 & 0 \\
\hline -Cerebrovascular Disease & $430-438$ & 0 & 0 & 0 & 0 \\
\hline Respiratory System & $460-519$ & 0 & 0 & 0 & 0 \\
\hline -Upper Respiratory & $460-465,470-478$ & 0 & 0 & 0 & 0 \\
\hline
\end{tabular}

*Standardized to age distribution of 2000 U.S. population.

**Only women aged 18-45 were included in the calculation of the rates for these diagnostic categories. 
Los Alamos National Laboratory 2003

OSHA Data

Appendix W. Age-Adjusted OSHA IIlness and Injury Rates by Diagnostic Category

\begin{tabular}{|c|c|c|c|c|c|}
\hline Category of Diagnoses & ICD-9-CM Code & $\begin{array}{l}\text { Number of } \\
\text { Diagnoses }\end{array}$ & $\begin{array}{l}\text { Age-Adjusted } \\
\text { Rate per } 1000 *\end{array}$ & \begin{tabular}{|c|} 
Lower \\
95\% Confidence \\
Limit per 1000
\end{tabular} & \begin{tabular}{|c|} 
Upper \\
95\% Confidence \\
Limit per 1000
\end{tabular} \\
\hline -Pneumonia/Bronchitis & $466,480-487$ & 0 & 0 & 0 & 0 \\
\hline -Chronic Respiratory Conditions & $490-496$ & 0 & 0 & 0 & 0 \\
\hline Digestive System & $520-579$ & 0 & 0 & 0 & 0 \\
\hline -Hernias & $550-553$ & 0 & 0 & 0 & 0 \\
\hline -Gallbladder Disease & $574-575$ & 0 & 0 & 0 & 0 \\
\hline Genitourinary System & $580-629$ & 0 & 0 & 0 & 0 \\
\hline -Endometriosis & 617 & 0 & 0 & 0 & 0 \\
\hline -Ovarian Cysts & $620.0-620.2$ & 0 & 0 & 0 & 0 \\
\hline -Female Genital Pain/Bleeding & $625-626$ & 0 & 0 & 0 & 0 \\
\hline Pregnancy \& Childbirth ${ }^{* *}$ & $630-639$ & 0 & 0 & 0 & 0 \\
\hline Skin \& Subcutaneous Tissue & $680-709$ & 0 & 0 & 0 & 0 \\
\hline Musculoskeletal System & $710-739$ & 15 & 6.3 & 3.3 & 11.8 \\
\hline -Dorsopathies & $720-724$ & 2 & 0.4 & 0.1 & 1.6 \\
\hline Congenital Anomalies & $740-759$ & 0 & 0 & 0 & 0 \\
\hline Certain Perinatal Conditions & $760-779$ & 0 & 0 & 0 & 0 \\
\hline Symptoms, Signs, \& Ill Defined Cond & $780-799$ & 2 & 0.5 & 0.1 & 1.8 \\
\hline Injury \& Poisoning & $800-999$ & 20 & 5.9 & 3.5 & 9.9 \\
\hline -Fractures, All Sites & $800-829$ & 1 & 1.0 & 0.1 & 7.0 \\
\hline -Dislocations & $830-839$ & 0 & 0 & 0 & 0 \\
\hline -Sprains \& Strains & $840-848$ & 15 & 3.5 & 2.1 & 6.1 \\
\hline -Intracranial Injuries & $850-854$ & 0 & 0 & 0 & 0 \\
\hline -Internal Injuries & $860-869$ & 0 & 0 & 0 & 0 \\
\hline -Open Wounds & $870-897$ & 3 & 0.8 & 0.2 & 2.5 \\
\hline -Other Injuries & $900-999$ & 1 & 0.6 & 0.1 & 4.0 \\
\hline Health Status/Health Serv Contact & V01-V82 & 0 & 0 & 0 & 0 \\
\hline -Family History of Health Problems & V10-V19 & 0 & 0 & 0 & 0 \\
\hline -Circumstances Reproduction/Develop & V20-V28 & 0 & 0 & 0 & 0 \\
\hline -Specific Procedure/Aftercare & V50-V59 & 0 & 0 & 0 & 0 \\
\hline Total & & 39 & 13.1 & 8.9 & 19.3 \\
\hline
\end{tabular}

Part 3. Men and Women

\begin{tabular}{|r|r|r|r|r|r|}
\hline Category of Diagnoses & ICD-9-CM Code & $\begin{array}{c}\text { Number of } \\
\text { Diagnoses }\end{array}$ & $\begin{array}{c}\text { Lower } \\
\text { Age-Adjusted } \\
\text { Rate per 1000* }\end{array}$ & $\begin{array}{c}\text { Upper } \\
\text { 95\% Confidence } \\
\text { Limit per 1000 }\end{array}$ & $\begin{array}{c}\text { 95\% Confidence } \\
\text { Limit per 1000 }\end{array}$ \\
\hline Infections \& Parasitic Diseases & $001-139$ & 0 & 0 & 0 & 0 \\
\hline Malignant Neoplasms & $140-208,230-234$ & 0 & 0 & 0 & 0 \\
\hline -Digestive Organs & $150-159$ & 0 & 0 & 0 & 0 \\
\hline -Respiratory System & $160-165$ & 0 & 0 & 0 \\
\hline
\end{tabular}

*Standardized to age distribution of 2000 U.S. population.

**Only women aged 18-45 were included in the calculation of the rates for these diagnostic categories. 
Los Alamos National Laboratory 2003

OSHA Data

Appendix W. Age-Adjusted OSHA Illness and Injury Rates by Diagnostic Category

\begin{tabular}{|c|c|c|c|c|c|}
\hline Category of Diagnoses & ICD-9-CM Code & $\begin{array}{l}\text { Number of } \\
\text { Diagnoses }\end{array}$ & $\begin{array}{l}\text { Age-Adjusted } \\
\text { Rate per } 1000^{*}\end{array}$ & \begin{tabular}{|c|} 
Lower \\
95\% Confidence \\
Limit per 1000
\end{tabular} & \begin{tabular}{|c|} 
Upper \\
95\% Confidence \\
Limit per 1000
\end{tabular} \\
\hline -Breast & $174-175$ & 0 & 0 & 0 & 0 \\
\hline -Genitourinary & 179-189 & 0 & 0 & 0 & 0 \\
\hline -Nervous System & $191-192$ & 0 & 0 & 0 & 0 \\
\hline -Leukemia, Lymphoma & $200-208$ & 0 & 0 & 0 & 0 \\
\hline Benign Neoplasms \& Other & $210-229,235-239$ & 0 & 0 & 0 & 0 \\
\hline Endocrine \& Metabolic Diseases & $240-279$ & 0 & 0 & 0 & 0 \\
\hline Blood \& Blood-Forming Organs & $280-289$ & 0 & 0 & 0 & 0 \\
\hline Mental Disorders & $290-319$ & 1 & 0.1 & 0.0 & 0.5 \\
\hline -Alcoholism & 303 & 0 & 0 & 0 & 0 \\
\hline -Drug Abuse & 304-305 & 0 & 0 & 0 & 0 \\
\hline Nervous System \& Sense Organs & $320-389$ & 2 & 0.1 & 0.0 & 0.6 \\
\hline Circulatory System & $390-459$ & 0 & 0 & 0 & 0 \\
\hline -Hypertension & 401 & 0 & 0 & 0 & 0 \\
\hline -Acute Myocardial Infarction & 410 & 0 & 0 & 0 & 0 \\
\hline -Ischemic Disease, not M.I. & $411-414,429.2$ & 0 & 0 & 0 & 0 \\
\hline -Cerebrovascular Disease & $430-438$ & 0 & 0 & 0 & 0 \\
\hline Respiratory System & $460-519$ & 0 & 0 & 0 & 0 \\
\hline -Upper Respiratory & $460-465,470-478$ & 0 & 0 & 0 & 0 \\
\hline -Pneumonia/Bronchitis & $466,480-487$ & 0 & 0 & 0 & 0 \\
\hline -Chronic Respiratory Conditions & $490-496$ & 0 & 0 & 0 & 0 \\
\hline Digestive System & $520-579$ & 0 & 0 & 0 & 0 \\
\hline -Hernias & $550-553$ & 0 & 0 & 0 & 0 \\
\hline -Gallbladder Disease & $574-575$ & 0 & 0 & 0 & 0 \\
\hline Genitourinary System & $580-629$ & 0 & 0 & 0 & 0 \\
\hline -Benign Prostatic Hypertrophy & 600 & 0 & 0 & 0 & 0 \\
\hline -Endometriosis & 617 & 0 & 0 & 0 & 0 \\
\hline -Ovarian Cysts & $620.0-620.2$ & 0 & 0 & 0 & 0 \\
\hline -Female Genital Pain/Bleeding & $625-626$ & 0 & 0 & 0 & 0 \\
\hline Pregnancy \& Childbirth** & $630-639$ & 0 & 0 & 0 & 0 \\
\hline Skin \& Subcutaneous Tissue & $680-709$ & 0 & 0 & 0 & 0 \\
\hline Musculoskeletal System & $710-739$ & 26 & 3.1 & 2.0 & 4.7 \\
\hline -Dorsopathies & $720-724$ & 3 & 0.4 & 0.1 & 1.4 \\
\hline Congenital Anomalies & $740-759$ & 0 & 0 & 0 & 0 \\
\hline Certain Perinatal Conditions & $760-779$ & 0 & 0 & 0 & 0 \\
\hline Symptoms, Signs, \& Ill Defined Cond & $780-799$ & 3 & 0.2 & 0.1 & 0.6 \\
\hline Injury \& Poisoning & $800-999$ & 55 & 5.3 & 3.9 & 7.1 \\
\hline -Fractures, All Sites & $800-829$ & 1 & 0.2 & 0.0 & 1.4 \\
\hline -Dislocations & $830-839$ & 0 & 0 & 0 & 0 \\
\hline
\end{tabular}

*Standardized to age distribution of 2000 U.S. population.

**Only women aged 18-45 were included in the calculation of the rates for these diagnostic categories. 
Los Alamos National Laboratory 2003

OSHA Data

Appendix W. Age-Adjusted OSHA Illness and Injury Rates by Diagnostic Category

\begin{tabular}{|c|c|c|c|c|c|}
\hline Category of Diagnoses & ICD-9-CM Code & $\begin{array}{l}\text { Number of } \\
\text { Diagnoses }\end{array}$ & $\begin{array}{l}\text { Age-Adjusted } \\
\text { Rate per } 1000 *\end{array}$ & $\begin{array}{c}\text { Lower } \\
\text { 95\% Confidence } \\
\text { Limit per } 1000\end{array}$ & $\begin{array}{c}\text { Upper } \\
\text { 95\% Confidence } \\
\text { Limit per } 1000\end{array}$ \\
\hline -Sprains \& Strains & $840-848$ & 25 & 2.1 & 1.3 & 3.3 \\
\hline -Intracranial Injuries & $850-854$ & 0 & 0 & 0 & 0 \\
\hline -Internal Injuries & $860-869$ & 0 & 0 & 0 & 0 \\
\hline -Open Wounds & $870-897$ & 14 & 1.2 & 0.7 & 2.1 \\
\hline -Other Injuries & $900-999$ & 15 & 1.8 & 1.0 & 3.1 \\
\hline Health Status/Health Serv Contact & V01-V82 & 0 & 0 & 0 & 0 \\
\hline -Family History of Health Problems & V10-V19 & 0 & 0 & 0 & 0 \\
\hline -Circumstances Reproduction/Develop & V20-V28 & 0 & 0 & 0 & 0 \\
\hline -Specific Procedure/Aftercare & V50-V59 & 0 & 0 & 0 & 0 \\
\hline Total & & 87 & 8.7 & 6.9 & 11.1 \\
\hline
\end{tabular}

*Standardized to age distribution of 2000 U.S. population.

**Only women aged 18-45 were included in the calculation of the rates for these diagnostic categories. 